\title{
Novel Quantum Phases in Low Dimensions
}

\author{
Zhao Zhang \\ Tianjin, China
}

BS, University of Science and Technology of China, 2012

A Dissertation presented to the Graduate Faculty of the University of Virginia in Candidacy for the Degree of Doctor of Philosophy

Department of Physics

University of Virginia

May, 2017 
(C) Zhao Zhang 2017

All Rights Reserved 


\section{Acknowledgements}

The research presented in this dissertation could not have been done without the supervision of Israel Klich and Jeffrey Teo. I am lucky to have both of them as my advisors.

I remember during my first conversation with Israel, he introduced me to amplituhedron, a newly proposed alternative to quantum field theory, to point out the fallacy in my reasoning behind switching from high energy phenomenology to condensed matter theory, worrying that the former already has a well-developed framework and nothing original is left to be done. This exemplifies not only his consistently high expectation of student, but also his completely optimistic attitude towards research, both of which are key to the completion of my thesis. Yet, what I feel most lucky about is that he genuinely cares about my research interests and always goes out of his way to accommodate them.

Upon his arrival, Jeffrey diagnosed my inefficient bottom-up approach to research

and helped me transform to a top-down method and get on track as quickly as possible, which he did with incredible patience. I'm grateful for the various learning opportunities he provided to attend conferences and give presentations. Despite being a young professor, he is a walking encyclopedia of his research area, and it's just reassuring to know that any question I have, I can have it answered right here in his office. My learning from Jeffrey goes beyond physics, as he also sets a good example of being a great scholar and a great person. 
I thank professor Paul Fendley for showing me the beauty of statistical mechanics. Attending his insight-filled lectures has been for high of my week for two semesters, and to date reading his crystal clear articles is still my not-so-guilty pleasure. Other professors that have offered guidance and support at different stages of my $\mathrm{PhD}$ program include, but are not limited to, Diana Vaman, Seunghun Lee, and Mark Williams. I especially treasure the time I spent at professor William's lab, where I got to work closely with senior students Zongyi Gong, Tushita Patel and Anoosha Reddy, who couldn't be more knowledgable or pleasant to learn from. I thank Gabriel Wong particularly for encouraging me to work on a project with Israel and generally for sharing many useful discussions. Finally, I thank Rafael Alexander for lessons on measurement based quantum computation and related topics. 


\section{Table of Contents}

Acknowledgements ................... . . ii

List of Figures . . . . . . . . . . . . . . . . . . . vi

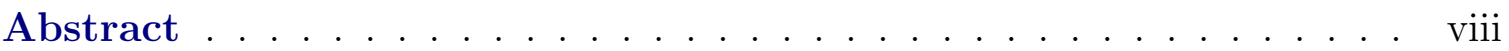

\section{Chapter}

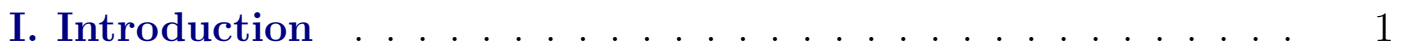

1.1 Scaling of entanglement entropy . . . . . . . . . . . . . 1

1.1.1 Area law and its violation . . . . . . . . . 2

1.1.2 (Colored) Motzkin and Fredkin spin chain . . . . . 3

1.2 Classification of topological phases . . . . . . . . . . 8

1.2.1 $\mathbb{Z}$ classification of time-reversal symmetric topological superconductor . . . . . . . . . . . . . 9

1.2.2 Collapse of $\mathbb{Z}$ classification under interaction . . . . 12

1.2 .3 Intrinsic topological order . . . . . . . . . . . 13

II. Deformed Motzkin Spin Chain . . . . . . . . . . . . . 16

2.1 A Frustration-Free Deformation . . . . . . . . . . . . . . 17

2.2 The Colorful Model . . . . . . . . . . . . . . . . . . 21

2.3 Entanglement Entropy . . . . . . . . . . . . . . 23

2.3.1 Extensive entropy for $t>1, s>1 \ldots 26$

2.3.2 Bounded entropy for $t<1$........... . . 28

2.4 A novel quantum phase transition . . . . . . . . . . . 32

III. Deformed Fredkin Spin Chain . . . . . . . . . . . . . . 33

3.1 Multi-parameter Hamiltonian and ground state . . . . . . . . 33

3.2 Entanglement entropy . . . . . . . . . . . . . . . 37

3.2.1 $t>1, s>1$ phase: Volume scaling of entropy. . . . . 39

3.2.2 $t<1$ and any $s$ : Bounded entanglement entropy. . . 43 
3.3 Scaling of the Spectral Gap . . . . . . . . . . . . . . . 46

3.3.1 Super-exponential Upper bound in the $t>1, s=1$ Phase . . . . . . . . . . . . . 46

3.3.2 Exponential Upper Bound in the $t>1, s=1$ Phase 49

IV. Coupled Wire Model of Majorana Surfaces of Topological Superconductors . . . . . . . . . . . . . . . . 52

4.1 Coupled wire construction of surface Majorana cones . . . . . 53

4.1.1 The $s o(N)_{1}$ current algebra . . . . . . . . . . . 57

4.1.2 Bosonizing even Majorana cones . . . . . . . . . . 60

4.2 Gapping surface Majorana cones . . . . . . . . . . . . . 63

4.2.1 Gapping even Majorana cones . . . . . . . . . . . . 67

4.2 .2 Gapping odd Majorana cones . . . . . . . . . . . . . 78

4.2.3 Gapping by fractional quantum Hall stripes . . . . . 91

4.3 Surface topological order . . . . . . . . . . . . . . . . 93

4.3.1 Summary of anyon contents . . . . . . . . . . . 97

4.3.2 The 32-fold tensor product structure . . . . . . . 100

4.4 Other possibilities . . . . . . . . . . . . . . 111

4.4.1 Consequence of the emergent $E_{8}$ when $N=16 \ldots 112$

4.4.2 Alternative conformal embeddings . . . . . . . . 115

V. Conclusions and Outlooks . . . . . . . . . . . . . 117

Appendicies . . . . . . . . . . . . . . . . . . . . 120 


\section{List of Figures}

\section{Figure}

1.1 A spin-1 configuration, and its representation as a set of parentheses and a Motzkin path. . . . . . . . . . . . . . . .

1.2 Coloring a Motzkin path leads to a higher spin configuration. Representations of a spin-2 state as a a set of parentheses and as Motzkin path, where $1,-1 \leftrightarrow(,) \leftrightarrow$ red and $2,-2 \leftrightarrow[,] \leftrightarrow$ blue. . . . . . . .

2.1 Motzkin paths require color correlations between the color of an up step and the color of the first step going down at the same height. The Motzkin path reaching height $m=5$ in the middle (left panel) contains more color correlations between the halves of the chain than a path of height $m=0$ (right panel). Favoring higher paths leads to more entanglement. . . . . . . . . . . . . . . . . .

2.2 Iterative procedure to flatten a mountain to the ground, where steps 2 and 3 can be interchanged. . . . . . . . . . . . . . .

2.3 Two different sequences of moves to relate local state $|000\rangle$ to $|l 0 r\rangle$, and the relative weights of each state involved. . . . . . . . . .

2.4 Different sequences of $L, R, F$ moves to get a hill (solid) from a starting plateau (dash dot) with intermediate plateaus after each move (dash). (a) Two different sequences of moves $R_{h-1} L_{h+1} L_{h+2} L_{h+3} R_{h-2} L_{h+4} L_{h+5}$ and $L_{h+1} L_{h+2} R_{h-1} L_{h+3} L_{h+4} L_{h+5} R_{h-2}$ with the same location of hill always give the same relative weight. (b) A sequence of moves with hill location different from those in (a) could generically give a different relative weight, except when relation $(2.2)$ is satisfied. . . . . . . . . . . . . . 20

2.5 A caricature of the ground state of our model. . . . . . . . . . . . 23

2.6 Three representative paths of different heights at position $k$ to generate a path that ends with height $m$ at position $k+1$. The increments in the area below the paths are the areas sandwiched between two vertical lines, which are $m+\frac{1}{2}, m, m-\frac{1}{2}$ respectively for red, green and blue paths. . . . . . . . . . . . . . . . . .

2.7 Entanglement entropy of the first $n$ sites in a chain of $2 n$ sites for various phases of the colored and uncolored area-weighted Motzkin state. . . . . . . . . . . . . . . . . . 
3.1 Different ways of "flattening" a hill must have the same amplitude. Note that the colors have been interchanged during the procedure, this, however, is not an obstruction as all colorings appear with the same amplitude. . . . . . . . . . . . . . . . . . . .

3.2 A spin configuration corresponding to a colored Dyck path and the corresponding height function $h(l)$. . . . . . . . . . . . .

3.3 The "flattening" of a hill and it's amplitude. Starting from the left, we reduce the first by using the Fredkin move $|\uparrow \uparrow \downarrow\rangle \longrightarrow t_{A}|\uparrow \downarrow \uparrow\rangle$. This process is repeated for each peak until the lowest height hill is achieved. . . . . . . . . . . . . . . . .

3.4 An representative walk in the superposition of $|\xi\rangle$ that crosses the threshold of the cut-off when acted on by the operator $\left(t \mid \downarrow_{n-1}^{r} \uparrow_{n}^{b} \downarrow_{n+1}^{b}\right.$ \rangle$\left.-\left|\uparrow_{n-1}^{b} \downarrow_{n}^{b} \downarrow_{n+1}^{r}\right\rangle\right)\left(\left\langle\downarrow_{n-1}^{r} \uparrow_{n}^{b} \downarrow_{n+1}^{b}\right| t-\left\langle\uparrow_{n-1}^{b} \downarrow_{n}^{b} \downarrow_{n+1}^{r}\right|\right) \ldots . . . \ldots .$.

3.5 Two representative walks in $Q_{-2}$. The light blue one can be shifted in the direction of the orange arrow to become the dark blue one with a relative weight increase of $t^{n-1}$ corresponding to the area of the shaded regions. . . . . . . . . . . . . . . . .

4.1 (Left) Coupled wire model (4.3) of $N$ gapless surface Majorana cones. (Right) Fractionalization (4.33) and coupled wires construction (4.37) of gapped anomalous and topological surface state. . . . . . . . . 53

4.2 The energy spectrum of the coupled Majorana wire model (4.3) . . 54

4.3 Coupled Majorana wire model on the surface of (a) a stack of alternating $p_{x} \pm i p_{y}$ superconductors, and (b) a class DIII topological superconductor (TSC) with alternating TR breaking surface domains. (c) A dislocation. . . . . . . . . . . . . . . . . . . . .

4.4 Energy spectrum of the $N=2$ coupled Majorana wire model with inter-flavor mixing. . . . . . . . . . . . . . .

4.5 Interwire gapping terms (4.37) (green rectangular boxes) between chiral fractional $\mathcal{G}_{N}^{R, \pm}, \mathcal{G}_{N}^{L, \pm}$ sectors (resp. $\otimes, \odot$ ) in opposite direction.

4.6 Gapping $N$ surface Majorana cones by inserting $(2+1) \mathrm{D} G_{N}$ stripe state and removing edge modes by current-current backscattering interaction. . . . . . . . . . . . . . . . .

4.7 Chiral interface (highlighted line) between a time reversal breaking gapped region and a TR symmetric topologically ordered gapped region. 94

4.8 The $G_{N}$ topological order of a quasi-2D slab with time reversal symmetric gapped top surface and time reversal breaking gapped bottom surface . . . . . . . . . . . . . . . . . . 


\section{Abstract \\ Novel Quantum Phases in Low Dimensions \\ by Zhao Zhang}

Advisor: Israel Klich and Jeffrey C.Y. Teo

We introduce a continuous family of frustration-free Hamiltonians with exactly solvable ground states. We prove that the ground state of our model is non-degenerate and exhibits a novel quantum phase transition from bounded entanglement entropy to a massively entangled state with volume entropy scaling. The ground state may be interpreted as a deformation away from the uniform superposition of colored Motzkin paths, shown by Movassagh and Shor [80], that has a large (square-root) but subextensive scaling of entanglement. We carry out the same procedure for both integer and half integer versions of the spin chain, and established upper bounds on the spectral gap for certain phases of the model.

Time reversal symmetric topological superconductors in three spatial dimensions carry gapless surface Majorana fermions. They are robust against any time reversal symmetric single-body perturbation weaker than the bulk energy gap. We mimic the massless surface Majorana's by coupled wire models in two spatial dimensions. We introduce explicit many-body inter-wire interactions that preserve time reversal symmetry and give energy gaps to all low energy degrees of freedom. We show the gapped models generically carry non-trivial topological order and support anyonic excitations. 


\section{Chapter I}

\section{Introduction}

\subsection{Scaling of entanglement entropy}

Entanglement is a quantum mechanical phenomenon that occurs when particles are prepared or interact in such a way that the state of each particle cannot be described independently of the others, even when they are separated far apart. This 'spooky' action at a distance, or non-locality, is the reason that quantum mechanics suffered from severe theoretical skepticism since its early days. At the same time, entanglement is also responsible for the major speed-up in quantum computation algorithms compared to its classical counterpart, and certain quantum communication protocols, such as teleportation, relies exclusively on entangled states[6].

Thermodynamic entropy in classical statistical mechanics quantifies the uncertainty in identifying the exact microscopic configuration of a thermodynamic system in a macroscopically specified state. In quantum mechanics, on the other hand, the notion of randomness is inherent, and one of the main source is entanglement. Consider a quantum many-body system with pure non-degenerate ground state $|\psi\rangle$. At zero temperature, it has vanishing von Neumann entropy

$$
S(\rho)=-\operatorname{Tr}(\rho \ln \rho)
$$


where $\rho=|\psi\rangle\langle\psi|$ is its density matrix. Now, we divide the system into subregions $A$ and $B$, the reduced density matrix $\rho_{A}=\operatorname{Tr}_{B} \rho$ will generally be a mixed state, which has non-vanishing von Neumann entropy $S\left(\rho_{A}\right)$. This entropy of a subsystem is defined as entanglement entropy. The main motivations for studying the scaling of entanglement entropy stemmed from its relation to black hole entropy and holographic principle, distribution of quantum correlations, numerical simulation, and detecting topological order of many-body systems[27, 66].

\subsubsection{Area law and its violation}

In the 1970s, Bekenstein and Hawking set out to study thermodynamic entropy in black holes. Bekenstein showed that the thermodynamic entropy of a black hole scales as its area. But it wasn't until Bombelli in 1986 [13] that entanglement entropy of the ground state of a free massless field is shown to be proportional to the area of its boundary. Since then, scaling with the system's size became of fundamental importance in studying entanglement entropy in many-body systems. At first glance, it might be tempting to assume that entanglement entropy should have the same scaling behavior as that of the thermodynamic entropy, which is an extensive quantity, which scales with volume. Yet, volume scaling turns out to be highly unusual, and the entanglement entropy of a ground state typically obeys an area law, or at most one with a logarithmic correction, despite the fact that it has been shown that the average entanglement entropy of a random quantum state satisfies a volume law [91, 35, 110].

The reason why area law is common is that interactions between particles in realistic Hamiltonians tend to be local, e.g. between nearest and next nearest neighbors. As a result, low energy states are heavily constrained by locality and only occupy a small corner in a huge many-body Hilbert space, where a random state will most likely exhibit a volume-scaling entanglement entropy. In other words, some states in the Hilbert space are more relevant than others. So for gapped, local Hamiltonians, it 
is suspected that only states with area law scaling qualify as low energy state candidates. Moreover, one can show that starting from these states, only a small manifold of states in the full Hilbert space can be reached by evolving under a local Hamiltonian over a polynomial time of system size[92]. This makes the Hilbert space little more than a convenient illusion, and we could target the corner of relevant states directly with a convenient class of states such as tensor network states. Since the renormalization group $(\mathrm{RG})$ is designed to tell the relevant degrees of freedom from the irrelevant, it is natural to use RG method on tensor network states[90].

Most known results about area law are in 1-dimensional (1D) systems, where it means the entanglement entropy is bounded from above by a constant independent of the system size. In particular, a rigorous theorem on 1D gapped, local spin models was established by Hastings[48], where locality enters the proof by the existence of Lieb-Robinson bounds, which is also essential in the proof of exponetial decay of correlation functions in these models.

While the area law has been shown to apply to a wide variety of gapped systems, its violations in the ground state of gapless systems have also been illustrated in several systems. For example, in (1+1)-dimensional conformal field theories, entanglement entropy has been shown to have logarithmic scaling with the system size[49, 17, 53]. In addition, Fermi liquids also exhibit a logarithmic violation of area law in any dimension $[142,39]$. Recently, more examples have been found that show more severe violations of the area law in one-dimensional spin systems[51, 42, 125, 96]. However,

these exotic scaling behavior are achieved at the expense of either introducing a local Hilbert space with very large dimension or sacrificing translational invariance.

\subsection{2 (Colored) Motzkin and Fredkin spin chain}

In 2012, Bravyi et al. proposed a toy model of spin chain with a frustration free (FF) Hamiltonian that describes criticality[15]. A frustration free Hamiltonian is a 


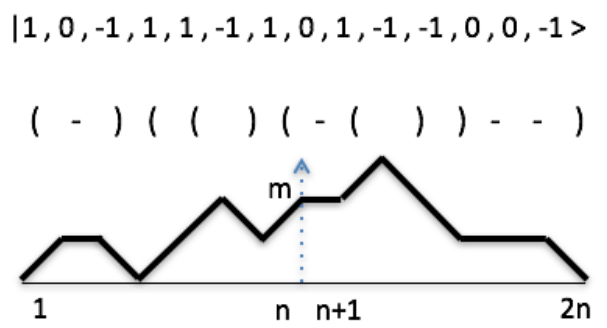

Figure 1.1: A spin-1 configuration, and its representation as a set of parentheses and a Motzkin path.

Hamiltonian $H=\sum_{u \in \Lambda} Q_{u}$, where $\Lambda$ is the lattice, such that its ground state is the common lowest-energy eigenstate of all operator $Q_{u}$ 's. The ground state of their Hamiltonian, the 'Motzkin state', derived its name from the classical combinatorial problem of counting the number of Motzkin paths of a given length.

Definition I.1. A Motzkin walk (or path) on $2 n$ steps is any path from $(0,0)$ to $(0$, 2n) with steps (1, 0), (1, 1) and (1,-1) that never passes below the x-axis.

Pictorially, a Motzkin walk corresponds to a mountain range that is located between site 0 and $2 n$. A Motzkin walk naturally encodes a spin-1 state $\left|\sigma_{1}, \ldots \sigma_{2 n}\right\rangle$ constructed by assigning for the local spin variables $\sigma_{k}=+1,-1$ or 0 if the walk goes up, down, or stays flat at site $k$. As depicted in Fig. 1.1, Motzkin paths can be thought of as grammatically allowed choices of arranging left and right parentheses in a sentence where a right parenthesis is only permitted to be placed if there is an unpaired left parenthesis to its left. Following the notation of [15], the local spin basis $|l\rangle,|r\rangle$ and $|0\rangle$ now corresponding to the $S^{z}=+1,-1$, and 0 states respectively.

The ground state is a uniform superposition of all Motzkin paths is given by

$$
|G S\rangle=\frac{1}{\mathcal{N}} \sum_{w \in\{\text { Motzkin walks }\}}|w\rangle,
$$

where $|w\rangle$ is a Motzkin walk of size $n$ and $\mathcal{N}$ is the normalization factor. The Motzkin state is the unique ground state of the frustration-free Hamiltonian imposing only 
local constraints,

$$
H=|r\rangle\left\langle\left. r\right|_{1}+\mid l\right\rangle\left\langle\left. l\right|_{2 n}+\sum_{j=1}^{2 n-1} \pi_{j, j+1},\right.
$$

where projectors acting on spin $j, j+1$

$$
\pi_{j, j+1}=|\phi\rangle\left\langle\left.\phi\right|_{j, j+1}+\mid \psi\right\rangle\left\langle\left.\psi\right|_{j, j+1}+\mid \theta\right\rangle\left\langle\left.\theta\right|_{j, j+1},\right.
$$

with

$$
|\phi\rangle=\frac{1}{\sqrt{2}}(|0 l\rangle-|l 0\rangle),|\psi\rangle=\frac{1}{\sqrt{2}}(|0 r\rangle-|r 0\rangle),|\theta\rangle=\frac{1}{\sqrt{2}}(|00\rangle-|l r\rangle) .
$$

The authors in ref. [15] showed that the entropy of half of a Motzkin chain with size $\mathrm{n}$

$$
S=\frac{1}{2} \log n+c_{n}
$$

where $c_{n}$ goes to a constant as $n \rightarrow \infty$, conjecturing that a power-law scaling of $S$ can be achieved by introducing two types of brackets. They also gave an upper bound on the spectral gap of $O\left(n^{-\frac{1}{2}}\right)$ using variational wave function and a lower bound of $O\left(n^{-O(1)}\right)$ using more complicated mathematical machinery.

In this context, it is important to point out that, as shown in [79], there are three distinct regimes for Hamiltonians of 1D spin chains, with a local Hilbert space of dimension $d$, whose terms are generic local projectors of fixed rank $r$ : (i) When $r>d^{2} / 4$, the Hamiltonian is frustrated for sufficiently large spin chains and analytical as well as numerical work showed that no zero-energy ground states exist, (ii) a regime where $d \leq r \leq d^{2} / 4$ where many zero-energy ground states are allowed analytically and where numerical investigation suggests that they all carry a large amount of entanglement, and (iii), a frustration-free regime with $r<d$ where the ground states can be represented by a matrix product state. The Motzkin path-based models first 


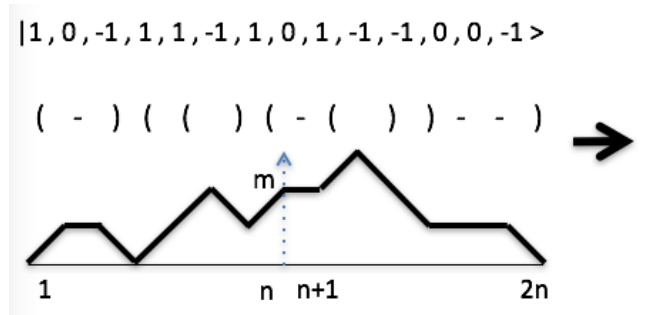

$|1,0,-1,2,1,-1,1,0,2,-2,-1,0,0,-1\rangle$

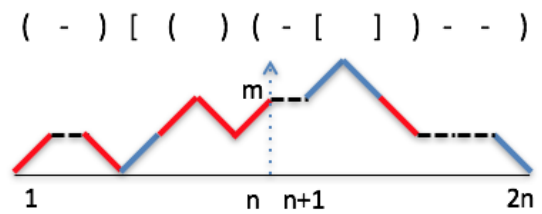

Figure 1.2: Coloring a Motzkin path leads to a higher spin configuration. Representations of a spin-2 state as a a set of parentheses and as Motzkin path, where $1,-1 \leftrightarrow(,) \leftrightarrow$ red and $2,-2 \leftrightarrow[,] \leftrightarrow$ blue.

introduced in [15] and later with the addition of color in [80] represent a particular case where the Hamiltonian turned out to be frustration-free for $r=d=3$. For this reason, the authors in [15] have pointed out that any arbitrarily small deformations of the projectors in the Motzkin path Hamiltonian will make it generic and thus throw its ground state into the frustrated regime. In the model presented in this paper, however, we derive a simple equation that relates the weights of local moves at different sites of the chain and thus deform the local projectors away from the uniform case in such a way that frustration-freeness of the Hamiltonian is maintained.

Movassagh and Shor later extended the model by Bravyi et al. to a colored version of the Motzkin spin chain[80]. They labeled different types of parentheses by adding a color degree of freedom and restricting their order by adding local projectors in the Hamiltonian to enforce matching of color between neighboring pairs. In an $s$-colored model, where $s$ is the number of colors, the local Hilbert space is $(2 s+1)$-dimensional and spanned by the basis states $|0\rangle,\left|l^{1}\right\rangle, . .\left|l^{s}\right\rangle,\left|r^{1}\right\rangle, . .\left|r^{s}\right\rangle$. Fig. 1.2 depicts a coloring choice for the Motzkin path in Fig. 1.1 and the associated spin state.

The ground state of the colored Motzkin Hamiltonian is a uniform superposition of colored Motzkin paths of length $2 n$. The entropy of a half chain was shown to scale as $\log s \sqrt{n}$ in the thermodynamic limit. The reason behind the $\sqrt{n}$ scaling stems from the fact that the average displacement after $n$ steps of a random walk, represented in the colored Motzkin model by the number of unmatched colors, scales as $\sqrt{n}$. 
What Movassagh and Shor failed to realize, however, is that once the color degree of freedom is added, the uniform superposition in the ground state does not achieve maximal scaling of entanglement entropy any longer. The possibility that one can achieve this by enhancing higher mountains in the superposition without sacrificing frustration freeness at all is, of course, not obvious. In chapter II, I will show rigorously that it is indeed possible and it turns out and any slight frustration-free deviation from the Motzkin spin model will change the behavior of its entanglement entropy and spectral gap dramatically[146].

As is well known for the Heisenberg model, integer and half-integer spin chains can behave very differently. So it's worth studying a spin- $\frac{1}{2}$ version of the Motzkin models. In fact, corresponding combinatorics problem called Catalan number for configurations of spin- $\frac{1}{2}$ chain, Dyck paths, already exists in math literature.

Definition I.2. A Dyck walk (or path) on $2 n$ steps is any path from $(0,0)$ to $(0,2 n)$ with steps $(1,1)$ and $(1,-1)$ that never passes below the $x$-axis.

A Dyck walk can be mapped to a spin configuration of a spin- $1 / 2$ chain $\left|\sigma_{1} \sigma_{2} \ldots \sigma_{2 n}\right\rangle$ with $\sigma_{k}=+1 / 2$ or $-1 / 2$ for a $(1,1)$ or $(1,-1) k$ th step respectively. When each step is assigned a color $c_{k}$ from a palette of $s$ colors, $\left|\uparrow_{k}^{c}\right\rangle\left(\left|\downarrow_{k}^{c}\right\rangle\right)$ corresponds to a state $\sigma_{k}=c_{k} / 2\left(\right.$ resp. $\left.\sigma_{k}=-c_{k} / 2\right)$. And a colored Dyck walk can, therefore, represent the spin configuration of any half-integer spin chain. To design an Hamiltonian whose ground state is a uniform superposition of Dyck paths, one just need to replace projectors in the bulk in Hamiltonian 1.3 with those that swap a pair of up-down spins with a single neighboring spin,

$$
H_{j}=|\uparrow\rangle_{j}\langle\uparrow|\otimes| S\rangle_{j+1, j+2}\langle S|+| S\rangle_{j, j+1}\langle S|\otimes| \downarrow\rangle_{j+2}\langle\downarrow|
$$

where $\left|S_{i, j}\right\rangle$ is the singlet state $\frac{1}{\sqrt{2}}\left(\left|\uparrow_{i}\right\rangle\left|\downarrow_{j}\right\rangle-\left|\downarrow_{i}\right\rangle\left|\downarrow_{j}\right\rangle\right) .[24,104]$ Due to the relation of this operator to Fredkin (controlled-swap) gate, the model is named Fredkin spin 
chain. In Ref.[104], the authors established similar results on Fredkin spin chain in parallel with those for Motzkin and colored Motzkin chain. In chapter III, we will improve these results and generalize the model discussed in chapter II[105, 147].

\subsection{Classification of topological phases}

Another crucial ingredient of quantum mechanics, besides entanglement, is the phase factor of wave function. Until the discovery of Aharonov-Bohm effect and Berry phase, phase factors in quantum mechanics are merely perceived as an auxiliary degree of freedom to facilitate interference and gauge invariance, as the amplitude of a wave function appeared to be the only physical observable. Berry phase, on the other hand, is the gauge invariant phase difference acquired when a quantum system undergoes an adiabatic evolution in a closed loop in the parameter space of its Hamiltonian. Because it can be expressed as the integral of local geometrical quantity in the parameter space, i.e. Berry curvature, it is also called geometrical phase. Berry phase has a variety of implications in a wide range of areas in physics. In the context of condensed matter physics, Bloch bands provided a natural platform for its manifestation as it captures the topological structure of the space of Bloch Hamiltonians[143].

A timeless theme of condensed matter physics is to understand how large numbers of particles organize themselves under different conditions and form distinctive phases of matter. Classical phase transitions result from thermal fluctuations, and the nonanalyticity of free energy. As temperature varies, energy competes with entropy to decided whether ordered or disordered configuration wins. Quantum phase transitions, however, happens at zero temperature, and are driven exclusively by quantum fluctuations of the ground state, whose energy is a non-analytic function of some coefficient of the Hamiltonian in thermodynamic limit. As the coefficient approaches a critical value, the ground state changes dramatically. For a long time, our under- 
standing of phase transition has been limited to the Landau-Ginzburg paradigm of spontaneous symmetry breaking, where phases of matter are classified by their underlying symmetry. In the past few decades, following the discovery of integer quantum Hall effect and topological insulators (TIs)[47], it became clear that the classification by symmetry alone is incomplete and one can have topologically distinct phases characterized by topological invariants within the same symmetry class. These invariants take integer values and are protected by symmetries against adiabatic deformations as long as bulk gap remains open, i.e. without a quantum phase transition that involves gap closing and reopening. This kind of topological phases are called symmetry protected topological (SPT) phases.

\subsection{1 $\mathbb{Z}$ classification of time-reversal symmetric topological superconduc- tor}

The simplest SPT phases are those of fully gapped free fermionic systems called topological band insulators (TBIs) including TIs and topological superconductors (TSCs). The Altland-Zirnbauer's ten-fold way provide a complete classification of these systems described by bilinear Bloch-BdG Hamiltonians[5]. In this scheme, topological invariants are protected by three non-spatial global symmetries, time-reversal (TR), particle-hole $(\mathrm{PH})$, and chiral. TR and $\mathrm{PH}$ symmetry can both square to +1 or -1 , or be absent. In addition, their product, chiral symmetry can still be present when neither of them is, and always square to +1 . So the ten-fold way exhausts all possible symmetry classes in the ensemble of random Hamiltonians. Within each symmetry class, topologically distinct phases are classified by different bulk topological invariants in different spatial dimensions such as Chern number or winding number, as well as number of gapless boundary modes[22].

We now focus on the $\mathbb{Z}$ classification of non-interacting three dimensional class DIII topological superconductors (TSCs) [109, 62, 93]. Candidate materials for this 
class include superfluid ${ }^{3} \mathrm{He}-\mathrm{B}[127,7,23,83]$ and copper dopped Bismuth Selinide $\mathrm{Cu}_{x} \mathrm{Bi}_{2} \mathrm{Se}_{3}[37,107]$. Hamiltonians in this class has $\mathrm{TR}$ symmetry $T$ with $T^{2}=-1$, PH symmetry $C$ with $C^{2}=1$, and chiral symmetry $\Pi$ with $\Pi^{2}=1$. In momentum space, it has Bogoliubov-de Gennes (BdG) Hamiltonian in Nambu basis $\xi(\mathbf{k})=\left(c_{\uparrow}(\mathbf{k}), c_{\downarrow}(\mathbf{k}), c_{\downarrow}^{\dagger}(-\mathbf{k})^{T},-c_{\uparrow}^{\dagger}(-\mathbf{k})\right)$.

$$
H=\int d \mathbf{k} \xi^{\dagger}(\mathbf{k}) H_{B d G}(\mathbf{k}) \xi(\mathbf{k})
$$

By construction, the artificial Nambu doubling requires the matrix $H_{B d G}$ to satisfy PH symmetry $C H_{B d G}(\mathbf{k}) C^{-1}=-H_{B d G}(-\mathbf{k})$, where the antiunitary PH operator $C=\sigma_{y} \tau_{y} \mathcal{K}$, where $\sigma_{y}$ acts on spin and $\tau_{y}$ acts on Nambu degrees of freedom. Physically, this means that excitations at $\mathbf{k}$ in the negative energy bands are actually holes of particles at $\mathbf{- k}$ in positive ones. In much the same way as quantum spin Hall effect can be thought of as two copies of TR breaking quantum Hall, the two copies of spinless (or spin-polarized) fermions form TR partners so that $H_{B d G}$ is TR symmetric $T H_{B d G}(\mathbf{k}) T^{-1}=H_{B d G}(-\mathbf{k})$, where the antiunitary $\mathrm{TR}$ operator $T=i \sigma_{y} \mathcal{K}$. The product of $\mathrm{PH}$ and $\mathrm{TR}$ gives a chiral symmetry that anticommutes with the Hamiltonian $\Pi H_{B d G}(\mathbf{k}) \Pi=-H_{B d G}(\mathbf{k})$, with unitary $\Pi=-i C T=\tau_{y}$, which says that the filled and empty bands are symmetric with respect to the zero energy band.

To capture the topological structure of the Bloch bands, we define a projection operator onto the ground state of the system

$$
P(\mathbf{k})=\sum_{a \in \text { filled bands }}\left|u_{a}(\mathbf{k})\right\rangle\left\langle u_{a}(\mathbf{k})\right|
$$

and the ' $Q$ matrix'

$$
Q(\mathbf{k})=1-2 P(\mathbf{k})
$$


with the properties $Q^{\dagger}=Q$, and $Q^{2}=1$. The $Q$ matrix is a convenient simplification of the Hamiltonian as it has only two constant eigenvalues \pm 1 and can be obtained from the Hamiltonian by continuously deformation without closing the gap. Hence it's also called the 'flat band Hamiltonian'. As chiral operator sends a filled band to empty band, we have $\Pi P \Pi=1-P$, and $\Pi Q \Pi=-Q$. The presence of a chiral symmetry now allows us to bring the $Q$ matrix into block off-diagonal form in the chiral basis

$$
Q(\mathbf{k})=\left(\begin{array}{cc}
0 & q(\mathbf{k}) \\
q^{\dagger}(\mathbf{k}) & 0
\end{array}\right)
$$

Since $Q$ is unitary, its off-diagonal block $q(\mathbf{k})$ defines a map from the Brillouin zone to a space of unitary matrices with additional constraints imposed by PH and TR symmetries. In three dimension, the homotopy group of this map is isomorphic to the group of integers $\mathbb{Z}$. Topologically distinct phases in this symmetry class are classified by the winding number

$$
\nu=\frac{i}{48 \pi^{3}} \int_{B Z} \operatorname{Tr}\left[\left(q(\mathbf{k})^{-1} d q(\mathbf{k})\right)^{\wedge 3}\right] .
$$

In field theory, this winding number arises from the quantum anomaly of chiral symmetry, which originates from the Jacobian for the chiral transformation of the path integral measure for fermions[141]. There, an Atiyah-Singer index theorem can be proven[57]

$$
n_{R}-n_{L}=\nu
$$

where $n_{R, L}$ respectively denotes the number of eigenstate of the Dirac operator with vanishing eigenvalue with positive and negative chirality eigenvalues. Here, in the presence of a boundary, the translational invariance in its normal direction is broken, so that component of the momentum should be replaced by a derivative in the 
Hamiltonian. One can then solve differential equations to find gapless excitations localized at the boundary. These zero modes are required by Nambu doubling and PH symmetry to be their own Hermitian conjugates, i.e. Majorana fermions. What's nontrivial about these surface Majorana modes is that the correspondence between the net chirality of them and the bulk topological number protect them against symmetry respecting perturbations, including disorder. In other words, they are immune to Anderson localization, which says quantum states tend to be localized in the presence of strong impurities. This means that the system cannot be discretized and lattice version of the theory can only live on the boundary of a topological bulk. Historically, Anderson delocalization was hypothesized to be the defining property of TI/TSC, and one can identify topological phases in the ten-fold way by capturing the low-energy physic with effective field theory, non-linear sigma models (NL $\sigma \mathrm{Ms}$ ), and adding topological terms such as Wess-Zumino-Witten (WZW) terms[109].

\subsubsection{Collapse of $\mathbb{Z}$ classification under interaction}

SPT phases can exist in the presence of interactions, where one can have both fermionic and bosonic (spin) SPT phases. Interaction generally has two effects on topological phases. First, it allows for new topological phases that requires interactions, such as the Haldane phase of spin-1 Heisenberg antiferromagnetic chains. Second, it can reduce the non-interacting classification for certain symmetry classes, and change the boundary physics.

Recently it has been shown that under strong many-body interaction, as the surface state of 16 copies of a TSC can be gapped without breaking time reversal symmetry or introducing surface topological order[32, 74, 130, 111, 56, 95, 141]. This suggests the many-body extension allows a continuous path that connects 16 copies of a TSC to a trivial $s$-wave superconductor in three dimensions without breaking symmetry or closing the bulk gap. This reduces the $\mathbb{Z}$ classification of TSC in the 
single-body BCS description is reduced to $\mathbb{Z}_{16}$. In fact, the surface Majorana modes of any TSC can be gapped without breaking symmetries. However, there would generically be a residue topological order, unless $N$ is a multiple of 16 , that allows non-trivial anyonic excitations to live on the surface[32, 74]. As a result, these three dimensional (3D) bulk systems are still topologically distinct from a trivial state.

Similar phenomena were also seen in topological insulators[38, 100, 78, 94] in three dimensions and topological superconductors[61] in one dimension. Many-body interactions allow the surface Dirac mode of a topological insulator to acquire an energy gap without breaking time reversal or charge conservation symmetries. However a non-trivial surface topological order would be left behind.[128, 75, 20, 14] This indicates the bulk insulator still carries a non-trivial $\mathbb{Z}_{2}$ symmetry protected topology (SPT) even in the many-body framework. On the other hand, the $\mathbb{Z}$ classification of time reversal symmetric BDI superconductors in one dimension breaks down to $\mathbb{Z}_{8}$ in the presence of strong interaction[33, 34, 121, 21].

\subsubsection{Intrinsic topological order}

Symmetry protection is not an absolute requirement for distinct topological phases, systems with intrinsic topological order can be topologically distinct even in the absence of symmetry. Examples of systems with intrinsic topological order include fractional quantum Hall effect and Kitaev's toric code. Intrinsic topological orders are characterized by robust finite ground state degeneracy, non-local bulk excitations with fractional quantum numbers and fractional statistics and topologically protected gapless boundary excitations. These features have generated tremendous amount of interest in applications to topological quantum memory[25], fault-tolerant quantum computation[85], and perfect conduction devices.

The key difference between SPT phases and states with intrinsic topological order

lies in the entanglement. We define an equivalence relation between states that can be 
connected to each other with local unitary transformations. SPT phases are all equivalent to a direct product state. We call them short-range entangled (SRE) states, and all the other states long-range entangled (LRE) states. States with different intrinsic topological order are therefore characterized by different patterns of long-range entanglement, which cannot be transformed into each other with local unitary transformations. Long-range entanglement can be captured by topological entanglement entropy, defined as the constant part $\gamma$ of entanglement entropy $S=\alpha L+\gamma+O\left(L^{-1}\right)$.

The universal properties of the low-energy physics of LRE systems are described by an effective theory called topological quantum field theory (TQFT). A fundamental characteristic of TQFT is the quantum dimension $d_{a}$ of its quasiparticles excitations, anyons, which is 1 for Abelian anyons and $>1$ for non-Abelian anyons. It is related to the topological entanglement entropy by

$$
\gamma=\log \mathcal{D}
$$

where

$$
\mathcal{D}=\sqrt{\sum_{a} d_{a}^{2}}
$$

is called the total quantum dimension of the theory[30]. Quasiparticles in a TQFT are in one-to-one correspondence with the primary fields of a conformal field theory (CFT) defined on its edge. The quantum dimensions can be found from fusion rules among anyons, which correspond to operator product expansion (OPE) of primary fields in CFT,

$$
\phi_{a} \times \phi_{b}=\sum_{c} N_{a b}^{c} \phi_{c}
$$

The fusion coefficients $N_{a b}^{c}$ can be expressed in terms of the modular $S$ matrix trough Verlinde formula

$$
N_{a b}^{c}=\sum_{j} \frac{S_{a}^{j} S_{b}^{j} S_{j}^{c}}{S_{0}^{j}} .
$$


The modular $S$ matrix can be derived from the invariance of the partition function under modular transformation that exchanges space and time. The problem of finding anyon structure of a topological order is then reduced to computing the partition function, which is the sum of characters, in the representation theory of a centralextended symmetry algebra.

The topological order of a gapped symmetric surface of a topological insulator or superconductor was deduced mainly using vortex condensation or other topological field theory techniques. They do not specify the microscopic many-body surface gapping interactions that give rise to these exotic surface states. A pioneer work that addressed this issue was done by Fidkowski and Kitaev in Ref.[33] where they constructed explicit time reversal symmetric 4-fermion interactions that give an energy gap to eight boundary Majorana zero modes of a 1D TSC. Another insightful work was published by Mross, Essin and Alicea in Ref.[81] where they mimicked the surface Dirac mode of a topological insulator using a coupled wire model and wrote down explicit symmetric gapping interactions that lead to different gapped or gapless surface states.

Sliding Luttinger liquids[88, 28, 124, 114, 82] and coupled wire constructions[55] are immensely powerful in building two dimensional topological phases. They model 2D systems by arrays of coupled 1D chains, where interaction effects are more controlled and better understood. This theoretical technique has been frequently used in the study of fractional quantum Hall states[55, 117, 64, 73, 103], anyon models[89, 115], spin liquids[71, 41], (fractional) topological insulators[86, 65, 101, 102, 72, 70, 106] and superconductors[77, 112].

In chapter IV, we imitate the surface Majorana modes of a 3D topological superconductor using a coupled Majorana wire model, construct explicit 4-fermion interactions that lead to a finite excitation energy gap, and study the residue surface topological order. 


\section{Chapter II}

\section{Deformed Motzkin Spin Chain}

The deformation to increase/decrease entanglement entropy starts with the observation that Motzkin paths that reach a substantial height in the middle of the chain can contribute significant color correlations between the chain halves. The idea is illustrated in Fig. 2.1.

Remarkably, a suitable wave function, containing a superposition of colored Motzkin paths which prefers steep paths can be obtained as a frustration free and non-degenerate ground state of a Hamiltonian which is translational invariant in the bulk. Unfortunately, none of the ingredients in this statement are immediate. A generic change of the Hamiltonian presented in [80] may very easily either break frustration freeness or the non-degeneracy condition nor will it increase entanglement.
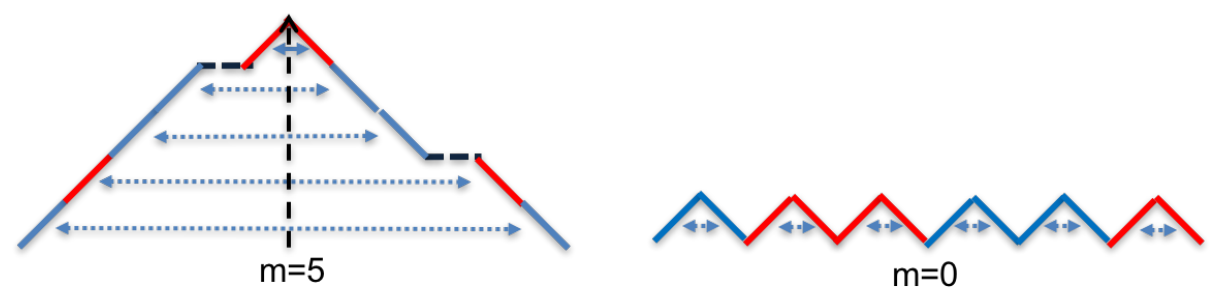

Figure 2.1: Motzkin paths require color correlations between the color of an up step and the color of the first step going down at the same height. The Motzkin path reaching height $m=5$ in the middle (left panel) contains more color correlations between the halves of the chain than a path of height $m=0$ (right panel). Favoring higher paths leads to more entanglement. 
Thus, we also need to show that we can do so in a way that the weight of these high paths is large enough as to overcome the contribution from more typical paths that reach height $\sqrt{n}$ at the middle of the chain

\subsection{A Frustration-Free Deformation}

\section{Theorem II.1. The Hamiltonian}

$$
H=|r\rangle\left\langle\left. r\right|_{1}+\mid l\right\rangle\left\langle\left. l\right|_{2 n}+\sum_{j=1}^{2 n-1} \Pi_{j, j+1},\right.
$$

defined on $\left(\mathbb{C}^{3}\right)^{\otimes 2 n}$ with

$$
\Pi_{j, j+1}=|\Phi\rangle\left\langle\left.\Phi\right|_{j, j+1}+\mid \Psi\right\rangle\left\langle\left.\Psi\right|_{j, j+1}+\mid \Theta\right\rangle\left\langle\left.\Theta\right|_{j, j+1},\right.
$$

where

$$
\begin{aligned}
|\Phi\rangle_{j, j+1} & =\cos \phi_{j+\frac{1}{2}}|0 l\rangle_{j, j+1}-\sin \phi_{j+\frac{1}{2}}|l 0\rangle_{j, j+1}, \\
|\Psi\rangle_{j, j+1} & =\cos \psi_{j+\frac{1}{2}}|0 r\rangle_{j, j+1}-\sin \psi_{j+\frac{1}{2}}|r 0\rangle_{j, j+1}, \\
|\Theta\rangle_{j, j+1} & =\cos \theta_{j+\frac{1}{2}}|00\rangle_{j, j+1}-\sin \theta_{j+\frac{1}{2}}|l r\rangle_{j, j+1}
\end{aligned}
$$

is frustration free and has a unique ground state with zero energy provided $\psi_{i}, \phi_{i}, \theta_{i} \in$ $(0, \pi / 2)$ satisfy relations

$$
\tan \theta_{i} \cot \phi_{i}=\tan \theta_{i+1} \tan \psi_{i+1}, \quad i=\frac{3}{2}, \frac{5}{2}, \frac{7}{2}, \ldots, 2 n-\frac{1}{2} .
$$

We first prove the uniqueness of the ground state (GS) first assuming the Hamiltonian is frustration free, and then show that the Hamiltonian is indeed frustration free under condition (2.2). 
Remark: When some of the angles equal an integer multiple of $\pi / 2$, the Hamiltonian is still frustration free, but may have a degenerate ground state.

Proof. (Uniqueness of GS) We look for a frustration-free ground state that will be annihilated by each of the terms in the Hamiltonian (3.1). We define the following $R, L, F$ moves and their inverses:

$$
|l 0\rangle \underset{R^{-1}}{\stackrel{R}{\rightleftharpoons}} \tan \phi|0 l\rangle, \quad|0 r\rangle \underset{L^{-1}}{\stackrel{L}{\rightleftharpoons}} \cot \psi|r 0\rangle, \quad|l r\rangle \underset{F^{-1}}{\stackrel{F}{\rightleftharpoons}} \tan \theta|00\rangle .
$$

We first note that if a ground state wave function contains a particular spin configuration (a "walk"), then the ground state wave function must contain as well a superposition of all states which can be obtained from it by the set of moves (2.3).

Indeed, at each neighboring two sites, the local spin state can be one of the nine possible configurations in $\{|l l\rangle,|r r\rangle,|r l\rangle,|0 l\rangle,|l 0\rangle,|0 r\rangle,|r 0\rangle,|00\rangle,|l r\rangle\}$, the first 3 of which are annihilated by the projectors $\Pi$ individually. The rest must form pairs $\sin \phi|0 l\rangle+\cos \phi|l 0\rangle, \sin \psi|0 r\rangle+\cos \psi|r 0\rangle$, and $\sin \theta|00\rangle+\cos \theta|l r\rangle$ to be annihilated by $|\Phi\rangle\langle\Phi|,| \Psi\rangle\langle\Psi|$, and $|\Theta\rangle\langle\Theta|$ respectively. Each of these superpositions corresponds to mixing between states related by the moves $R, L$, and $F$.

The process of generating additional walks starting from a given one is 'mixing' in that it can keep going on and on until all Motzkin walks are included in the superposition. To see this we construct the following procedures of relating Motzkin walks to the 'flat' mountain, i.e. the string of spins $000 \ldots 0$ : If the highest peak of the current mountain is of the type $l 0 \cdots r$ (i.e. a plateau), then keep applying $L$ and $R$ moves until it becomes $\operatorname{lr}$ (i.e. a hill), otherwise use the $F$ operation on the hill. Note that there are multiple choices of combinations of $L$ and $R$ moves that can be applied to make a mountain completely flat (See Fig. 2.2.) Given any Motzkin walk, we can represent it by a sequence of consecutive moves applied to it to get to the flat 


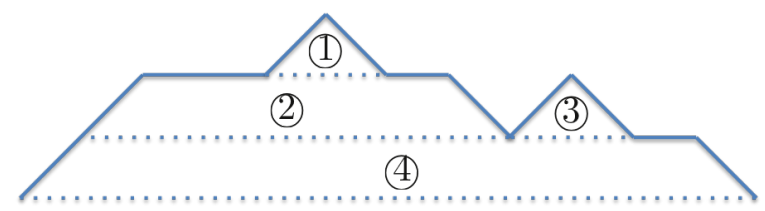

Figure 2.2: Iterative procedure to flatten a mountain to the ground, where steps 2 and 3 can be interchanged.

mountain, e.g. $\left|M_{1}\right\rangle=M_{1}|000 \ldots 0\rangle=\left(F_{i_{n}} \ldots R_{i_{3}} L_{i_{2}} L_{i_{1}}\right)^{-1}|000 \ldots 0\rangle$. So any two Motzkin walks are related by $\left|M_{2}\right\rangle=M_{2} M_{1}^{-1}\left|M_{1}\right\rangle$.

Similarly, it is easy to see that any walk which crosses below zero, or that does not return to zero at the end of the chain, can be transformed by the $R, L, F$ moves and their inverses into a walk that violates the boundary projectors. Therefore if a zero energy ground state does exist, then it will be the unique superposition of all Motzkin walks with weights determined by the tuned projectors.

It remains to be examined whether the aforementioned freedom in choosing the sequences of moves may result in ambiguities in the relative weights between Motzkin walks. It turns out that the tuning conditions (2.2) suffice to guarantee that a superposition of Motzkin walks can be written without ambiguities in the relative amplitudes. It can be seen from an observation of the local moves involving three adjacent sites illustrated in Fig. 2.3. The two ways to get $|000\rangle$ from $|l 0 r\rangle$ will give the same relative weight if and only if the mixing angles at two neighboring junctions satisfy the relation (2.2). The global version of this statement holds as well:

Proof. (Frustration Freeness) A plateau of width $d$, (that is, the number of 0 spins), is generated by one hill (or $F^{-1}$ move) and $d R^{-1}$ and $L^{-1}$ moves. (See Fig. 2.4.) Once the location of the hill is chosen, $R^{-1}\left(L^{-1}\right)$ only acts on its left (resp. right), and whether acting an $R^{-1}$ on the left first or an $L^{-1}$ on the right first doesn't affect the weight. So the weights are completely determined by the location of the hills that plateaus originate from at each level. The weights of the same plateau generated by 


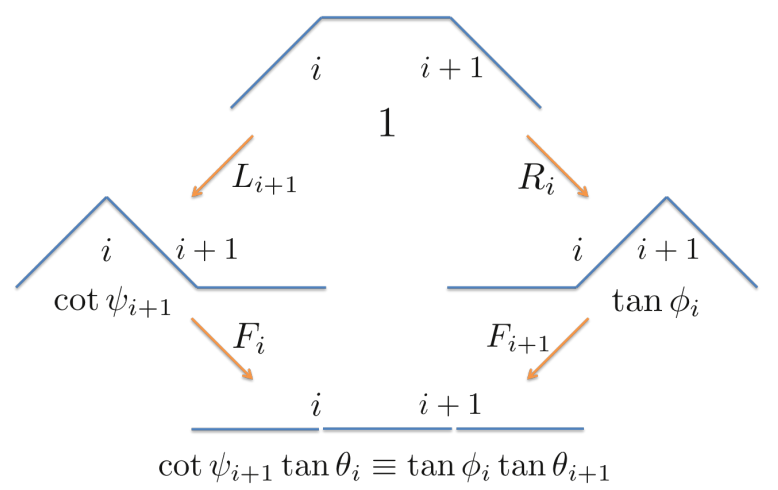

Figure 2.3: Two different sequences of moves to relate local state $|000\rangle$ to $|l 0 r\rangle$, and the relative weights of each state involved.

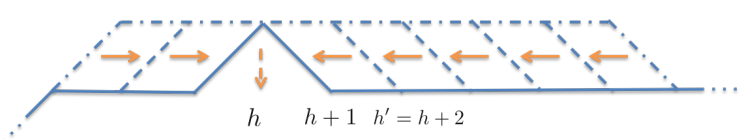

(a)

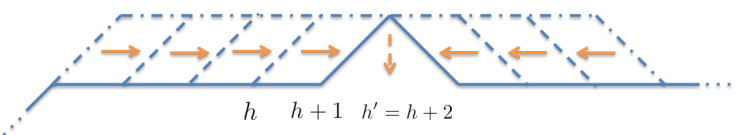

(b)

Figure 2.4: Different sequences of $L, R, F$ moves to get a hill (solid) from a starting plateau (dash dot) with intermediate plateaus after each move (dash). (a) Two different sequences of moves $R_{h-1} L_{h+1} L_{h+2} L_{h+3} R_{h-2} L_{h+4} L_{h+5}$ and $L_{h+1} L_{h+2} R_{h-1} L_{h+3} L_{h+4} L_{h+5} R_{h-2}$ with the same location of hill always give the same relative weight. (b) A sequence of moves with hill location different from those in (a) could generically give a different relative weight, except when relation (2.2) is satisfied. 
hills at location $h$ and $h^{\prime}$ are related by

$$
m(h)=\prod_{i=h}^{h^{\prime}-1} \frac{\tan \theta_{i} \cot \phi_{i}}{\tan \theta_{i+1} \tan \psi_{i+1}} m\left(h^{\prime}\right)=m\left(h^{\prime}\right) .
$$

Therefore the weight of each mountain is an invariant of the sequence of moves chosen to construct it from the flat mountain.

Furthermore, if two mountains are related directly to each other without passing through the flat mountain, by a sequence of $N$ moves, then each move in the sequence can be viewed as either a 'piling' move away from the flat mountain or a 'flattening' move towards it. So the intermediate mountains generated in this sequence each have definite weight $m_{1}, m_{2}, m_{3}, \ldots$, and the relative weight between these two mountains

$$
\frac{m_{A}}{m_{B}}=\frac{m_{A}}{m_{1}} \frac{m_{1}}{m_{2}} \frac{m_{2}}{m_{3}} \cdots \frac{m_{N}}{m_{B}}
$$

is an invariant. It follows that the relative weights between any two Motzkin walks are well-defined and conditions (2.2) is sufficient for Hamiltonian (3.1) to be frustration free.

\subsection{The Colorful Model}

Below we incorporate the colors in our model, and pick a particular, translationally invariant choice for the angles in Eq. (2.2), $\cot \phi_{i}=\tan \psi_{i}=t$. For simplicity, we further let $\cot \theta_{i}=t$. Now, all three moves $R^{-1}, L^{-1}$, and $F^{-1}$ in (2.3) change the weight of a Motzkin path by a factor of $t$ and increase the area below the mountain by exactly one unit. So the weight of each mountain is simply determined by the area below it. Thus, the weight of each mountain compared to the flat Motzkin path is given by $t^{\mathcal{A}(w)}$. The result is a ground state where the Motzkin paths are exponentially weighted according to the area under paths, rather than a uniform superposition. 
Theorem II.2. The following Hamiltonian, acting on a $2 n$ sites of a spin-s chain,

$$
H(s, t)=\Pi_{\text {boundary }}(s)+\sum_{j=1}^{2 n-1} \Pi_{j, j+1}(s, t)+\sum_{j=1}^{2 n-1} \Pi_{j, j+1}^{\text {cross }}(s)
$$

where

$$
\begin{aligned}
\Pi_{\text {boundary }}(s) & =\sum_{k=1}^{s}\left(\left|r^{k}\right\rangle\left\langle\left. r^{k}\right|_{1}+\mid l^{k}\right\rangle\left\langle\left. l^{k}\right|_{2 n}\right),\right. \\
\Pi_{j, j+1}(s, t) & =\sum_{k=1}^{s}\left(\left|\Phi(t)^{k}\right\rangle\left\langle\left.\Phi(t)^{k}\right|_{j, j+1}+\mid \Psi(t)^{k}\right\rangle\left\langle\left.\Psi(t)^{k}\right|_{j, j+1}+\mid \Theta(t)^{k}\right\rangle\left\langle\left.\Theta(t)^{k}\right|_{j, j+1}\right),\right. \\
\Pi_{j, j+1}^{\text {cross }}(s) & =\sum_{k \neq k^{\prime}}\left|l^{k} r^{k^{\prime}}\right\rangle\left\langle l^{k} r^{k^{\prime}}\right|,
\end{aligned}
$$

with

$$
\begin{aligned}
\left|\Phi^{k}(t)\right\rangle & =\frac{1}{\sqrt{1+t^{2}}}\left(\left|l^{k} 0\right\rangle-t\left|0 l^{k}\right\rangle\right), \\
\left|\Psi^{k}(t)\right\rangle & =\frac{1}{\sqrt{1+t^{2}}}\left(\left|0 r^{k}\right\rangle-t\left|r^{k} 0\right\rangle\right), \\
\left|\Theta^{k}(t)\right\rangle & =\frac{1}{\sqrt{1+t^{2}}}\left(\left|l^{k} r^{k}\right\rangle-t|00\rangle\right),
\end{aligned}
$$

has a unique zero energy ground state

$$
|G S\rangle=\frac{1}{\mathcal{N}} \sum_{\substack{w \in\{\text { s-colored } \\ \text { Motzkin walks }\}}} t^{\mathcal{A}(w)}|w\rangle
$$

where $\mathcal{A}(w)$ denotes the area below the Motzkin walk $w$, and $\mathcal{N}$ is a normalization factor.

A caricature of the resulting ground state is shown in Fig. 2.5. 


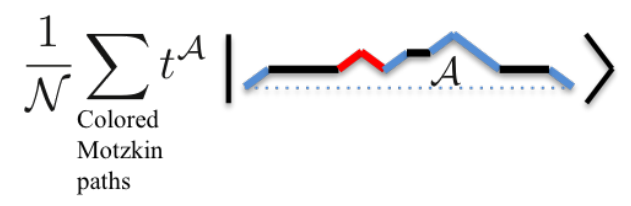

Figure 2.5: A caricature of the ground state of our model.

\subsection{Entanglement Entropy}

In the Schmidt decomposition of the ground state (2.7), the coloring of the unpaired spins in the second half of the system is completely determined by those the first half (see Fig. 2.1). The Schmidt decomposition is written as:

$$
|G S\rangle=\sum_{m=0}^{n} \sqrt{p_{n, m}} \sum_{x \in\left\{l^{1}, l^{2} . l^{s}\right\}^{m}}\left|\hat{C}_{0, m, x}\right\rangle_{1 . . n} \otimes\left|\hat{C}_{m, 0, \bar{x}}\right\rangle_{n+1 . .2 n}
$$

where $\left|\hat{C}_{p, q, x}\right\rangle$ is a weighted superposition of states in $\left\{0, u^{1}, \ldots, u^{s}, d^{1}, \ldots, d^{s}\right\}^{n}$ with $p$ excess down moves, $q$ excess up moves and a particular coloring $x$ of the unmatched up moves, such that $\left\langle G S\left|\left(\left|\hat{C}_{0, m, x}\right\rangle_{1 . . n} \otimes\left|\hat{C}_{m, 0, \bar{x}}\right\rangle_{n+1 . .2 n}\right) \neq 0\right.\right.$, and $\bar{x}$ is the coloring in the second half of the chain that matches $x$. The decomposition gives the Schmidt number $p_{n, m}(s, t)=\frac{M_{n, m}^{2}(s, t)}{N_{n}(s, t)}$, with

$$
M_{n, m}(s, t) \equiv \sum_{i=0}^{\frac{n-m}{2}} s^{i} \sum_{w \in D_{n, m, i}} t^{2 \mathcal{A}(w)} ; N_{n} \equiv \sum_{m=0}^{n} s^{m} M_{n, m}^{2}
$$

Here $D_{n, m, i}=$ " 1 st half of Motzkin walks with $i$ paired spins stopping at $(n, m)$ ". Notice that the extra factor of two in the exponent of $t$ is there so that the $\left|\hat{C}_{p, q, x}\right\rangle$ and $\left|\hat{C}_{p, q, \bar{x}}\right\rangle$ form an orthonormal basis. The entanglement entropy of the half chain in the ground state is given by

$$
S_{n}(s, t)=-\sum_{m=0}^{n} s^{m} p_{n, m}(s, t) \log p_{n, m}(s, t)
$$




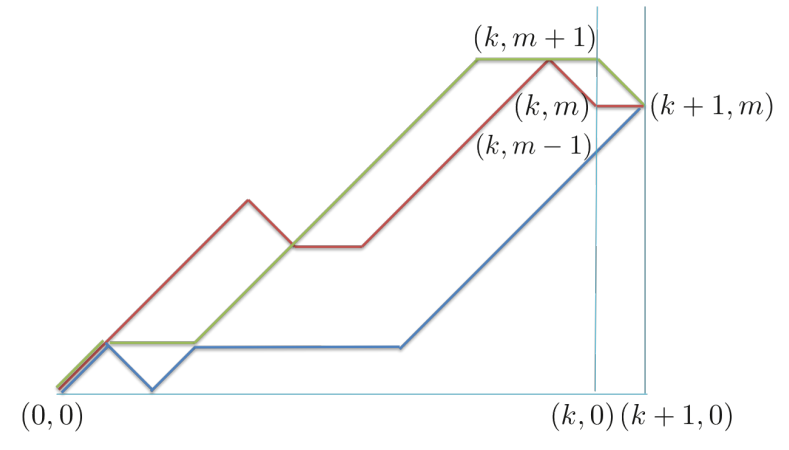

Figure 2.6: Three representative paths of different heights at position $k$ to generate a path that ends with height $m$ at position $k+1$. The increments in the area below the paths are the areas sandwiched between two vertical lines, which are $m+\frac{1}{2}, m, m-\frac{1}{2}$ respectively for red, green and blue paths.

To study the asymptotic scaling of $S_{n}(s, t)$ with the system size when $t \neq 1$, we note the following recursion relations of $M_{n, m}$ as a function of $m$ :

$$
\begin{aligned}
M_{k+1, k+1} & =t^{2 k+1} M_{k, k}, \\
M_{k+1, k} & =t^{2 k} M_{k, k}+t^{2 k-1} M_{k, k-1}, \\
M_{k+1, m} & =s t^{2 m+1} M_{k, m+1}+t^{2 m} M_{k, m}+t^{2 m-1} M_{k, m-1} \\
M_{k+1,0} & =s t M_{k, 1}+M_{k, 0} .
\end{aligned}
$$

These can be easily seen from the possible ways to arrive at a particular destination and the increment of the area below each path as illustrated in Fig. 2.6. The third equation above is valid for $0<m<k$.

Starting from the seed value $M_{0,0}=1$, by using the recurrence relations repetitively, one can calculate the values of $M_{n, m}$ and Schmidt numbers for any $m$ and calculate the entanglement entropy $S_{n}$. To get a better handle on the type of distribution the relations lead to, we find it convenient to view the recursion evolution as a process of increasing/decreasing $m$ while $n$ is viewed as discrete 'time'. We will show below that for large enough $n$, the distribution associated with the $M_{n, m}$ essentially propagates ballistically (as a function of $n$ ), with very little spread. This property 
will establish that the typical height at the middle of a $2 n$ chain scales linearly with $n$.

Before exhibiting the proof, we need to develop a few preliminary steps. We encode the distributions $M_{n, m}$ as coefficients of wavefunctions defined on the set $|m\rangle, m=0,1,2, \ldots$ as

$$
\left|\mathcal{M}_{n}\right\rangle=\sum_{m=0}^{\infty} M_{n, m}|m\rangle, \quad M_{n, m}=0 \text { if } m>n
$$

We define the following 'shift' and 'height' operators, which we will use in describing the 'evolution' of the distribution $M_{n, m}$ as function of 'time' $n$.

$$
\begin{aligned}
& \mathcal{S}|m\rangle=|m-1\rangle, \quad|-1\rangle=0 ; \\
& \mathcal{H}|m\rangle=m|m\rangle .
\end{aligned}
$$

Explicitly, $t^{2 \mathcal{H}}, \mathcal{S}, \mathcal{S}^{\dagger}$ act on $\left|\mathcal{M}_{n}\right\rangle$ as follows.

$$
\begin{aligned}
t^{2 \mathcal{H}}\left|\mathcal{M}_{n}\right\rangle & =\sum_{m=0}^{\infty} M_{n, m} t^{2 m}|m\rangle, \\
\mathcal{S}\left|\mathcal{M}_{n}\right\rangle & =\sum_{m=1}^{\infty} M_{n, m}|m-1\rangle=\sum_{m=0}^{\infty} M_{n, m+1}|m\rangle, \\
\mathcal{S}^{\dagger}\left|\mathcal{M}_{n}\right\rangle & =\sum_{m=0}^{\infty} M_{n, m-1}|m\rangle .
\end{aligned}
$$

As remarked above, we aim to show that for large enough $n,\left|\mathcal{M}_{n+1}\right\rangle \propto \mathcal{S}^{\dagger}\left|\mathcal{M}_{n}\right\rangle$, describing essentially ballistic propagation with no spread. For the recurrence relation (2.14) to be satisfied, we require

$$
\begin{aligned}
& M_{n+1, m}=\left\langle m \mid \mathcal{M}_{n+1}\right\rangle=s t^{2 m+1}\left\langle m+1 \mid \mathcal{M}_{n}\right\rangle+t^{2 m}\left\langle m \mid \mathcal{M}_{n}\right\rangle+t^{2 m-1}\left\langle m-1 \mid \mathcal{M}_{n}\right\rangle \\
& =\left\langle m\left|s t^{2 \mathcal{H}+1} \mathcal{S}+t^{2 \mathcal{H}}+t^{2 \mathcal{H}-1} \mathcal{S}^{\dagger}\right| \mathcal{M}_{n}\right\rangle .
\end{aligned}
$$


Therefore,

$$
\left|\mathcal{M}_{n+1}\right\rangle=t^{2 \mathcal{H}}\left(s t \mathcal{S}+1+\frac{1}{t} \mathcal{S}^{\dagger}\right)\left|\mathcal{M}_{n}\right\rangle
$$

Using the relations,

$$
\begin{aligned}
t^{2 \mathcal{H}} \mathcal{S} & =t^{-2} \mathcal{S} t^{2 \mathcal{H}} \\
t^{2 \mathcal{H}} \mathcal{S}^{\dagger} & =t^{2} \mathcal{S}^{\dagger} t^{2 \mathcal{H}}
\end{aligned}
$$

we have

$$
t^{2 k \mathcal{H}}\left(s t \mathcal{S}+1+\frac{1}{t} \mathcal{S}^{\dagger}\right)=\left(s t^{-(2 k-1)} \mathcal{S}+1+t^{2 k-1} \mathcal{S}^{\dagger}\right) t^{2 k \mathcal{H}},
$$

and

$$
\begin{aligned}
\left|\mathcal{M}_{n}\right\rangle & =\left[t^{2 \mathcal{H}}\left(s t \mathcal{S}+1+\frac{1}{t} \mathcal{S}^{\dagger}\right)\right]^{n}\left|\mathcal{M}_{0}\right\rangle \\
& =\overrightarrow{\mathcal{K}} \prod_{k=1}^{n}\left(s t^{-(2 k-1)} \mathcal{S}+1+t^{2 k-1} \mathcal{S}^{\dagger}\right)|0\rangle
\end{aligned}
$$

where $\overrightarrow{\mathcal{K}}$ denotes ordering the multiplications in the product such that factors with greater $k$ value is on the right. It is now evident that the factors in the product above are dominated by the $\mathcal{S}^{\dagger}$ term for large $k$, giving us "ballistic" evolution with $n$.

\subsubsection{Extensive entropy for $t>1, s>1$}

Lemma II.1. Let $m^{*}$ be such that $\sup _{m} M_{n, m}=M_{n, m^{*}}$, then $\exists N_{0}<n$, such that when $t>1, m^{*} \in\left[n-2 N_{0}, n\right]$.

Proof. Let

$$
\left|\mathcal{M}_{n}^{\prime}\right\rangle=\overrightarrow{\mathcal{K}} \prod_{k=N_{0}+1}^{n}\left(s t^{-(2 k-1)} \mathcal{S}+1+t^{2 k-1} \mathcal{S}^{\dagger}\right)|0\rangle .
$$

Note that

$$
t^{-(2 k-1)}\left\|1+s t^{-(2 k-1)} \mathcal{S}\right\|_{1} \leq t^{-(2 k-1)}+s t^{-2(2 k-1)} \equiv c_{k},
$$


so that:

$$
\left\|s t^{-(2 k-1)} \mathcal{S}+1+t^{2 k-1} \mathcal{S}^{\dagger}\right\|_{1}<t^{2 k-1}\left(1+c_{k}\right)
$$

we thus have

$$
\begin{gathered}
\| t^{-\sum_{k=N_{0}+1}^{n}(2 k-1)}\left|\mathcal{M}_{n}^{\prime}\right\rangle-\left|n-N_{0}\right\rangle \|_{1} \leq \prod_{k=N_{0}+1}^{n}\left(c_{k}+1\right)-1<e^{\sum_{k=N_{0}+1}^{\infty} c_{k}}-1 \\
=e^{t^{-4 N_{0}} \frac{s t^{2}+t^{2 N_{0}+1}+t^{2 N_{0}+3}}{t^{4}-1}}-1<e^{t^{-2 N_{0}} \frac{3 s t^{3}}{t^{4}-1}}-1 \equiv f(s, t)^{t^{-2 N_{0}}}-1 .
\end{gathered}
$$

The first inequality on the left follows from noting that $\left|n-N_{0}\right\rangle$ appears in $t^{-\sum_{k=N_{0}+1}^{n}(2 k-1)}\left|\mathcal{M}_{n}^{\prime}\right\rangle$ with coefficient 1 , and is exactly canceled. We have also used that $x+1 \leq e^{x}$. Next,

$$
\begin{aligned}
& \| t^{-\sum_{k=1}^{n}(2 k-1)}\left|\mathcal{M}_{n}\right\rangle-\overrightarrow{\mathcal{K}} \prod_{k=1}^{N_{0}}\left(s t^{-2(2 k-1)} \mathcal{S}+t^{-(2 k-1)}+\mathcal{S}^{\dagger}\right)\left|n-N_{0}\right\rangle \|_{1} \\
\leq & \left\|\overrightarrow{\mathcal{K}} \prod_{k=1}^{N_{0}}\left(s t^{-2(2 k-1)} \mathcal{S}+t^{-(2 k-1)}+\mathcal{S}^{\dagger}\right)\right\|_{1} \| t^{-\sum_{k=N_{0}+1}^{n}(2 k-1)}\left|\mathcal{M}_{n}^{\prime}\right\rangle-\left|n-N_{0}\right\rangle \|_{1} \\
<\left(f(s, t)^{t^{-2 N_{0}}}-1\right) & \prod_{k=1}^{N_{0}}\left(1+c_{k}\right)<\left(f(s, t)^{t^{-2 N_{0}}}-1\right) e^{\sum_{k=1}^{N_{0}}} c_{k}<\left(f(s, t)^{t^{-2 N_{0}}}-1\right) f(s, t) .
\end{aligned}
$$

Let

$$
M_{n, m}^{\prime}=\left\langle m\left|\overrightarrow{\mathcal{K}} \prod_{k=1}^{N_{0}}\left(s t^{-2(2 k-1)} \mathcal{S}+t^{-(2 k-1)}+\mathcal{S}^{\dagger}\right)\right| n-N_{0}\right\rangle,
$$

then clearly $M_{n, m}^{\prime}=0$ for $m<n-2 N_{0}$. If we choose

$$
N_{0}= \begin{cases}0 & f(s, t)<\frac{1+\sqrt{5}}{2}, \\ -\frac{\log \frac{\log \left(f^{-1}(s, t)+1\right)}{\log f(s, t)}}{2 \log t}, & \text { otherwise }\end{cases}
$$

then

$$
\| t^{-n^{2}}\left|\mathcal{M}_{n}\right\rangle-\sum_{m=n-2 N_{0}}^{n} M_{n, m}^{\prime}|m\rangle \|_{1}<1=M_{n, n}^{\prime} \leq \sup _{m} M_{n, m}^{\prime}
$$


Therefore $\exists m^{*} \in\left[n-2 N_{0}, n\right]$, such that $M_{n, m^{*}} \geq M_{n, m}$ for all $m$.

Lemma II.1 shows that the peak of the $M_{n, m}$ distribution is always within a finite distance from $n$. Essentially, the bulk of the distribution travels with velocity 1 .

Theorem II.3. In the state (2.7), when $t>1$, the entanglement entropy of sites $1 \ldots n$, is bounded below as $S_{n}>n \log s+$ const. for all $n$, where const. is an $n$ independent constant.

Proof. We separate a linear term from $S_{n}$ as follows (below we supress the $n$ index in $\left.M_{n, m}\right)$ :

$$
\begin{gathered}
S_{n}=-\sum_{m=0}^{n} s^{m} p_{m} \log \frac{M_{m}^{2}}{\sum_{m^{\prime}=0}^{n} s^{m^{\prime}} M_{m^{\prime}}^{2}}> \\
=-\sum_{m=0}^{n} s^{m} p_{m} \log \frac{M_{m}^{2}}{s^{m} M_{m}^{2}} \\
=\sum_{m=0}^{n} s^{m} p_{m} m \log s=\sum_{l=0}^{n} s^{n-l} p_{n-l}(n-l) \log s \\
=n \log s-\log s \sum_{l=0}^{n} \frac{s^{n-l} M_{n-l}^{2}}{\sum_{m^{\prime}=0}^{n} s^{m^{\prime}} M_{m^{\prime}}^{2}} l
\end{gathered}
$$

Taking $m^{*}$ such that $\sup _{m} M_{n, m}=M_{n, m^{*}}$ and using lemma III.1, we see that

$$
\begin{aligned}
& \sum_{l=0}^{n} \frac{s^{n-l} M_{m^{*}}^{2}}{\sum_{m^{\prime}=0}^{n} s^{m^{\prime}} M_{m^{\prime}}^{2}} l<\sum_{l=0}^{n} \frac{s^{n-l} M_{m^{*}}^{2}}{s^{m^{*}} M_{m^{*}}^{2}} l=s^{n-m^{*}} \sum_{l=0}^{n} s^{-l} l \\
& <s^{2 N_{0}} \sum_{l=0}^{n} s^{-l} l<s^{2 N_{0}} \sum_{l=0}^{\infty} s^{-l} l=\frac{s^{2 N_{0}+1}}{(s-1)^{2}} .
\end{aligned}
$$

Therefore, the remainder term on the right hand side of (3.28) is bounded.

\subsubsection{Bounded entropy for $t<1$}

When $t<1$ we expect the Motzkin paths with the lowest area to be exponentially preferred. In particular, the flat Motzkin path that has zero area has a vanishing contribution to entropy, and thus we expect the entanglement entropy to be substantially 
reduced. In fact, it turns out that the for any value $t<1$ the entropy is bounded, independently of the size of the system $2 n$ :

Theorem II.4. When $0<t<1, s \geq 1$, there exists a constant $C(s, t)$ independent of the system size $n$, such that for any $n, S_{n}<C(s, t)$.

Remark: Note that theorem holds both for the colored and uncolored case $s=1$.

For the theorem to hold, the exponential growth in contribution to entropy from the possible colorings of higher paths should be overwhelmed by the exponential price in area. Technically, we need the quantities $M_{m}^{2}$ to decrease faster than the rate $s^{m}$ grows in order to make $p_{m}$ decrease exponentially.

To highlight this feature we first define

$$
\tilde{M}_{n, m}=s^{\frac{m}{2}} M_{n, m}, \tilde{p}_{n, m}=\frac{\tilde{M}_{n, m}^{2}}{\sum_{m=0}^{n} \tilde{M}_{n, m}^{2}} .
$$

Substitution into (??) gives the relation

$$
\tilde{M}_{n+1, m}=\sqrt{s} t^{2 m+1} \tilde{M}_{n, m+1}+t^{2 m} \tilde{M}_{n, m}+\sqrt{s} t^{2 m-1} \tilde{M}_{n, m-1},
$$

for $m \in[1, n-1]$.

To prove the entropy is bounded, we need the following lemmas.

\section{Lemma II.2.}

$$
\tilde{M}_{n+1, m}^{2}>\tilde{M}_{n, m}^{2}
$$


Proof. From (3.22), we have

$$
\begin{gathered}
\left|\mathcal{M}_{n+1}\right\rangle=\overrightarrow{\mathcal{K}} \prod_{k=1}^{n+1}\left(s t^{-(2 k-1)} \mathcal{S}+1+t^{2 k-1} \mathcal{S}^{\dagger}\right)|0\rangle \\
=\overrightarrow{\mathcal{K}} \prod_{k=1}^{n}\left(s t^{-(2 k-1)} \mathcal{S}+1+t^{2 k-1} \mathcal{S}^{\dagger}\right)\left(s t^{-(2 n+1)} \mathcal{S}+1+t^{2 n+1} \mathcal{S}^{\dagger}\right)|0\rangle \\
=\left|\mathcal{M}_{n}\right\rangle+\overrightarrow{\mathcal{K}} \prod_{k=1}^{n}\left(s t^{-(2 k-1)} \mathcal{S}+1+t^{2 k-1} \mathcal{S}^{\dagger}\right)\left(s t^{-(2 n+1)} \mathcal{S}+t^{2 n+1} \mathcal{S}^{\dagger}\right)|0\rangle
\end{gathered}
$$

The last term on the RHS of the equation contains non-zero contributions for all states $|m\rangle$, with $m=0, . . n+1$, and we have:

$$
\begin{aligned}
& M_{n+1, m}>M_{n, m}, \\
& \tilde{M}_{n+1, m}>\tilde{M}_{n, m} \quad \forall m \geq 0, n \geq 1 .
\end{aligned}
$$

And the Lemma follows.

Next we establish the following bound on $\tilde{p}_{n, m}$ :

\section{Lemma II.3.}

$$
\tilde{p}_{n, m}<9 s t^{4 m-2} \text {. }
$$

Proof. By definition of $\tilde{p}_{n, m}$, and the recursion relation (3.30),

$$
\begin{aligned}
\tilde{p}_{n, m} & =\frac{\tilde{M}_{n, m}^{2}}{\sum_{m=0}^{n} \tilde{M}_{n, m}^{2}}= \\
& =\frac{\left(\sqrt{s} t^{2 m+1} \tilde{M}_{n-1, m+1}+t^{2 m} \tilde{M}_{n-1, m}+\sqrt{s} t^{2 m-1} \tilde{M}_{n-1, m-1}\right)^{2}}{\sum_{m=0}^{n} \tilde{M}_{n, m}^{2}} \\
& =\frac{t^{4 m}\left(\sqrt{s} t \tilde{M}_{n-1, m+1}+\tilde{M}_{n-1, m}+\sqrt{s} t^{-1} \tilde{M}_{n-1, m-1}\right)^{2}}{\sum_{m=0}^{n} \tilde{M}_{n, m}^{2}} \\
& \leq t^{4 m} \frac{\left(3 \sqrt{s} t^{-1} \max \left\{t^{2} \tilde{M}_{n-1, m+1}, \frac{t}{\sqrt{s}} \tilde{M}_{n-1, m}, \tilde{M}_{n-1, m-1}\right\}\right)^{2}}{\sum_{m=0}^{n} \tilde{M}_{n, m}^{2}} \\
& \leq 9 t^{4 m} \frac{s}{t^{2}} \frac{\max \left\{\tilde{M}_{n, m+1}^{2}, \tilde{M}_{n, m}^{2}, \tilde{M}_{n, m-1}^{2}\right\}}{\sum_{m=0}^{n} \tilde{M}_{n, m}^{2}}<9 t^{4 m} \frac{s}{t^{2}} .
\end{aligned}
$$


Lemma III.2 was used in the last line.

We now have the ingredients to prove theorem II.4:

Proof. (Theorem II.4) Using Lemma III.3 we see that when

$$
m>m_{0} \equiv\left[\frac{\log \left(\frac{1}{9 e} \frac{t^{2}}{s}\right)}{4 \log t}\right]+1
$$

we have

$$
\tilde{p}_{n, m}<9 s t^{4 m-2}<\frac{1}{e}
$$

It is easy to check that the function $-x \log (x)$ is monotonically increasing when $x \in\left(0, \frac{1}{e}\right)$, in other words, for $m>m_{0}$,

$$
\tilde{p}_{n, m}<9 s t^{4 m-2}<\frac{1}{e} \Longrightarrow-\tilde{p}_{n, m} \log \tilde{p}_{n, m}<-9 s t^{4 m-2}\left(\log \left(\frac{9 s}{t^{2}}\right)+4 m \log t\right)
$$

Therefore

$$
\begin{aligned}
S_{n}= & -\sum_{m=0}^{n} \tilde{p}_{n, m} \log \tilde{p}_{n, m}+\log s \sum_{m=0}^{n} \tilde{p}_{n, m} m \\
< & -\sum_{m=0}^{m_{0}} \tilde{p}_{n, m} \log \tilde{p}_{n, m}-\sum_{m=m_{0}+1}^{\infty} 9 s t^{4 m-2}\left(\log \left(\frac{9 s}{t^{2}}\right)+4 m \log t\right)+\log s \sum_{m=0}^{\infty} 9 s t^{4 m-2} m \\
< & \frac{m_{0}+1}{e}-\frac{9 s t^{4 m_{0}+2}}{1-t^{4}} \log \left(\frac{9 s}{t^{2}}\right)-\frac{18 s t^{4 m_{0}+2}\left(m_{0}\left(1-t^{4}\right)+1\right)}{\left(t^{4}-1\right)^{2}} 2 \log t \\
& +\frac{9 s t^{2}}{\left(t^{4}-1\right)^{2}} \log s \equiv C(s, t),
\end{aligned}
$$

where we used $\sup _{x \in(0,1)}-x \log (x)=e^{-1}$ for entropy terms with $m \leq m_{0}$ in the last inequality. 


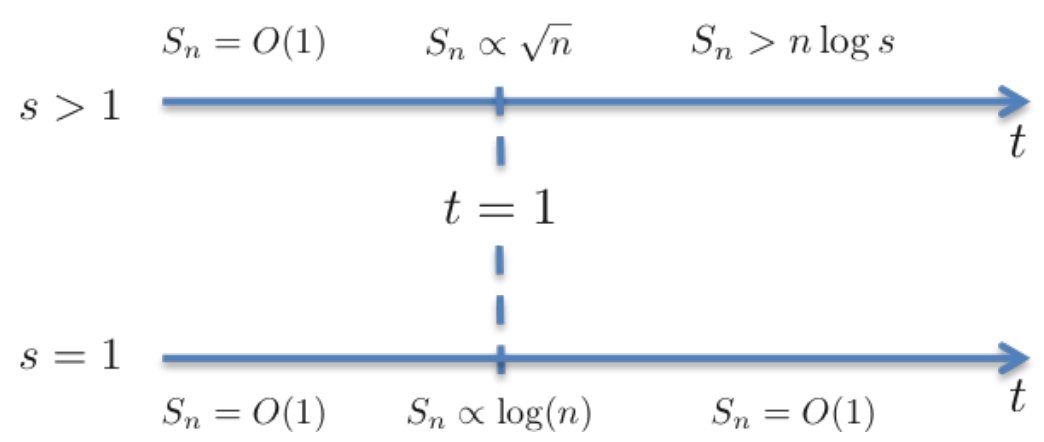

Figure 2.7: Entanglement entropy of the first $n$ sites in a chain of $2 n$ sites for various phases of the colored and uncolored area-weighted Motzkin state.

\subsection{A novel quantum phase transition}

The abrupt change in scaling of entanglement entropy proven above, combined with previous results shown in ref. $[15,80]$ can be summarized in the phase diagram in Fig. 2.7. Additional evidence can be drawn from sudden change in scalings of spectral gap. In the next chapter, we will rigorously prove for the deformed Fredkin chain upper bounds on the scaling of gap for $t>1, s>1$ phase and $t>1, s=1$ phase, showing both of them vanishes in the thermodynamic limit. The same argument goes for the deformed Motzkin chain. Besides, numerical studies in ref. [12] shows that the $t<1$ phase appears to be gapped, in agreement with our conjecture based on the $t \rightarrow 0$ limit.

We call this phase transition a novel quantum phase transition out of the following considerations. On one hand, it happens at zero temperature and is a result of coefficient in the Hamiltonian changing near a critical value. On the other hand, the ground state itself does not go through and abrupt change, and no level crossing is involved, falling out of the definition of quantum phase transition given in chapter I. In addition, while bounded entanglement entropy usually indicate a non-vanishing spectral gap, our $t>1, s=1$ with bounded entanglement entropy will be shown to be gapless in the next chapter. 


\section{Chapter III}

\section{Deformed Fredkin Spin Chain}

In this chapter, we apply the idea from the previous chapter to a half-integer spin version of the Motzkin model, called Fredkin spin chain. In addition, we further explore the degrees of freedom in frustration free deformation of the Hamiltonian. Inspired by the more mathematically inclined proof given in ref. [68], and numerical results in ref. [12], we give a rigorous and intuitive prove on scalings of the spectral gap omitted in the previous chapter.

\subsection{Multi-parameter Hamiltonian and ground state}

Following the method in the previous chapter, we introduce a parameter $t$ that

deforms the Fredkin Hamiltonian of [24, 104] while remaining frustration free. The Hamiltonian is given by:

$$
H(s, t)=H_{F}(s, t)+H_{X}(s)+H_{\partial}(s)
$$

where

$$
H_{F}(s, t)=\sum_{j=2}^{2 n-1} \sum_{c_{1}, c_{2}, c_{3}=1}^{s}\left(\left|\phi_{j, A}^{c_{1}, c_{2}, c_{3}}\right\rangle\left\langle\phi_{j, A}^{c_{1}, c_{2}, c_{3}}|+| \phi_{j, B}^{c_{1}, c_{2}, c_{3}}\right\rangle\left\langle\phi_{j, B}^{c_{1}, c_{2}, c_{3}}\right|\right)
$$




$$
\begin{aligned}
& H_{X}(s)= \sum_{j=1}^{2 n-1}\left[\sum_{c_{1} \neq c_{1}}\left|\uparrow_{j}^{c_{1}} \downarrow_{j+1}^{c_{2}}\right\rangle\left\langle\uparrow_{j}^{c_{1}} \downarrow_{j+1}^{c_{2}}\right|\right. \\
&\left.+\frac{1}{2} \sum_{c_{1}, c 2=1}^{s}\left(\left|\uparrow_{j}^{c_{1}} \downarrow_{j+1}^{c_{1}}\right\rangle-\left|\uparrow_{j}^{c_{2}} \downarrow_{j+1}^{c_{2}}\right\rangle\right)\left(\left\langle\uparrow_{j}^{c_{1}} \downarrow_{j+1}^{c_{1}}\right|-\left\langle\uparrow_{j}^{c_{2}} \downarrow_{j+1}^{c_{2}}\right|\right)\right],
\end{aligned}
$$

and

$$
H_{\partial}(s)=\sum_{c=1}^{s}\left(\left|\downarrow_{1}^{c}\right\rangle\left\langle\downarrow_{1}^{c}|+| \uparrow_{2 n}^{c}\right\rangle\left\langle\uparrow_{2 n}^{c}\right|\right)
$$

The projectors in $H_{F}$ are defined using:

$$
\begin{aligned}
& \left|\phi_{j, A}^{c_{1}, c_{2}, c_{3}}\right\rangle=\frac{1}{\sqrt{1+\left|t_{A, j}\right|^{2}}}\left(\left|\uparrow_{j}^{c_{1}} \uparrow_{j+1}^{c_{2}} \downarrow_{j+2}^{c_{3}}\right\rangle-t_{A, j}\left|\uparrow_{j}^{c_{2}} \downarrow_{j+1}^{c_{3}} \uparrow_{j+2}^{c_{1}}\right\rangle\right)
\end{aligned}
$$

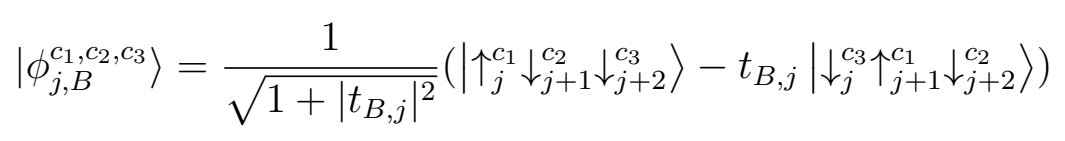

with the condition that $t_{j}^{B}=t_{j-1}^{A}$.

The Fredkin gate projectors in $H_{F}$ allows a pair of $\uparrow \downarrow$ neighboring spins (with the same color enforced by the first term in $H_{X}$ ) to move freely around its left or right third neighbor and still appear in the ground state superposition, but now with a different probability amplitude. The second term in $H_{X}$ ensures that otherwise identical Dyck paths with different coloring have the same weight. And the boundary term $H_{\partial}$ (together with the Fredkin projectors) penalizes paths that go below 0 at any point along the chain. Notice that analogous to [146], the simplest choice is a parameter $t=t_{A}=t_{B}$ being the same in the two projectors of $H_{F}$ is the one employed in $[105,122]$, but is only a subset of the parameter space that leaves the Hamiltonian frustration free. More generally, we introduce parameters $t^{A}$ and $t^{B}$, for the two projectors in $H_{F}$. Then any set of $\left\{t_{j}^{A}, t_{j}^{B}\right\}$ that satisfies the condition $t_{j}^{A}=t_{j+1}^{B}$ for all $j$ 's would guarantee the Hamiltonian to be frustration free ${ }^{1}$. The

\footnotetext{
${ }^{1}$ Note that there is no parallel condition $t_{j}^{B}=t_{j+1}^{A}$.
} 


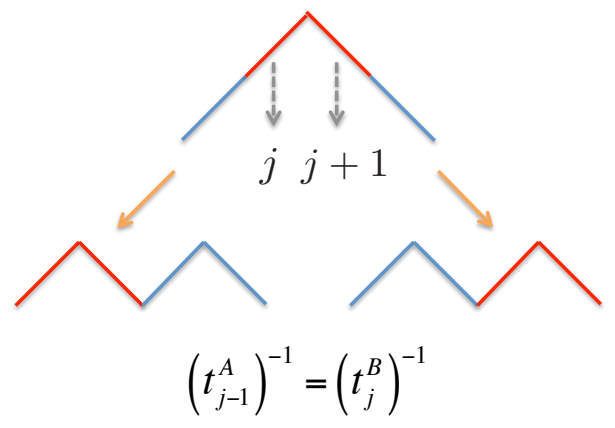

Figure 3.1: Different ways of "flattening" a hill must have the same amplitude. Note that the colors have been interchanged during the procedure, this, however, is not an obstruction as all colorings appear with the same amplitude.

point is illustrated in Fig. 3.1. In particular, we may specify a Hamiltonian with a frustration-free ground state by picking any set of the $t_{j}^{A}$ parameters.

We now want to characterize the ground state of the system. First, let us denote $h(l)$ to be the height of the Dyck path after step $l$, that is, for a spin configuration $|w\rangle$ describing a Dyck path,

$$
\sum_{c=1}^{s} \sum_{j=1}^{l} \sigma_{j, c}^{z}|w\rangle=h(l)|w\rangle
$$

where $\sigma_{j, c}^{z}$ is the Pauli matrix giving \pm 1 if spin $j$ is in state $\uparrow_{j}^{c}$ or $\downarrow_{j}^{c}$, respectively. The height function is illustrated for a generic Dyck path in Fig. 3.2. To find the relative amplitude of this spin configuration as compared with the lowest possible spin configuration, we use successively the Fredkin moves to $|\uparrow \uparrow \downarrow\rangle \longrightarrow t_{A}|\uparrow \downarrow \uparrow\rangle$ to "flatten" the hill. The process is described in Fig. 3.3. In this way, the weight of each Dyck paths is related to the weight of the lowest height, $|\uparrow \downarrow \uparrow \downarrow \ldots \uparrow \downarrow\rangle$ path. Note that we have suppressed the color index in this treatment since, as mentioned above, in the ground state superposition all admissible colorings should appear with the same amplitude.

The amplitude of a given Dyck path in the ground state of the model is thus given by counting the number of "diamonds" associated with each $t_{j}^{A}$, and can be written 


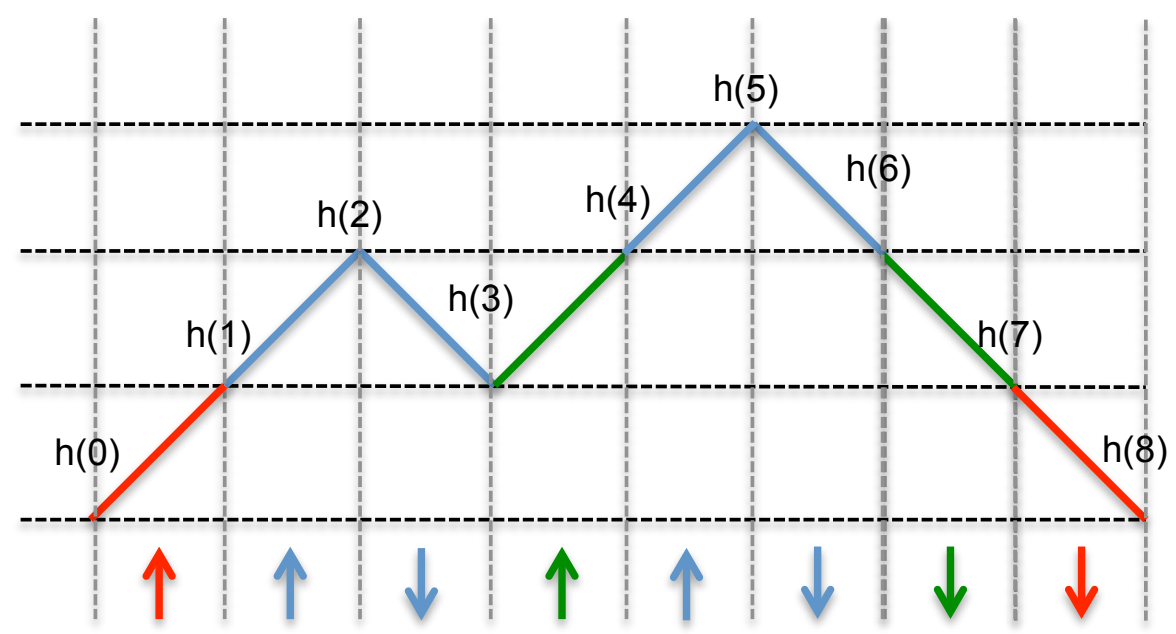

Figure 3.2: A spin configuration corresponding to a colored Dyck path and the corresponding height function $h(l)$.

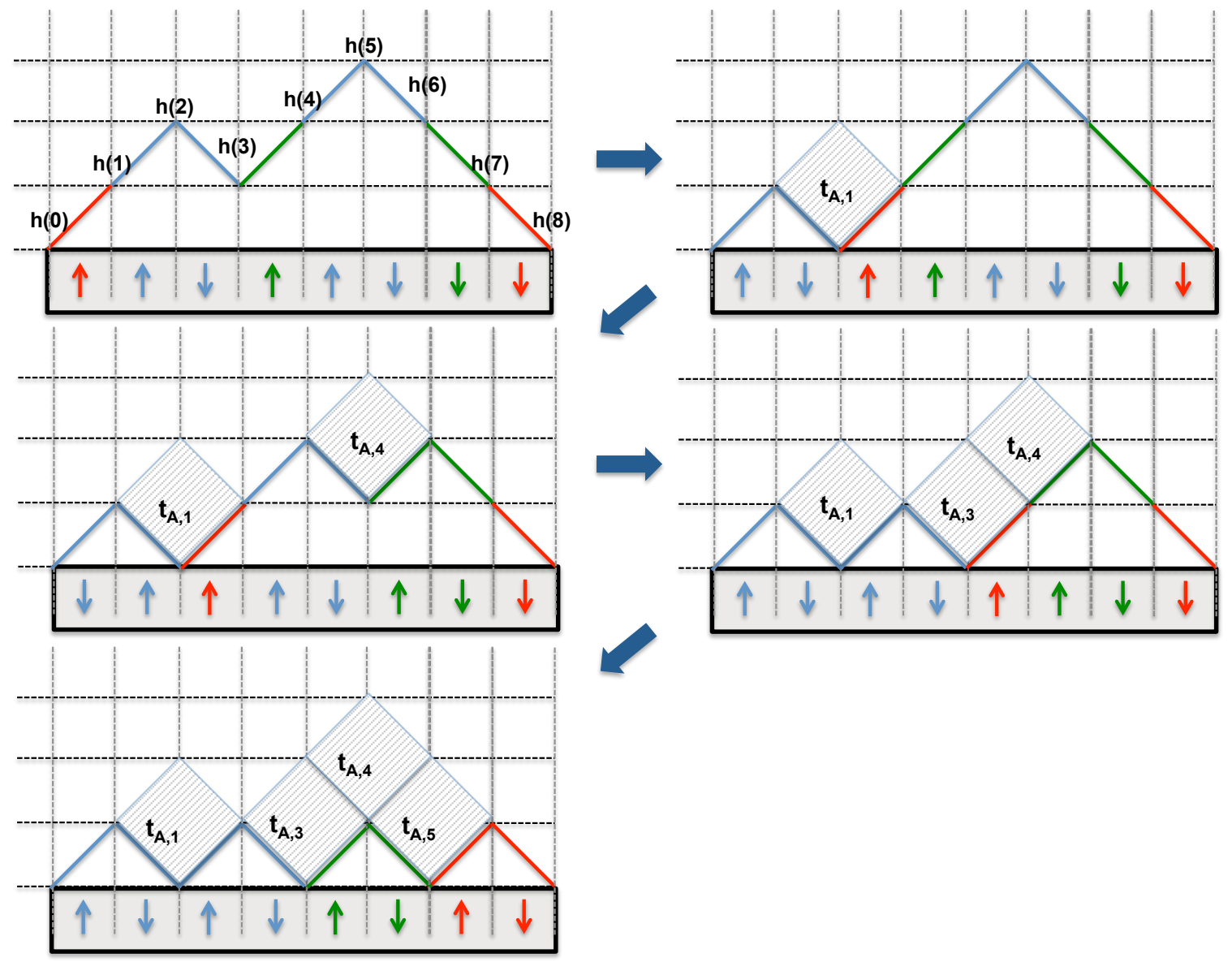

Figure 3.3: The "flattening" of a hill and it's amplitude. Starting from the left, we reduce the first by using the Fredkin move $|\uparrow \uparrow \downarrow\rangle \longrightarrow t_{A}|\uparrow \downarrow \uparrow\rangle$. This process is repeated for each peak until the lowest height hill is achieved. 
in the form:

$$
|G S\rangle=\frac{1}{\mathcal{N}} \sum_{\substack{w \in\{s-\text { colored } \\ \text { Dyck walks }\}}} e^{\sum_{l=2}^{2 n-2}\left[\frac{h(l)}{2}\right] \log \left(t_{l-1}^{A}\right)}|w\rangle
$$

where $[x]$ is the integer part of $x$ and $\mathcal{N}$ is a normalization factor. In the case where $t_{j}^{A} \equiv t$, the ground state can be simply related to the area under the path as:

$$
|G S\rangle=\frac{1}{\mathcal{N}^{\prime}} \sum_{\substack{w \in\{s-\text { colored } \\ \text { Dyck walks }\}}} t^{\frac{1}{2} \mathcal{A}(w)}|w\rangle
$$

\subsection{Entanglement entropy}

In this section, we employ the simplest choice of parameters which is translational invariant $t_{A}=t_{B}=t$ everywhere. When $t=1$, the entanglement entropy of the ground state scales as $\log n$ for $s=1$ and as $\sqrt{n}$ for $s>1$ [24, 104]. The reason our deformation with the extra parameter can further increase the scaling of entropy is because when a spin is moved around its neighboring $\uparrow \downarrow$ pair, it is separated from its own partner paired in the same color, which is the first unpaired down spin to its right (or up spin to its left). This way, when a pair of spins required to be in the same color are shifted to different subsystems of the chain, they become a source of entanglement entropy between the two subsystems. Tuning the parameter $t$ to favor higher paths in the ground state superposition will now enhance the more substantial contribution from those with more unpaired spins in one subsystem. To put this in a mathematical way, we decompose the ground state into tensor products of states in the left and right halves of the chain.

$$
|G S\rangle=\sum_{m=0}^{n} \sqrt{p_{n, m}} \sum_{x \in\left\{\uparrow^{1}, \uparrow^{2}, \ldots, \uparrow^{s}\right\}^{m}}\left|\hat{C}_{0, m, x}\right\rangle_{1, \ldots, n} \otimes\left|\hat{C}_{m, 0, \bar{x}}\right\rangle_{n+1, \ldots, 2 n}
$$


where $\left|\hat{C}_{p, q, x}\right\rangle$ is a weighted superposition of spin configurations with $p$ excess $\downarrow, q$ excess $\uparrow$ and a particular coloring $x$ of the unmatched arrows, such that $\left\langle G S\left|\left(\left|\hat{C}_{0, m, x}\right\rangle_{1, \ldots, n} \otimes\left|\hat{C}_{m, 0, \bar{x}}\right\rangle_{n+1, \ldots, 2 n}\right) \neq 0\right.\right.$, and $\bar{x}$ is the coloring in the second half of the chain that matches $x$. The decomposition gives the Schmidt number

$$
p_{n, m}(s, t)=\frac{M_{n, m}^{2}(s, t)}{N_{n}(s, t)},
$$

where

$$
\begin{aligned}
M_{n, m}(s, t) & \equiv s^{\frac{n-m}{2}} \sum_{\begin{array}{c}
w \in\{1 \text { st half of Dyck } \\
\text { walks stopping at }(n, m)\}
\end{array}} t^{\mathcal{A}(w)}, \\
N_{n}(s, t) & \equiv \sum_{m=0}^{n} s^{m} M_{n, m}^{2}(s, t) .
\end{aligned}
$$

And the entanglement entropy of the half chain in the ground state is given by

$$
S_{n}(s, t)=-\sum_{m=0}^{n} s^{m} p_{n, m}(s, t) \log p_{n, m}(s, t) .
$$

To study the behavior of $M_{n, m}$ as a function of $m$, we observe that they satisfy the following recurrence relations,

$$
\begin{aligned}
M_{k+1, k+1} & =t^{k+\frac{1}{2}} M_{k, k}, \\
M_{k+1, m} & =s t^{m+\frac{1}{2}} M_{k, m+1}+t^{m-\frac{1}{2}} M_{k, m-1}, \quad \text { for } 0<m<k, \\
M_{k+1,0} & =s t^{\frac{1}{2}} M_{k, 1} .
\end{aligned}
$$

Notice that the $M_{n, m}$ is only non-vanishing for $m$ 's of same parity as $n$.

From these relations, we can see that for large enough $t, M_{n, m}$ will be monotonically increasing as we increase $m$ by increments of 2 . Paths with height in the middle scaling as $O(n)$ will contribute more to the entanglement entropy from the $s^{O(n)}$ possible colorings of unmatched spins. In particular, the half chain entanglement entropy 
will also scale linearly with system size $n$. In the next subsection, we give a rigorous proof that this is true in the thermodynamic, and that this critical phase of large entanglement spans the entire half line $t>1$.

\subsection{1 $t>1, s>1$ phase: Volume scaling of entropy.}

In this section we repeat the steps taken in the previous chapter to prove volume scaling for weighted Motzkin walks, with a few modifications. For arbitrary $t>1$, the non-zero entries of $M_{n, m}$ are not necessarily monotonic in terms of $m$, but we can still show that for a given $n, M_{n, m}$ reaches its maximum at some $m=m^{*}$, within a finite distance away from $m=n$ independent of the system size $n$ itself. This is not obvious in the step-by-step recurrence relations, but becomes clear as we take into account the accumulated effect of the evolution of the coefficients $M_{n, m}$ with respect to $n$. To see this, we summarize (3.16) in the following operator formalism.

As in the previous chapter, we represent the distributions of $M_{k, m}$ as components of the state at 'time' $k$ during the 'evolution' in a basis spanned by $|m\rangle, m=0,1,2, \ldots$.

$$
\left|\mathcal{M}_{k}\right\rangle=\sum_{m=0}^{\infty} M_{k, m}|m\rangle, \quad M_{k, m}=0 \text { if } m>k
$$

We we define 'shift' and 'height' operators to describe the 'evolution' of the the states $\left|\mathcal{M}_{k}\right\rangle$ as

$$
\begin{aligned}
& \mathcal{S}|m\rangle=|m-1\rangle, \mathcal{S}|0\rangle=0 ; \\
& \mathcal{H}|m\rangle=m|m\rangle .
\end{aligned}
$$


One can check that the recurrence relations (3.16) translate to

$$
\begin{aligned}
& \left\langle m \mid \mathcal{M}_{k+1}\right\rangle=M_{k+1, m}=s t^{m+\frac{1}{2}}\left\langle m+1 \mid \mathcal{M}_{k}\right\rangle+t^{m-\frac{1}{2}}\left\langle m-1 \mid \mathcal{M}_{k}\right\rangle \\
= & \left\langle m\left|s t^{\mathcal{H}+\frac{1}{2}} \mathcal{S}+t^{\mathcal{H}-\frac{1}{2}} \mathcal{S}^{\dagger}\right| \mathcal{M}_{k}\right\rangle,
\end{aligned}
$$

which gives us:

$$
\left|\mathcal{M}_{k+1}\right\rangle=t^{\mathcal{H}}\left(s \sqrt{t} \mathcal{S}+\frac{1}{\sqrt{t}} \mathcal{S}^{\dagger}\right)\left|\mathcal{M}_{k}\right\rangle
$$

Using the commutation relation

$$
t^{k \mathcal{H}}\left(s \sqrt{t} \mathcal{S}+\frac{1}{\sqrt{t}} \mathcal{S}^{\dagger}\right)=\left(s t^{-\left(k-\frac{1}{2}\right)} \mathcal{S}+t^{k-\frac{1}{2}} \mathcal{S}^{\dagger}\right) t^{k \mathcal{H}}
$$

we keep moving the $t^{k \mathcal{H}}$ operators all the way to the right until it disappears when acting on $|0\rangle$ we obtain:

$$
\left|\mathcal{M}_{n}\right\rangle=\left[t^{\mathcal{H}}\left(s \sqrt{t} \mathcal{S}+\frac{1}{\sqrt{t}} \mathcal{S}^{\dagger}\right)\right]^{n}\left|\mathcal{M}_{0}\right\rangle=\overrightarrow{\mathcal{K}} \prod_{k=1}^{n}\left(s t^{-\left(k-\frac{1}{2}\right)} \mathcal{S}+t^{k-\frac{1}{2}} \mathcal{S}^{\dagger}\right)|0\rangle
$$

Here $\overrightarrow{\mathcal{K}}$ denotes ordering the multiplications in the product such that factors with greater $k$ value are on the right. For $t>1$ the factors in the product above are dominated by the $\mathcal{S}^{\dagger}$ term for large $k$. In other words, at some point during the evolution, the distribution of $M_{m}$ starts shifting at velocity 1 to the right along the $m$ axis without much spreading. For a larger $t$, this happens shortly after the evolution starts, while for smaller values of $t$, it takes longer to reach this stable propagation. In any case, as we show below, the maximum of $M_{n, m}$ is a within finite distance away from $m=n$.

Lemma III.1. Let $m^{*}$ be such that $\sup _{m} M_{n, m}=M_{n, m^{*}}$, then $\exists N_{0}<n$, such that when $t>1, m^{*} \in\left[n-2 N_{0}, n\right]$. 
Proof. Let

$$
\left|\mathcal{M}_{n}^{\prime}\right\rangle=\overrightarrow{\mathcal{K}} \prod_{k=N_{0}+1}^{n}\left(s t^{-\left(k-\frac{1}{2}\right)} \mathcal{S}+t^{k-\frac{1}{2}} \mathcal{S}^{\dagger}\right)|0\rangle
$$

Note that

$$
t^{-\left(k-\frac{1}{2}\right)}\left\|s t^{-\left(k-\frac{1}{2}\right)} \mathcal{S}\right\|_{1} \leq s t^{-2\left(k-\frac{1}{2}\right)} \equiv c_{k},
$$

so that:

$$
\left\|s t^{-\left(k-\frac{1}{2}\right)} \mathcal{S}+t^{k-\frac{1}{2}} \mathcal{S}^{\dagger}\right\|_{1}<t^{k-\frac{1}{2}}\left(1+c_{k}\right)
$$

we thus have

$$
\begin{gathered}
\| t^{-\sum_{k=N_{0}+1}^{n}\left(k-\frac{1}{2}\right)}\left|\mathcal{M}_{n}^{\prime}\right\rangle-\left|n-N_{0}\right\rangle \|_{1} \leq \prod_{k=N_{0}+1}^{n}\left(c_{k}+1\right)-1<e^{\sum_{k=N_{0}+1}^{\infty} c_{k}}-1 \\
=e^{\frac{s t}{t^{2}-1} t^{-2 N_{0}}}-1 \equiv f(s, t)^{t^{-2 N_{0}}}-1 .
\end{gathered}
$$

The first inequality on the left follows from noting that $\left|n-N_{0}\right\rangle$ appears in $t^{-\sum_{k=N_{0}+1}^{n}\left(k-\frac{1}{2}\right)}\left|\mathcal{M}_{n}^{\prime}\right\rangle$ with coefficient 1 , and is exactly canceled. We have also used that $x+1 \leq e^{x}$. Next,

$$
\begin{aligned}
& \| t^{-\sum_{k=1}^{n}\left(k-\frac{1}{2}\right)}\left|\mathcal{M}_{n}\right\rangle-\overrightarrow{\mathcal{K}} \prod_{k=1}^{N_{0}}\left(s t^{-2\left(k-\frac{1}{2}\right)} \mathcal{S}+\mathcal{S}^{\dagger}\right)\left|n-N_{0}\right\rangle \|_{1} \\
\leq & \left\|\overrightarrow{\mathcal{K}} \prod_{k=1}^{N_{0}}\left(s t^{-2\left(k-\frac{1}{2}\right)} \mathcal{S}+\mathcal{S}^{\dagger}\right)\right\|_{1} \| t^{-\sum_{k=N_{0}+1}^{n}\left(k-\frac{1}{2}\right)}\left|\mathcal{M}_{n}^{\prime}\right\rangle-\left|n-N_{0}\right\rangle \|_{1} \\
< & \left(f(s, t)^{t^{-N_{0}}}-1\right) \prod_{k=1}^{N_{0}}\left(1+c_{k}\right)<\left(f(s, t)^{t^{-N_{0}}}-1\right) e^{\sum_{k=1}^{N_{0}} c_{k}}<\left(f(s, t)^{t^{-N_{0}}}-1\right) f(s, t) .
\end{aligned}
$$

Let

$$
M_{n, m}^{\prime}=\left\langle m\left|\overrightarrow{\mathcal{K}} \prod_{k=1}^{N_{0}}\left(s t^{-2\left(k-\frac{1}{2}\right)} \mathcal{S}+\mathcal{S}^{\dagger}\right)\right| n-N_{0}\right\rangle
$$


then clearly $M_{n, m}^{\prime}=0$ for $m<n-2 N_{0}$. If we choose

$$
N_{0}= \begin{cases}0 & f(s, t)<\frac{1+\sqrt{5}}{2}, \\ -\frac{\log \frac{\log \left(f^{-1}(s, t)+1\right)}{\log f(s, t)},}{\log t}, & \text { otherwise }\end{cases}
$$

then

$$
\| t^{-\frac{n^{2}}{2}}\left|\mathcal{M}_{n}\right\rangle-\sum_{m=n-2 N_{0}}^{n} M_{n, m}^{\prime}|m\rangle \|_{1}<1=M_{n, n}^{\prime} \leq \sup _{m} M_{n, m}^{\prime} .
$$

Therefore $\exists m^{*} \in\left[n-2 N_{0}, n\right]$, such that $M_{n, m^{*}} \geq M_{n, m}$ for all $m$.

This allows us to prove the linear scaling of the entanglement entropy.

Theorem III.1. In the state (3.10), when $t>1$, the entanglement entropy of half of the chain is bounded from below by $S_{n}>n \log s+C(s, t)$, where $C(s, t)$ is an $n$ independent constant.

Proof. We separate a linear term from $S_{n}$ as follows (below we supress the $n$ index in $\left.M_{n, m}\right)$ :

$$
\begin{aligned}
S_{n} & =\sum_{m=0}^{n} s^{m} p_{m} \log s^{m}-\sum_{m=0}^{n} s^{m} p_{m} \log \left(s^{m} p_{m}\right)>\sum_{m=0}^{n} s^{m} p_{m} m \log s \\
& =\sum_{l=0}^{n} s^{n-l} p_{n-l}(n-l) \log s=n \log s-\log s \sum_{l=0}^{n} \frac{s^{n-l} M_{n-l}^{2}}{\sum_{m^{\prime}=0}^{n} s^{m^{\prime}} M_{m^{\prime}}^{2}} l
\end{aligned}
$$

Taking $m^{*}$ such that $\sup _{m} M_{n, m}=M_{n, m^{*}}$ and using lemma III.1, we see that

$$
\begin{aligned}
& \sum_{l=0}^{n} \frac{s^{n-l} M_{m^{*}}^{2}}{\sum_{m^{\prime}=0}^{n} s^{m^{\prime}} M_{m^{\prime}}^{2}} l<\sum_{l=0}^{n} \frac{s^{n-l} M_{m^{*}}^{2}}{s^{m^{*}} M_{m^{*}}^{2}} l=s^{n-m^{*}} \sum_{l=0}^{n} s^{-l} l \\
& <s^{2 N_{0}} \sum_{l=0}^{n} s^{-l} l<s^{2 N_{0}} \sum_{l=0}^{\infty} s^{-l} l=\frac{s^{2 N_{0}+1}}{(s-1)^{2}} .
\end{aligned}
$$

Therefore, the remainder term on the right hand side of (3.28) is bounded. 
One can see from the proof that the factor of $s^{m}$ is already enough to make the scaling of entropy linear, and all that is required for $p_{m}$ is that it doesn't destroy this exponential dependence on $m$.

\subsection{2 $t<1$ and any $s:$ Bounded entanglement entropy.}

Contrary to the case studied above, when $t<1$, we expect Dyck paths with smaller areas below to be exponentially preferred in the ground state superposition. But this time, for the entropy to reflect the predominance of lower path, where less mutual information between the two subsystems can be stored, the behavior of $p_{m}$ needs to not only be decreasing exponentially with $m$, but also fast enough to overcome the exponential increasing $s^{m}$ factor. Considering that, we define

$$
\tilde{M}_{n, m}=s^{\frac{m}{2}} M_{n, m}, \tilde{p}_{n, m}=\frac{\tilde{M}_{n, m}^{2}}{\sum_{m=0}^{n} \tilde{M}_{n, m}^{2}} .
$$

Substitution into (3.16) gives the following relations,

$$
\begin{aligned}
\tilde{M}_{k+1, k+1} & =\sqrt{s} t^{k+\frac{1}{2}} \tilde{M}_{k, k}, \\
\tilde{M}_{k+1, m} & =\sqrt{s}\left(t^{m+\frac{1}{2}} \tilde{M}_{k, m+1}+t^{m-\frac{1}{2}} \tilde{M}_{k, m-1}\right), \quad 0<m<k, \\
\tilde{M}_{k+1,0} & =\sqrt{s} t^{\frac{1}{2}} \tilde{M}_{k, 1}
\end{aligned}
$$

To prove the entropy is bounded, we need the following lemmas.

\section{Lemma III.2.}

$$
\tilde{M}_{n+2, m}^{2}>\tilde{M}_{n, m}^{2}
$$


Proof. From (3.22), we have

$$
\begin{aligned}
\left|\mathcal{M}_{n+2}\right\rangle & =\overrightarrow{\mathcal{K}} \prod_{k=1}^{n}\left(s t^{-\left(k-\frac{1}{2}\right)} \mathcal{S}+t^{k-\frac{1}{2}} \mathcal{S}^{\dagger}\right)\left(s t^{-\left(n+\frac{1}{2}\right)} \mathcal{S}+t^{n+\frac{1}{2}} \mathcal{S}^{\dagger}\right)\left(s t^{-\left(n+\frac{3}{2}\right)} \mathcal{S}+t^{n+\frac{3}{2}} \mathcal{S}^{\dagger}\right)|0\rangle \\
& =\overrightarrow{\mathcal{K}} \prod_{k=1}^{n}\left(s t^{-\left(k-\frac{1}{2}\right)} \mathcal{S}+t^{k-\frac{1}{2}} \mathcal{S}^{\dagger}\right)\left[s^{2} t^{-2(n+1)} \mathcal{S}^{2}+s\left(t+\frac{1}{t}\right)+t^{2(n+1)} \mathcal{S}^{\dagger 2}\right]|0\rangle \\
& =s\left(t+\frac{1}{t}\right)\left|\mathcal{M}_{n}\right\rangle+\overrightarrow{\mathcal{K}} \prod_{k=1}^{n}\left(s t^{-\left(k-\frac{1}{2}\right)} \mathcal{S}+t^{k-\frac{1}{2}} \mathcal{S}^{\dagger}\right)\left[s^{2} t^{-2(n+1)} \mathcal{S}^{2}+t^{2(n+1)} \mathcal{S}^{\dagger 2}\right]|0\rangle .
\end{aligned}
$$

The last term on the RHS of the equation contains non-zero contributions for all states $|m\rangle$, with $m=0,1, \ldots, n+2$, and we have:

$$
\begin{aligned}
& M_{n+2, m}>M_{n, m}, \\
& \tilde{M}_{n+2, m}>\tilde{M}_{n, m} \quad \forall m \geq 0, n \geq 1 .
\end{aligned}
$$

Next we establish the following bound on $\tilde{p}_{n, m}$ :

\section{Lemma III.3.}

$$
\tilde{p}_{n, m}<36 \frac{s^{2}}{t^{2}} t^{4 m}
$$

Proof. By definition of $\tilde{p}_{n, m}$, and using the recursion relation (3.30) twice consequtively,

$$
\begin{aligned}
\tilde{p}_{n, m} & =\frac{s^{2} t^{4 m}\left[t^{2} \tilde{M}_{n-2, m+2}+\left(t+\frac{1}{t}\right) \tilde{M}_{n-2, m}+t^{-2} \tilde{M}_{n-2, m-2}\right]^{2}}{\sum_{m=0}^{n} \tilde{M}_{n, m}^{2}} \\
& \leq \frac{s^{2} t^{4 m}\left[3 \max \left\{t^{2} \tilde{M}_{n-2, m+2},\left(t+\frac{1}{t}\right) \tilde{M}_{n-2, m}, t^{-2} \tilde{M}_{n-2, m-2}\right\}\right]^{2}}{\sum_{m=0}^{n} \tilde{M}_{n, m}^{2}} \\
& \leq 36 \frac{s^{2}}{t^{2}} t^{4 m} \frac{\max \left\{\tilde{M}_{n, m+2}^{2}, \tilde{M}_{n, m}^{2}, \tilde{M}_{n, m-2}^{2}\right\}}{\sum_{m=0}^{n} \tilde{M}_{n, m}^{2}}<36 \frac{s^{2}}{t^{2}} t^{4 m} .
\end{aligned}
$$

Lemma III.2 was used in the last line. 
We now have the ingredients to prove the boundedness of entropy.

Theorem III.2. When $0<t<1, s \geq 1$, there exists a constant $C_{0}(s, t)$ independent of the system size $n$, that for any $n, S_{n}<C_{0}(s, t)$.

Proof. Using Lemma III.3 we see that when

$$
m>m_{0} \equiv\left[\frac{\log \left(\frac{1}{36 e} \frac{t^{2}}{s^{2}}\right)}{4 \log t}\right]+1,
$$

we have

$$
\tilde{p}_{n, m}<36 \frac{s^{2}}{t^{2}} t^{4 m}<\frac{1}{e}
$$

It is easy to check that the function $-x \log (x)$ is monotonically increasing when $x \in\left(0, \frac{1}{e}\right)$, in other words, for $m>m_{0}$,

$$
\tilde{p}_{n, m}<36 \frac{s^{2}}{t^{2}} t^{4 m}<\frac{1}{e} \Longrightarrow-\tilde{p}_{n, m} \log \tilde{p}_{n, m}<-36 \frac{s^{2}}{t^{2}} t^{4 m}\left(\log \left(\frac{36 s^{2}}{t^{2}}\right)+4 m \log t\right)
$$

Therefore

$$
\begin{aligned}
S_{n}= & -\sum_{m=0}^{n} \tilde{p}_{n, m} \log \tilde{p}_{n, m}+\log s \sum_{m=0}^{n} \tilde{p}_{n, m} m \\
< & -\sum_{m=0}^{m_{0}} \tilde{p}_{n, m} \log \tilde{p}_{n, m}-\sum_{m=m_{0}+1}^{\infty} \frac{36 s^{2}}{t^{2}} t^{4 m}\left(\log \left(\frac{36 s^{2}}{t^{2}}\right)+4 m \log t\right) \\
& +\log s \sum_{m=0}^{\infty} \frac{36 s^{2}}{t^{2}} t^{4 m} m \\
< & \frac{m_{0}+1}{e}-\frac{36 s^{2} t^{4 m_{0}+2}}{1-t^{4}} \log \left(\frac{36 s^{2}}{t^{2}}\right)-\frac{144 s^{2} t^{4 m_{0}+2}\left(m_{0}\left(1-t^{4}\right)+1\right)}{\left(t^{4}-1\right)^{2}} \log t \\
& +\frac{36 s^{2} t^{2}}{\left(t^{4}-1\right)^{2}} \log s \\
\equiv & C_{0}(s, t),
\end{aligned}
$$

where we used $\sup _{x \in(0,1)}-x \log (x)=e^{-1}$ for entropy terms with $m \leq m_{0}$ in the last inequality. 
Notice our proof here does not rely on the fact that $s>1$, and it applies to the $s=1$ case as well.

\subsection{Scaling of the Spectral Gap}

\subsubsection{Super-exponential Upper bound in the $t>1, s=1$ Phase}

Since entanglement entropy is a measure of correlation in the system, a high entanglement entropy indicates that the system is highly correlated and also a gapless spectrum (in the thermodynamic limit) $[48,8]$. As our model at $t>1, s>1$ exhibits linear scaling of entanglement entropy, we expect the spectral gap to be also decreasing faster with system size than the $t=1$. Here, we give variational proof that the spectral gap for $t>1, s>1$ decreases exponentially with a square of the system size.

Just as the linear scaling of entanglement entropy results from the prominence of the higher weighted paths in the ground state superposition, gaplessness can be shown by truncating lower weighted paths at the price of softly violating the superposition required to make the projectors in the Hamiltonian vanishing. To do so it is convenient to define a 'prime walk' as follows:

Definition III.1. A prime Dyck walk is a Dyck walk that is always above the $x$-axis, except at the endpoints.

By this definition, a Dyck walk is either prime or a concatenation of prime walks (Fig. 3.4 exhibits a Dyck walk in solid line made of two prime walks and one in dashed line made of three prime walks).

To construct a low energy variational excited state, we start with an auxiliary state that projects out all the walks in the ground state superposition whose longest prime walk has a length smaller than $n+1$. That is, define:

$$
P_{n,>}=\{\text { s-colored walks containing a prime walk of length } l>n\},
$$


and $P_{n,<}=$ the complement of $P_{n,>}$. Our auxiliary state is defined as:

$$
\frac{1}{\mathcal{N}^{*}} \sum_{w \in P_{n,>}} t^{\frac{1}{2} \mathcal{A}(w)}|w\rangle
$$

For $t>1$ higher walks are favored rendering the auxiliary state largely overlapping with the ground state and therefore unqualified as a low energy excitation state. However, the color degree of freedom allows us to make this state orthogonal to the ground state by permuting the color of the last down move (or equivalently the first up move) in the longest prime walk. This way, all walks in the new superposition have one pair of spins with unmatched colors, and consequently orthogonal to all paths in the ground state. The choice of the ' $n+1$ ' threshold on the cut-off of longest prime walk length eliminates the potential ambiguity in the location of the color permutation so that each path in the superposition has exactly one pair of unmatched colores.

Theorem III.3. The spectral gap of the $t>1, s>1$ phase has an upper bound of $\frac{2(4 s)^{n}}{1+t^{2}} t^{-n^{2} / 2}$.

Proof. We define a new state $|\xi\rangle$ as:

$$
|\xi\rangle=\frac{1}{\mathcal{N}^{*}} \sum_{w \in P_{n,>}} t^{\frac{1}{2} \mathcal{A}(w)} \mathcal{P}|w\rangle,
$$

where $\mathcal{N}^{*}$ is the new normalization factor and the operator $\mathcal{P}$ sends the color $c$ of the last down move of the longest prime walk to $c+1 \bmod s$ and leaves everything else unchanged. Because of the color imbalance we immediately have:

$$
\langle\xi \mid G S\rangle=0
$$

and $|\xi\rangle$ can be readily used as a variational wave function to bound the gap from above . 


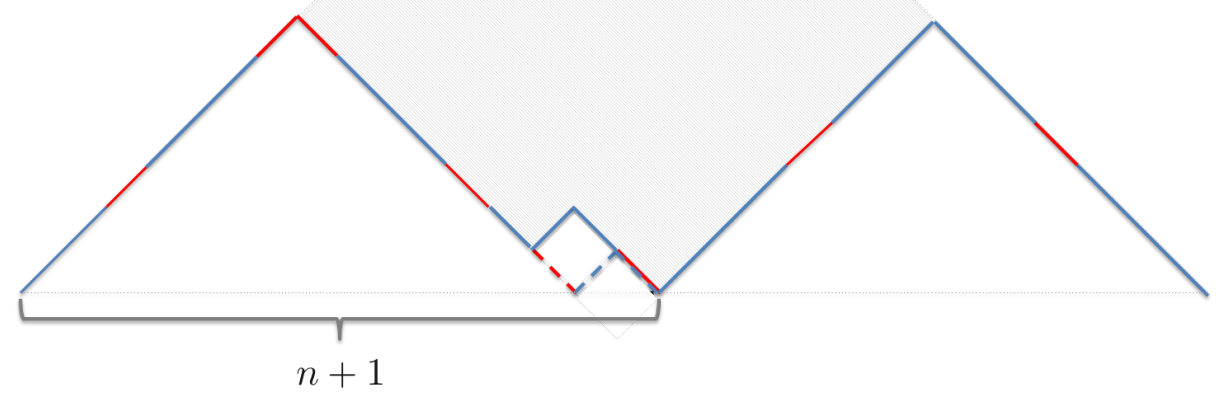

Figure 3.4: An representative walk in the superposition of $|\xi\rangle$ that crosses the threshold of the cut-off when acted on by the operator $\left(t\left|\downarrow_{n-1}^{r} \uparrow_{n}^{b} \downarrow_{n+1}^{b}\right\rangle-\right.$ $\left.\left|\uparrow_{n-1}^{b} \downarrow_{n}^{b} \downarrow_{n+1}^{r}\right\rangle\right)\left(\left\langle\downarrow_{n-1}^{r} \uparrow_{n}^{b} \downarrow_{n+1}^{b}\right| t-\left\langle\uparrow_{n-1}^{b} \downarrow_{n}^{b} \downarrow_{n+1}^{r}\right|\right)$.

Let us compute the variational energy associated with the $|\xi\rangle$ state. First we note that:

$$
H_{\partial}|\xi\rangle=0, H_{X}|\xi\rangle=0
$$

as each non-matching color pair is separated by at least $n$ sites (while $H_{X}$ is only sensitive to nearest neighbor violations). The same goes for most of the projectors in $H_{F}$ just the way it works in the ground state.

However, in $H_{F}$, we have also non-zero contributions coming from walks $w$ that are one "Fredkin" move away from leaving the set $P_{n},>$. In other words, this happens when the first (second) projector in $H_{F}$ in Eq. (3.1) acts on the left (resp. right) endpoint of the longest prime walk and changes its length from $n+1$ to $n-1$ (the kind of which is absent in the superposition). For instance, applying the projectors on $\phi_{n-1, B}($ Eq. (3.7)) to the prime walk $w$ corresponding to the one in Fig. 3.4 gives: 
$\frac{1}{1+t^{2}}\left\langle w\left|\left(t\left|\downarrow_{n-1}^{r} \uparrow_{n}^{b} \downarrow_{n+1}^{b}\right\rangle-\left|\uparrow_{n-1}^{b} \downarrow_{n}^{b} \downarrow_{n+1}^{r}\right\rangle\right)\left(\left\langle\downarrow_{n-1}^{r} \uparrow_{n}^{b} \downarrow_{n+1}^{b}\left|t-\left\langle\uparrow_{n-1}^{b} \downarrow_{n}^{b} \downarrow_{n+1}^{r}\right|\right) \mid w\right\rangle=\frac{1}{1+t^{2}}\right.\right.\right.$,

and

$$
\frac{1}{1+t^{2}}\left\langlew ^ { \prime } \left|( t | \downarrow _ { n - 1 } ^ { r } \uparrow _ { n } ^ { b } \downarrow _ { n + 1 } ^ { b } \rangle - | \uparrow _ { n - 1 } ^ { b } \downarrow _ { n } ^ { b } \downarrow _ { n + 1 } ^ { r } \rangle ) \left(\left\langle\downarrow_{n-1}^{r} \uparrow_{n}^{b} \downarrow_{n+1}^{b}\left|t-\left\langle\uparrow_{n-1}^{b} \downarrow_{n}^{b} \downarrow_{n+1}^{r}\right|\right) \mid w\right\rangle=0,\right.\right.\right.
$$

with $w^{\prime}$ is any other walk in the $|\xi\rangle$ (i.e. any other walk in $P_{n,>}$ ).

We can now estimate the variational energy due to such paths. The number of these paths that will go from $P_{n,>}$ to $P_{n,<}$ when applying a Fredkin projector is very roughly bounded from above by $2^{2 n} s^{n}$ (which is the total number of walks). On the other hand, the probability amplitudes of a path that has a prime walk length of exactly $n+1$ or $n+2$ in $P_{n,<}$, are penalized by their area differences from the highest weighted one, i.e. the shaded area in Fig. 3.4, by a factor smaller than $t^{-n^{2} / 4}$. We therefore have the following upper bound:

$$
\langle\xi|H| \xi\rangle<\frac{2(4 s)^{n}}{1+t^{2}} t^{-n^{2} / 2}
$$

Thus we have proved an upper bound of exponential of square of system size on the spectral gap when $t>1, s>1$.

Remark: The overall factor 2 above comes from possibility of modifying the prime path on the left or on the right.

\subsubsection{Exponential Upper Bound in the $t>1, s=1$ Phase}

As has been discussed in the previous subsection, a bounded from above entanglement entropy is expected to be a strong indicator of the existence of a non-vanishing spectral gap. Yet that intuition fails in the $t>1, s=1$ phase of the Motzkin spin 


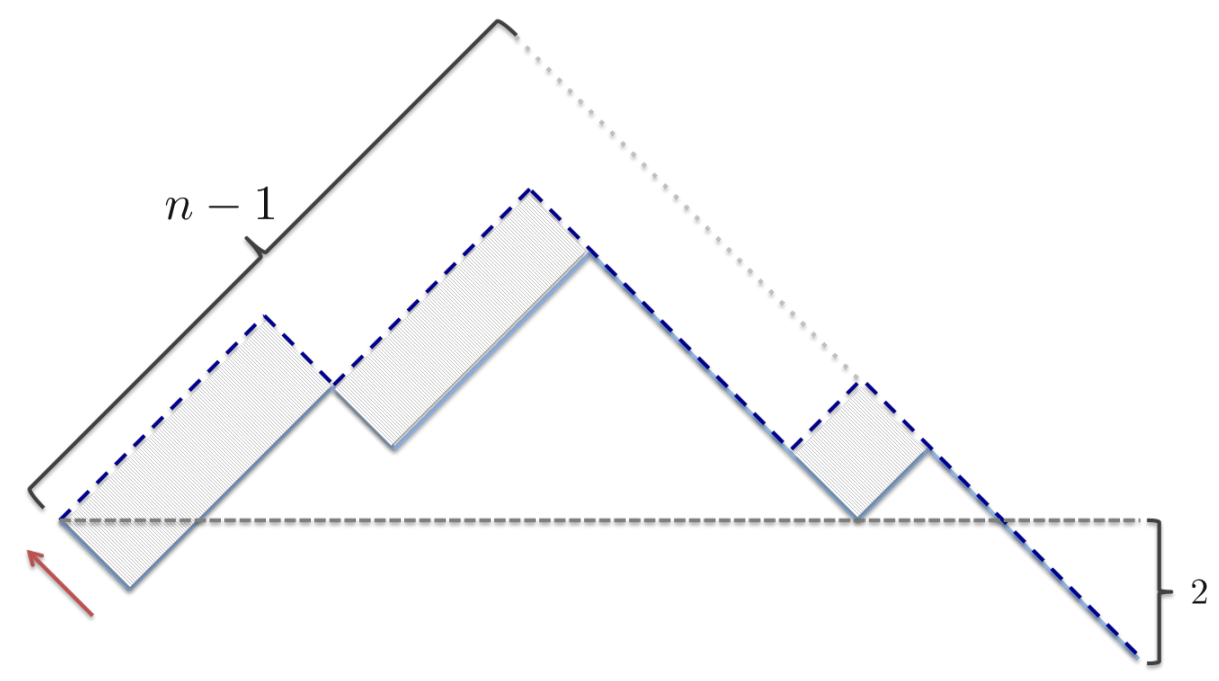

Figure 3.5: Two representative walks in $Q_{-2}$. The light blue one can be shifted in the direction of the orange arrow to become the dark blue one with a relative weight increase of $t^{n-1}$ corresponding to the area of the shaded regions.

chain. The numerical results in [12] showed the $t>1, s=1$ Motzkin chain is gapless despite the boundedness of its entanglement entropy. Here we prove the Fredkin chain counterpart of this phenomenon, which can be readily adapted to the Motzkin chain.

We follow the same strategy we used to construct low energy excitation state from the $t>1, s>1$ phase, only now we don't have the luxury of taking advantage of color degrees of freedom to ensure the orthogonality to the ground state. Fortunately, there's still a degree of freedom we haven't fully exploited yet, namely the z-component of the total spin, or the net up spin of the chain, which can be nonvanishing when the boundary terms in the Hamiltonian is violated. To construct a low energy excitation due to this, we define

$Q_{-2}=\{$ walks that starts from $(0,0)$, ends at $(2 \mathrm{n},-2)$ and never pass below $\mathrm{x}=-2$.

Notice a Fredkin move acting on a walk in $Q_{-2}$ always gives another walk in $Q_{-2}$.

Theorem III.4. The spectral gap of the $t>1, s=1$ phase has an upper bound of $t^{-n+1}$ 
Proof. We define an excited state

$$
|\eta\rangle=\frac{1}{N^{\star}} \sum_{w \in Q_{-2}} t^{\frac{1}{2} A(w)}|w\rangle,
$$

where $N^{\star}=\left(\sum_{w \in Q_{-2}} t^{A(w)}\right)^{\frac{1}{2}}$ is the normalization factor. $|\eta\rangle$ is clearly orthogonal to the ground state as they have different total spins. Since $|\eta\rangle$ only violates the boundary term in the Hamiltonian, after being acted on by $H$, only paths starting with a down move will survive. To get an estimate on the amplitude of the paths left, we point out that by rearranging the first down step to the last, (or equivalently shifting along the arrow in Fig. 3.5,) we get another walk in $Q_{-2}$ of area $2 n-2$ bigger. Therefore,

$$
\langle\eta|H| \eta\rangle=\frac{\sum_{w \in Q_{-2}} t^{A(w)}}{N^{\star}}<t^{-n+1}
$$

which gives an upper bound on the spectral gap. 


\section{Chapter IV}

\section{Coupled Wire Model of Majorana Surfaces of Topological Superconductors}

In this chapter, we introduce the single-body coupled Majorana wire model at the beginning of section 4.1. A review on the $s o(N)_{1}$ WZW CFT will be given in section 4.1.1 and 4.1.2 as well as in appendix A, B and C. In section 4.2, we will construct time reversal symmetry 4-fermion interactions that will open up an excitation energy gap. The discussion will be decomposed into the even and odd $N$ cases in section 4.2.1 and 4.2.2 respectively. In the even case, the gapping Hamiltonian will match the $O(r)$ Gross-Neveu model[43, 145, 138, 113] and we will show an energy gap in section 4.2.1.1 by (partially) bosonizing the problem. The gapping potential for the odd case will rely on a conformal embedding and relate to the Zamolodchikov and Fateev $\mathbb{Z}_{6}$ parafermion CFT[29, 144]. This will be discussed and reviewed in section 4.2.2.1, 4.2.2.2 as well as in appendix D. The symmetric gapping interactions will correspond to non-trivial surface topological orders. This will be discussed in section 4.3 where we will present the class of 32 -fold periodic topological $G_{N}$ states. In section 4.4, we will describe alternative gapping interactions that would lead to even more possibilities. 


\subsection{Coupled wire construction of surface Majorana cones}

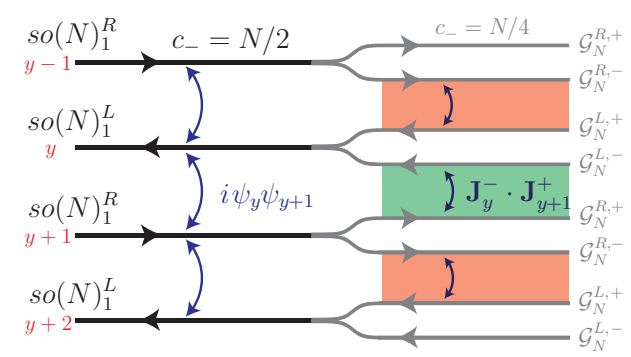

Figure 4.1: (Left) Coupled wire model (4.3) of $N$ gapless surface Majorana cones. (Right) Fractionalization (4.33) and coupled wires construction (4.37) of gapped anomalous and topological surface state.

To construct explicit many-body interactions that can remove the gapless surface degrees of freedom, we turn to an anisotropic description of surface Majorana fermions using an array of coupled fermion wires (see figure 4.1). The horizontal wires are labeled according to their vertical position $y=\ldots,-2,-1,0,1,2, \ldots$ and each carries $N$ chiral (real) Majorana fermions $\boldsymbol{\psi}_{y}=\left(\psi_{y}^{1}, \ldots, \psi_{y}^{N}\right)$ which propagate only to the right (or left) if $y$ is even (resp. odd). The number of flavors $N$ here is going to be identified with the net chirality of the surface Majorana cone. Time reversal symmetry is non-local in this model as it relates fermions on adjacent wires that propagate in opposite directions,

$$
\mathcal{T}\left(\sum_{a=1}^{N} \alpha_{a} \psi_{y}^{a}\right) \mathcal{T}^{-1}=(-1)^{y} \sum_{a=1}^{N} \alpha_{a}^{*} \psi_{y+1}^{a}
$$

Similar to the symmetry of an antiferrormagnet, here time reversal on the singlefermion Hilbert space squares to a primitive translation up to a sign, $\mathcal{T}^{2}=-\hat{t}_{y}$ for $\hat{t}_{y}$ the vertical lattice translation $y \rightarrow y+2$ that relates nearest co-propagating wires. In the many-body Hilbert space,

$$
\mathcal{T}^{2}=(-1)^{F} \hat{t}_{y}
$$


where $(-1)^{F}$ is the fermion parity operator whose sign depends on the eveness or oddness of fermion number.

We mimic $N$ copies of surface Majorana cones by the coupled wire Hamiltonian

$$
\mathcal{H}_{0}=\sum_{y=-\infty}^{\infty} i v_{\mathrm{x}}(-1)^{y} \boldsymbol{\psi}_{y}^{T} \partial_{x} \boldsymbol{\psi}_{y}+i v_{\mathrm{y}} \boldsymbol{\psi}_{y}^{T} \boldsymbol{\psi}_{y+1}
$$

where the $N$-component Majorana fermion $\boldsymbol{\psi}$ disperses linearly (for small $k_{y}$ ) with velocities $v_{\mathrm{x}}, v_{\mathrm{y}}$ along the horizontal and vertical axes (see figure 4.2). By applying (4.1), we see $\mathcal{T} \mathcal{H}_{0} \mathcal{T}^{-1}=\mathcal{H}_{0}$ and the coupled wire model is therefore time reversal symmetric. Moreover, $\mathcal{H}_{0}$ has continuous translation symmetry along $x$ and discrete translation along $y \rightarrow y+2$. The alternating sign in the first term of (4.3) specifies the propagating directions of the wires. Projecting to the $k_{x}=0$ zero modes along the wires, the second term in (4.3) effectively becomes a 1D Kitaev Majorana chain[61] which has a linear spectrum for small $k_{y}$. More explicitly, by using the Nambu basis $\boldsymbol{\xi}_{\mathbf{k}}=\left(c_{\mathbf{k}}^{a}, c_{-\mathbf{k}}^{a}\right)^{T}$ for $c_{\mathbf{k}}^{a}=\sum_{x y} e^{i\left(k_{x} x+k_{y} y\right)} c_{y}^{a}(x)$ the Fourier transform of the Dirac fermion $c_{y}^{a}(x)=\left(\psi_{2 y-1}^{a}(x)+i \psi_{2 y}^{a}(x)\right) / 2$, the coupled wire Hamiltonian (4.3) can be expressed as $\mathcal{H}_{0}=\sum_{\mathbf{k}} \boldsymbol{\xi}_{\mathbf{k}}^{\dagger} H_{\mathrm{BdG}}^{0}(\mathbf{k}) \boldsymbol{\xi}_{\mathbf{k}}$, where the BdG Hamiltonian is given by

$$
H_{\mathrm{BdG}}^{0}(\mathbf{k})=2 v_{\mathrm{x}} k_{x} \tau_{x}+v_{\mathrm{y}}\left[-\sin k_{y} \tau_{y}+\left(1-\cos k_{y}\right) \tau_{z}\right]
$$

for $-\infty<k_{x}<\infty$ and $-\pi \leq k_{y} \leq \pi$. It has a linear spectrum near zero energy and momentum as shown in figure 4.2.

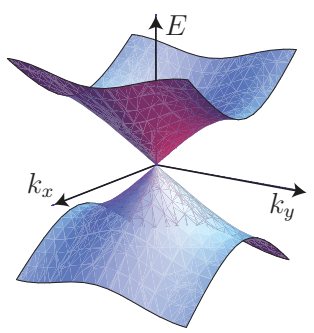

Figure 4.2: The energy spectrum of the coupled Majorana wire model (4.3) 
We notice in passing that if the time reversal operation in (4.1) was defined without the alternating sign $(-1)^{y}$, it would sqaure to a different sign $\mathcal{T}^{2}=+\hat{t}_{y}$ in the single-fermion Hilbert space and the vertical term in (4.3) would need to be modified into $\sum_{y} i v_{y}(-1)^{y} \boldsymbol{\psi}_{y}^{T} \boldsymbol{\psi}_{y+1}$ in order to preserve the symmetry. This would correspond to an alternating Majorana chain in the $y$-direction, where the gapless Majorana cone would be positioned at $k_{y}=\pi$ instead of 0 and would still be protected by Kramers theorem as $T_{k_{y}=\pi}^{2}=e^{i k_{y}}=-1$. This scenario is actually equivalent and related to the original by a gauge transformation $\left(\psi_{4 y}, \psi_{4 y+1}, \psi_{4 y+2}, \psi_{4 y+1+3}\right) \rightarrow$ $\left(\psi_{4 y}, \psi_{4 y+1},-\psi_{4 y+2},-\psi_{4 y+1+3}\right)$, and therefore the sign of $\mathcal{T}^{2}$ is unimportant in this problem. Nevertheless we will stick with previous convention defined in (4.1) in the following discussions.

The chirality $N$ of the coupled Majorana wire model (4.3) is set by the chiral central charge $c_{-}=N / 2$ along each wire. This quantity is defined by the difference of central charges[26] between right and left moving modes, and determines the energy (thermal) current $I_{T} \approx c_{-} \frac{\pi^{2} k_{B}^{2}}{6 h} T^{2}$ flowing along the wire in low temperature[54, 18, 60, 69]. In general, a Majorana wire carrying $N_{R}$ right moving fermions and $N_{L}$ left moving ones has the kinetic Hamiltonian $\mathcal{H}=i v_{\times} \boldsymbol{\psi}^{T} \not \not_{x} \boldsymbol{\psi}$, where $\not_{x}=\left[\mathbb{1}_{N_{R}} \oplus\right.$ $\left.\left(-\mathbb{1}_{N_{L}}\right)\right] \partial_{x}$ acts on the $\left(N_{R}+N_{L}\right)$-component real fermion $\boldsymbol{\psi}$. In (4.3) we consider the simplest case when $\left(N_{R}, N_{L}\right)=(N, 0)$ for $y$ even or $(0, N)$ for $y$ odd.

A chiral 1D system violates fermion doubling[87] and can only be realized as an anomalous edge of a gapped 2D bulk[126, 97, 60]. The coupled Majorana wire model, (4.3) or figure 4.1, must therefore also be holographic and living on the surface of a 3D bulk superconductor. This can be modeled by a stack of alternating layers of spinless $p_{x} \pm i p_{y}$ superconductors (see figure 4.3(a)). The interwire backscattering in (4.3) can be generated by bulk interlayer electron tunneling and pairing that are not competing with the intralayer $p+i p$ pairing. Time reversal (4.1) extends to the three dimensional bulk by relating fermions on adjacent layers. The coupled Majorana wire model can 
also live on the surface of a 3D class DIII topological superconductor where each chiral Majorana mode is bound between adjacent domains with opposite time reveral breaking phases $\phi= \pm \pi / 2$ (see figure 4.3(b)).[116, 95] The discrete translation order along the $y$-axis perpendicular to the wire direction can be melted by proliferating dislocations (see figure 4.3(c)). With continuous translation symmetry restored, time reversal symmetry becomes local with $T^{2}=-1$ and the coupled Majorana wire model (4.3) recovers the surface Majorana cone (??) in the continuum limit for small $k_{y}$.

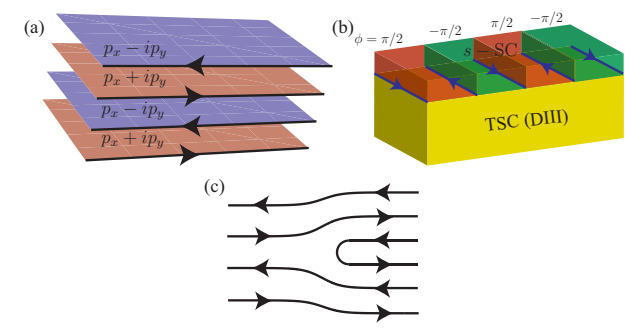

Figure 4.3: Coupled Majorana wire model on the surface of (a) a stack of alternating $p_{x} \pm i p_{y}$ superconductors, and (b) a class DIII topological superconductor (TSC) with alternating TR breaking surface domains. (c) A dislocation.

The non-local time reversal symmetry (4.1) actually provides a weaker topological protection to gapless surface Majorana's than a conventional local one. For instance in section 4.2 , we will show that the $N=2$ coupled Majorana wire model can be gapped by single-body backscattering terms without breaking time reversal, leaving behind a surface with trivial topological order. This reduced robustness stems from the half-translation component in the antiferrormagnetic time reversal. In the BdG description (4.4), the time reversal operator takes the momentum dependent form

$$
T_{\mathbf{k}}=\left(\frac{1+e^{i k_{y}}}{2} \tau_{y}+i \frac{1-e^{i k_{y}}}{2} \tau_{z}\right) \mathcal{K}
$$

for $\mathcal{K}$ the complex conjugation operator. It commutes with the BdG Hamiltonian $T_{\mathbf{k}} H_{\mathrm{BdG}}^{0}(\mathbf{k})=H_{\mathrm{BdG}}^{0}(-\mathbf{k}) T_{\mathbf{k}}$ as well as the particle-hole $(\mathrm{PH}) C T_{\mathbf{k}}=T_{-\mathbf{k}} C$, for $C=\tau_{x} \mathcal{K}$ the $\mathrm{PH}$ operator. In the continuum limit or for small $k_{y}, T \simeq \tau_{y} \mathcal{K}$ agrees with the conventional local time reversal operator and protects a zero en- 
ergy Majorana Kramers' doublet. The BdG Hamiltonian has a chiral symmetry $\Pi_{\mathbf{k}} H_{\mathrm{BdG}}^{0}(\mathbf{k})=-H_{\mathrm{BdG}}^{0}(\mathbf{k}) \Pi_{\mathbf{k}}$, for $\Pi_{\mathbf{k}}=i C T_{\mathbf{k}}$ the chiral operator. It can be used to assign the chirality of a Majorana cone by an integral winding number

$$
n=\frac{1}{2 \pi i} \oint_{\mathcal{C}_{\varepsilon}\left(\mathbf{k}_{0}\right)} \operatorname{Tr}\left[h(\mathbf{k})^{-1} \nabla_{\mathbf{k}} h(\mathbf{k})\right] \cdot d \mathbf{l}
$$

locally around a loop $\mathcal{C}_{\varepsilon}\left(\mathbf{k}_{0}\right) \varepsilon$ away from the zero mode at $\mathbf{k}_{0}$. Here $h(\mathbf{k})$ is the elliptic operator

$$
h(\mathbf{k})=P_{\mathbf{k}}^{+} H_{\mathrm{BdG}}^{0}(\mathbf{k}) P_{\mathbf{k}}^{-}
$$

for $P_{\mathbf{k}}^{ \pm}=\left(P_{\mathbf{k}}^{ \pm}\right)^{2}$ the two local projectors diagonalizing the chiral operator $\Pi_{\mathbf{k}}=$ $e^{-i k_{y} / 2}\left(P_{\mathbf{k}}^{+}-P_{\mathbf{k}}^{-}\right)$. However, as time reversal squares to $T_{\mathbf{k}} T_{-\mathbf{k}}=-e^{i k_{y}}$, which is the eigenvalue of the primitive translation $-\hat{t}_{y}$ at momentum $\mathbf{k}$, so does the nonsymmorphic chiral operator $\Pi_{\mathbf{k}}^{2}=e^{-i k_{y}}$. The two chiral branches $\Pi_{\mathbf{k}}= \pm e^{-i k_{y} / 2}$ switch across the Brillouin zone when $k_{y} \rightarrow k_{y}+2 \pi$. As a result, a global winding number can only be defined modulo 2 .

\subsubsection{The $s o(N)_{1}$ current algebra}

We notice the coupled Majorana wire model (4.3) has a $S O(N)$ symmetry that rotates the $N$-component Majorana fermion $\psi_{y}^{a} \rightarrow O_{b}^{a} \psi_{y}^{b}$. Consequently, there is a chiral so $(N)$ Wess-Zumino-Witten (WZW) theory[136, 139] or affine Kac-Moody algebra at level 1 along each wire. Here we review some relevant features of the $s o(N)_{1}$ algebra, which are well-known and can be found in standard texts on conformal field theory (CFT) such as Ref.[26]. 
The $s o(N)_{1}$ currents have the free field representation

$$
J^{\beta}(z)=\frac{i}{2} \boldsymbol{\psi}(z)^{T} t^{\beta} \boldsymbol{\psi}(z)=\frac{i}{2} \sum_{a b} \psi^{a}(z) t_{a b}^{\beta} \psi^{b}(z)
$$

where the $t^{\beta}$ 's are antisymmetric $N \times N$ matrices that generate the $s o(N)$ Lie algebra (see appendix A), $z=e^{\tau+i x}$ is the complex space-time parameter, and (4.8) is normal ordered. The coupled Majorana wire model carries currents that propagate in alternating directions (see figure 4.1) so that $J_{y}^{\beta}(z)$ are holomorphic for even $y$ and $J_{y}^{\beta}(\bar{z})$ are anti-holomorphic for odd $y$. Focusing on an even wire, from the operator product expansion (OPE)

$$
\psi^{a}(z) \psi^{b}(w)=\frac{\delta^{a b}}{z-w}+\ldots
$$

the $s o(N)_{1}$ currents obey the product expansion

$$
J^{\beta}(z) J^{\gamma}(w)=\frac{\delta^{\beta \gamma}}{(z-w)^{2}}+\sum_{\delta} \frac{i f_{\beta \gamma \delta}}{z-w} J^{\delta}(w)+\ldots
$$

where $f_{\beta \gamma \delta}$ are the structure constants of the $s o(N)$ Lie algebra with $\left[t^{\beta}, t^{\gamma}\right]=$ $\sum_{\delta} f_{\beta \gamma \delta} t^{\delta}$ (see appendix A). The Sugawara energy momentum tensor (along a single wire) is equivalent to the free fermion one[40]

$$
T(z)=\frac{1}{2(N-1)} \mathbf{J}(z) \cdot \mathbf{J}(z)=-\frac{1}{2} \boldsymbol{\psi}(z)^{T} \partial_{z} \boldsymbol{\psi}(z)
$$

for $\mathbf{J}=\left(J^{\beta}\right)$ the current vector and $\boldsymbol{\psi}=\left(\psi^{1}, \ldots, \psi^{N}\right)$ the $N$-component real fermion. The energy momentum tensor defines a chiral Virasoro algebra and characterizes a chiral CFT. It satisfies the OPE

$$
T(z) T(w)=\frac{c_{-} / 2}{(z-w)^{4}}+\frac{2 T(w)}{(z-w)^{2}}+\frac{\partial_{w} T(w)}{z-w}+\ldots
$$


where the chiral central charge $c_{-}=N / 2$, loosely speaking, counts the conformal degrees of freedom on the Majorana wires and is proportional to the energy current[54, 18, 60, 69] and entanglement entropy[3, 50, 16] carried by the wire.

Excitations of the $N$-component Majorana wire transform acording to the $S O(N)$ symmetry. They decompose into primary fields and their corresponding descendants. A primary field $\mathbf{V}_{\lambda}=\left(V^{1}, \ldots, V^{d}\right)$ is a simple excitation sector that irreducibly represents the $s o(N)_{1}$ Kac-Moody algebra.

$$
J^{\beta}(z) V^{r}(w)=-\sum_{s=1}^{d} \frac{\left(t_{\lambda}^{\beta}\right)_{r s}}{z-w} V^{s}(w)+\ldots
$$

where $\lambda$ labels some $d$-dimensional irreducible representation of $s o(N)$ and $t_{\lambda}^{\beta}$ is the $d \times d$ matrix representing the generator $t^{\beta}$ of $s o(N)$. For example it is straightforward to check by using the definition (4.8) and the OPE (4.9) that the Majorana fermion $\boldsymbol{\psi}=\left(\psi^{1}, \ldots, \psi^{N}\right)$ is primary with respect to the fundamental representation, i.e.

$$
J^{\beta}(z) \psi^{a}(w)=-\sum_{b=1}^{N} \frac{t_{a b}^{\beta}}{z-w} \psi^{b}(w)+\ldots
$$

From (4.11), space-time translation of a primary field $\mathbf{V}_{\lambda}$ is governed by

$$
T(z) \mathbf{V}_{\lambda}(w)=\frac{h_{\lambda}}{(z-w)^{2}} \mathbf{V}_{\lambda}(w)+\frac{\partial_{w} \mathbf{V}_{\lambda}(w)}{z-w}+\ldots
$$

where the conformal (scaling) dimension is given by

$$
h_{\lambda}=\frac{\mathcal{Q}_{\lambda}}{2(N-1)}
$$

for $-\sum_{\beta} t_{\lambda}^{\beta} t_{\lambda}^{\beta}=\mathcal{Q}_{\lambda} \mathbb{1}_{d \times d}$ the quadratic Casimir operator. For instance $\mathcal{Q}_{\psi}$, the quadratic Casmir eigenvalue for the fundamental representation, is $N-1$ (see ap- 
pendix A) and therefore the fermion $\boldsymbol{\psi}$ has conformal dimension $h_{\psi}=1 / 2$. This agrees with the OPE (4.9) by dimension analysis.

There are extra primary fields other than the trivial vacuum 1 and the fermion $\psi$. The spinor representations (see appendix A) $\sigma$, for $N$ odd, or $s_{+}$and $s_{-}$, for $N$ even, also correspond to primary fields of $s o(N)_{1}$. Their conformal dimensions can be read off from their quadratic Casmir values (A.7), and are

$$
h_{\sigma}=\frac{N}{16}, \quad h_{s \pm}=\frac{N}{16} .
$$

Unlike the infinite number of irreducible representations of a Lie algebra, the extended

affine $s o(N)_{1}$ algebra only has a truncated set of primary fields $\{1, \sigma, \psi\}$, for $N$ odd, or $\left\{1, s_{+}, s_{-}, \psi\right\}$, for $N$ even.

These $s o(N)_{1}$ primary fields take more explicit operator forms after bosonization and can be found in appendix B and C.

\subsubsection{Bosonizing even Majorana cones}

In the case when $N=2 r$ is even, the $N$ Majorana (real) fermions on each wire can be paired into $r$ Dirac (complex) fermions and bosonized $[140,36]$

$$
c_{y}^{j}=\frac{\psi_{y}^{2 j-1}+i \psi_{y}^{2 j}}{\sqrt{2}} \sim \frac{1}{\sqrt{l_{0}}} \exp \left(i \widetilde{\phi}_{y}^{j}\right)
$$

where $\widetilde{\phi}_{y}^{1}, \ldots, \widetilde{\phi}_{y}^{r}$ are real bosons on the $y^{\text {th }}$ wire, and the vertex operator in $(4.18)$ is normal ordered. The bosons obey the equal-time commutation relation

$$
\begin{aligned}
{\left[\widetilde{\phi}_{y}^{j}(x), \widetilde{\phi}_{y^{\prime}}^{j^{\prime}}\left(x^{\prime}\right)\right]=} & i \pi(-1)^{\max \left\{y, y^{\prime}\right\}}\left[\delta_{y y^{\prime}} \delta^{j j^{\prime}} \operatorname{sgn}\left(x^{\prime}-x\right)\right. \\
& \left.+\delta_{y y^{\prime}} \operatorname{sgn}\left(j-j^{\prime}\right)+\operatorname{sgn}\left(y-y^{\prime}\right)\right]
\end{aligned}
$$


where $\operatorname{sgn}(s)=s /|s|= \pm 1$ for $s \neq 0$ and $\operatorname{sgn}(0)=0$. The first line of (4.19) is equivalent to the commutation relation between conjugate fields

$$
\left[\widetilde{\phi}_{y}^{j}(x), \partial_{x^{\prime}} \widetilde{\phi}_{y^{\prime}}^{j^{\prime}}\left(x^{\prime}\right)\right]=2 \pi i(-1)^{y} \delta_{y y^{\prime}} \delta^{j j^{\prime}} \delta\left(x-x^{\prime}\right)
$$

and is set by the " $p \dot{q} "$ term of the Lagrangian density

$$
\mathcal{L}_{0}=\frac{1}{2 \pi} \sum_{y=-\infty}^{\infty} \sum_{j=1}^{r}(-1)^{y} \partial_{x} \widetilde{\phi}_{y}^{j} \partial_{t} \widetilde{\phi}_{y}^{j}
$$

The second line of (4.19) guarantees the correct anticommutation relations between Dirac fermions along distinct channels. The alternating signs $(-1)^{y}$ in $(4.20)$ and (4.21) specify the propagating directions along each wire, $R$ (or $L$ ) for $y$ even (resp. odd). Eq.(4.19) is symmetric under time reversal (4.1), which sends

$$
\mathcal{T} c_{y}^{j} \mathcal{T}^{-1}=(-1)^{y} c_{y}^{j \dagger}, \quad \mathcal{T} \widetilde{\phi}_{y}^{i} \mathcal{T}^{-1}=\widetilde{\phi}_{y+1}^{i}+\pi y
$$

We notice time reversal, in this convention, flips the fermion parity as it interchanges between the creation and annihilation operators.

The entire coupled Majorana wire Hamiltonian (4.3), when $N=2 r$ is even, can be turned into a model of coupled boson wires. The total Lagrangian density is a combination

$$
\mathcal{L}=\mathcal{L}_{0}-\mathcal{H}=\mathcal{L}_{0}-\left(\mathcal{H}_{\|}+\mathcal{H}_{\perp}\right)
$$

where the Hamiltonian density $\mathcal{H}=\mathcal{H}_{\|}+\mathcal{H}_{\perp}$ consists of the sliding Luttinger liquid[88, 28, 124, 114, 82] (SLL) component along each wire

$$
\mathcal{H}_{\|}=V_{\times} \sum_{y=-\infty}^{\infty} \sum_{j=1}^{r} \partial_{x} \widetilde{\phi}_{y}^{j} \partial_{x} \widetilde{\phi}_{y}^{j}
$$


and the backscattering component between wires

$$
\begin{gathered}
\mathcal{H}_{\perp}=-V_{\mathrm{y}} \sum_{y=-\infty}^{\infty} \sum_{j=1}^{r}(-1)^{y} \cos \left(2 \vartheta_{y+1 / 2}^{j}\right) \\
2 \vartheta_{y+1 / 2}^{j}=\widetilde{\phi}_{y}^{j}-\widetilde{\phi}_{y+1}^{j} .
\end{gathered}
$$

The SLL Hamiltonian (4.24) contains the (normal ordered) kinetic term $i \boldsymbol{\psi}_{y}^{T} \partial_{x} \boldsymbol{\psi}_{y}=$ $i\left(c_{y}^{\dagger} \partial_{x} c_{y}+c_{y} \partial_{x} c_{y}^{\dagger}\right)$ in (4.3) as well as possible forward scattering terms like the densitydensity coupling $\left(c_{y}^{\dagger} c_{y}\right)\left(c_{y}^{\dagger} c_{y}\right)$. The interwire backscattering Hamiltonian (4.25) is identical to the second term $i \boldsymbol{\psi}_{y}^{T} \boldsymbol{\psi}_{y+1}=i\left(c_{y}^{\dagger} c_{y+1}+c_{y} c_{y+1}^{\dagger}\right)$ in (4.3). This can be derived directly by applying the bosonization (4.18) and the Baker-Campbell-Hausdorff formula $e^{i \widetilde{\phi}_{y}} e^{-i \widetilde{\phi}_{y+1}}=e^{i\left(\widetilde{\phi}_{y}-\widetilde{\phi}_{y+1}\right)+\left[\widetilde{\phi}_{y}, \widetilde{\phi}_{y+1}\right] / 2}$. The alternating sign $(-1)^{y}$ in $(4.25)$ is crucial to preserve time reversal symmetry (4.22), which relates $\mathcal{T} 2 \vartheta_{y+1 / 2}^{j} \mathcal{T}^{-1}=2 \vartheta_{y+3 / 2}^{j}-\pi$.

The $r$ sine-Gordon terms in (4.25) between the same pair of adjacent wires mutually commute

$$
\left[2 \vartheta_{y+1 / 2}^{j}(x), 2 \vartheta_{y+1 / 2}^{j^{\prime}}\left(x^{\prime}\right)\right]=0
$$

and share simultaneous eigenvalues. If there was a single pair of counter-propagating wires, these potentials would have pinned $\left\langle 2 \vartheta_{y+1 / 2}^{j}(x)\right\rangle=(2 n+y) \pi$ between the two wires. However, they compete with the sine-Gordon terms between the next pair of wires due to the non-commuting relation

$$
\begin{aligned}
& {\left[2 \vartheta_{y+1 / 2}^{j}(x), 2 \vartheta_{y+3 / 2}^{j^{\prime}}\left(x^{\prime}\right)\right] } \\
= & 2 \pi i(-1)^{y}\left[\theta\left(j-j^{\prime}\right)+\delta^{j j^{\prime}} \theta\left(x^{\prime}-x\right)\right]
\end{aligned}
$$


where the unit step function $\theta(s)=0$ when $s \leq 0$, or 1 when $s>0$. In other words, the vertex operators $e^{i 2 \vartheta_{y+1 / 2}^{j}}$ produces fluctuations to adjacent pairs,

$$
\begin{aligned}
& e^{-i 2 \vartheta_{y+1 / 2}^{j}(x)} 2 \vartheta_{y+3 / 2}^{j}\left(x^{\prime}\right) e^{i 2 \vartheta_{y+1 / 2}^{j}(x)} \\
= & 2 \vartheta_{y+3 / 2}^{j}\left(x^{\prime}\right)+2 \pi(-1)^{y} \theta\left(x^{\prime}-x\right) .
\end{aligned}
$$

The uniform backscattering strength $V_{\mathrm{y}}$, as protected by time reversal (4.1), exactly balances the competing potentials so that the Hamiltonian $\mathcal{H}=\mathcal{H}_{\|}+\mathcal{H}_{\perp}$ remains gapless.

\subsection{Gapping surface Majorana cones}

The previous section describes the gapless surface Majorana fermions of a 3D topological superconductor using a coupled wire model (4.3). It consists of an array of chiral wires, each of which carries $N$ flavors of Majorana fermions co-propagating in alternating directions (see figure 4.1). Together with uniform backscattering interactions between adjacent wires, the model captures $N$ surface Majorana cones with linear energy dispersion about zero energy and momentum (see figure 4.2). In this section we construct explicit fermion interactions that introduce an excitation energy gap to the surface Majorana cones while preserving time reversal symmetry. Generically, this leaves behind a fermionic surface topological order, which will not be discussed until the next section.

We begin with the simplest case when there are $N=2$ chiral Majorana channels along each wire and correspond to two surface Majorana cones. As eluded in section 4.1, due to the non-local nature of time reversal, the coupled wire model can be gapped by single-body backscattering terms without violating the symmetry. Although this cannot be applied to a conventional topological superconductor with local time reversal, this model demonstrates the idea of fractionalization, which can 
be generalized to the many-body interacting case and subsequently lead to surface topological order. The Hamiltonian $\mathcal{H}=\mathcal{H}_{0}+\mathcal{H}_{\mathrm{bc}}$ consists of the original model (4.3) with two fermion flavors $\boldsymbol{\psi}_{y}=\left(\psi_{y}^{1}, \psi_{y}^{2}\right)$ and the inter-flavor backscattering

$$
\mathcal{H}_{\mathrm{bc}}=i u \sum_{y=-\infty}^{\infty} \psi_{y}^{1} \psi_{y+1}^{2}
$$

which is symmetric under the time reversal $(4.1), \mathcal{T}: \psi_{y}^{a} \rightarrow(-1)^{y} \psi_{y+1}^{a}$. The BdG Hamiltonian $H_{\mathrm{BdG}}(\mathbf{k})=H_{\mathrm{BdG}}^{0}(\mathbf{k})+H_{\mathrm{BdG}}^{\mathrm{bc}}(\mathbf{k})$ is the combination of $(4.4)$ and

$$
\begin{aligned}
H_{\mathrm{BdG}}^{\mathrm{bc}}(\mathbf{k})=\frac{u}{2} & {\left[\left(1-\cos k_{y}\right) \sigma_{x} \tau_{z}+\left(1+\cos k_{y}\right) \sigma_{y} \tau_{y}\right.} \\
& \left.-\sin k_{y}\left(\sigma_{y} \tau_{z}+\sigma_{x} \tau_{y}\right)\right]
\end{aligned}
$$

which is symmetric under $T_{\mathbf{k}}$ in (4.5). The energy spectrum depends on the relative strength between the two interwire couplings $i v_{\mathrm{y}}\left(\psi_{y}^{1} \psi_{y+1}^{1}+\psi_{y}^{1} \psi_{y+1}^{1}\right)$ and $i u \psi_{y}^{1} \psi_{y+1}^{2}$ (see figure 4.4). When $u=0$, the two Majorana cone coincide at zero momentum. A finite $u$ separates the two until they have traveled across the Brillouin zone and annihilate each other at $k_{y}=\pi$ when $u>2 v_{\mathrm{y}}$. Once an energy gap has opened up, the BdG Hamitonian has a unit Chern invariant

$$
\mathrm{Ch}=\frac{i}{2 \pi} \int_{-\infty}^{\infty} d k_{x} \int_{-\pi}^{\pi} d k_{y} \operatorname{Tr}\left(\mathcal{F}_{\mathbf{k}}\right)=1
$$

where $\operatorname{Tr}\left(\mathcal{F}_{\mathbf{k}}\right)=\operatorname{Tr}\left(\left\langle\partial_{k_{y}} u_{\mathbf{k}}^{a} \mid \partial_{k_{x}} u_{\mathbf{k}}^{b}\right\rangle-\left\langle\partial_{k_{x}} u_{\mathbf{k}}^{a} \mid \partial_{k_{y}} u_{\mathbf{k}}^{b}\right\rangle\right)$ is the Berry curvature constructed from the two occupied eigenstates $u_{\mathbf{k}}^{1}, u_{\mathbf{k}}^{2}$ below zero energy of $H_{\mathrm{BdG}}(\mathbf{k})$. The coupled Majorana wire model thus behaves like a chiral $p+i p$ topological superconductor[127, 97]. However the single-body Hamiltonian does not possesses a topological order in the sense that it does not support anyonic excitations. For instance the $\psi \rightarrow-\psi \mathbb{Z}_{2}$ symmetry is global and $\pi$-vortices are not quantum excitations of the model but rather introduced as classical extrinsic defects. 


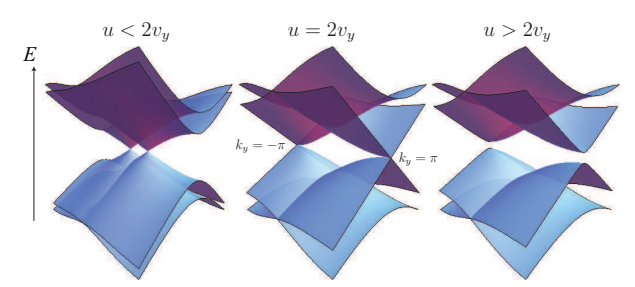

Figure 4.4: Energy spectrum of the $N=2$ coupled Majorana wire model with interflavor mixing.

This example relies on a simple decomposition of the degrees of freedom along each wire, $N=2=1+1$. The two Majorana fermions $\psi_{y}^{1}, \psi_{y}^{2}$ are backscattered independently to adjacent wires in opposite directions. Unlike the intra-flavor couplings $i v_{\mathrm{y}}\left(\psi_{y}^{1} \psi_{y+1}^{1}+\psi_{y}^{1} \psi_{y+1}^{1}\right)$, inter-flavor terms $i u \psi_{y}^{1} \psi_{y+1}^{2}$ freeze independent degrees of freedom and they are not competing with each other. It is useful to notice that the decomposition breaks the $S O(2)_{1}$ symmetry described in section 4.1.1, and as a result the $s o(2 r)_{1}$ CFT along each wire splits into a pair of chiral Ising CFT's.

We can now generalize this idea to all $N$, but with many-body interwire interactions. From now on, unless specified otherwise, we turn off all single-body scattering terms. For instance, the vertical velocity now vanishes, $v_{\mathrm{y}}=0$, in the kinetic part $\mathcal{H}_{0}$ of the coupled wire model (4.3). First we seek a decomposition of the $s o(N)_{1}$ degrees of freedom along each wire (see section 4.1.1) into a pair of identical but independent sectors (also see figure 4.1)

$$
s o(N)_{1} \supseteq \mathcal{G}_{N}^{+} \times \mathcal{G}_{N}^{-}
$$

where $\mathcal{G}_{N}^{ \pm}$are the Kac-Moody subalgebras

$$
\mathcal{G}_{N}^{ \pm}= \begin{cases}s o(N / 2)_{1} & \text { for } N \text { even } \\ s o(3)_{3} \times s o\left(\frac{N-9}{2}\right)_{1} & \text { for } N \text { odd }\end{cases}
$$


to be discussed below. This fractionalization has to be complete in the sense that the Sugawara energy-momentum tensor exactly splits into

$$
T_{s o(N)_{1}}=T_{\mathcal{G}_{N}^{+}}+T_{\mathcal{G}_{N}^{-}}
$$

In particular the central charge divides

$$
c_{-}\left(s o(N)_{1}\right)=2 c_{-}\left(\mathcal{G}_{N}\right)=c_{-}\left(\mathcal{G}_{N}^{+}\right)+c_{-}\left(\mathcal{G}_{N}^{-}\right)
$$

and there are no degrees of freedom left behind. Using the subalgebra current operators $\mathbf{J}_{\mathcal{G}_{N}^{ \pm}}$, which are quadratic in $\psi$ 's, we construct the four-fermion backscattering interaction

$$
\begin{aligned}
\mathcal{H}_{\text {int }} & =u \sum_{y=-\infty}^{\infty} \mathbf{J}_{\mathcal{G}_{N}^{-}}^{y} \cdot \mathbf{J}_{\mathcal{G}_{N}^{+}}^{y+1} \\
& =u \sum_{y^{\prime}=-\infty}^{\infty} \mathbf{J}_{\mathcal{G}_{N}^{L,-}}^{2 y^{\prime}-1} \cdot \mathbf{J}_{\mathcal{G}_{N}^{R,+}}^{2 y^{\prime}}+\mathbf{J}_{\mathcal{G}_{N}^{R,-}}^{2 y^{\prime}} \cdot \mathbf{J}_{\mathcal{G}_{N}^{L,+}}^{2 y^{\prime}+1}
\end{aligned}
$$

for $u$ positive, and $R, L$ labels the propagating directions of the currents. This is pictorially presented in figure 4.1 and 4.5 .

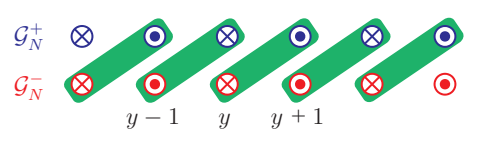

Figure 4.5: Interwire gapping terms (4.37) (green rectangular boxes) between chiral fractional $\mathcal{G}_{N}^{R, \pm}, \mathcal{G}_{N}^{L, \pm}$ sectors (resp. $\otimes, \odot$ ) in opposite direction.

In this section, we design the fractionalization (4.33) of $s o(N)_{1}$ for all $N$ and show that the backscattering interactions (4.37) open an excitation energy gap without breaking time reversal. In CFT context, (4.33) is also known as a conformal 
embedding $[26,11,108,10]$. When $N=2 r$ is even, there is an obvious decomposition

$$
s o(2 r)_{1} \supseteq s o(r)_{1}^{+} \times s o(r)_{1}^{-}
$$

where the "+" sector contains $\psi^{1}, \ldots, \psi^{r}$ while the "-" one contains the rest $\psi^{r+1}, \ldots, \psi^{2 r}$. In section 4.2.1, we review how the $\mathbf{J}_{s o(r)_{1}^{R}} \cdot \mathbf{J}_{s o(r))_{1}^{L}}$ interactions contribute an energy gap. This is a direct application of the well-studied $O(N)$ Gross-Neveu problem $[43,145,138,113]$ in 1D. In the discrete limit, this is related to the Haldane $O(3)$ antiferrormagnetic spin chain[44, 45], the Affleck - Kennedy Lieb - Tasaki (AKLT) spin chains[1, 2] and the $S O(n)$ Heisenberg chain[120, 119, 4]. When $N$ is odd, the splitting (4.33) is less trivial. We will make use of the level-rank duality[26, 84, 76]

$$
s o\left(n^{2}\right)_{1} \supseteq s o(n)_{n} \times s o(n)_{n}
$$

which comes from the fact that the tensor product $S O(n) \otimes S O(n)$ is a Lie subgroup in $S O\left(n^{2}\right)$. In particular, we will demonstrate the simplest case in section 4.2.2 when $n=3$. The division of $s o(9)_{1}$ can subsequently be generalized to $s o(N)_{1}$ for all odd $N$ effectively by writing $N=9+2 r$. This sets $\mathcal{G}_{N}^{ \pm}=s o(3)_{3} \times s o(r)_{1}$ in (4.33) and the corresponding interwire backscattering interactions (4.37).

\subsubsection{Gapping even Majorana cones}

We begin with the coupled Majorana wire model (4.3) (or figure 4.1) with $N=2 r$ chiral fermion channels per wire and corresponds to the same number of gapless Majorana cones. Similar to the previously shown $N=2$ case, the gapless modes can be removed using simple single-body backscattering terms. We however are interested in finding gapping interactions that would support surface topological order as well. In section 4.1.1 and appendices B, C, we described the $s o(N)_{1}$ WZW theory, which 
along the $y^{\text {th }}$ wire is generated by chiral current operators (4.8)

$$
J_{y}^{(a, b)}=(-1)^{y} i \psi_{y}^{a} \psi_{y}^{b}
$$

We take the alternating sign convention $(-1)^{y}$ so that under time reversal, $\mathcal{T} J_{y}^{(a, b)} \mathcal{T}^{-1}=J_{y+1}^{(a, b)}$. We consider two subsets of generators, $s o(r)_{1}^{+}$containing $J^{(a, b)}$ for $1 \leq a<b \leq r$, and $s o(r)_{1}^{-}$containing $J^{(a, b)}$ for $r+1 \leq a<b \leq 2 r$. As they act on independent fermion sectors, the two sets of operators commute or equivalently their operator product expansions (OPE) are trivial up to non-singular terms. Moreover the Sugawara energy-momentum tensor (4.11) for $s o(N)_{1}$ completely splits into a sum between

$$
T_{s o(r)_{1}^{+}}=-\frac{1}{2} \sum_{a=1}^{r} \psi^{a} \partial \psi^{a}, \quad T_{s o(r)_{1}^{-}}=-\frac{1}{2} \sum_{a=r+1}^{2 r} \psi^{a} \partial \psi^{a} .
$$

This ensures all degrees of freedom in $s o(2 r)_{1}$ are generated by tensor products between those in the $s o(r)_{1}^{ \pm}$sectors. Precisely this means any $s o(2 r)_{1}$ primary field is a fusion channel of the OPE of certain primary field pair in $s o(r)_{1}^{+}$and $s o(r)_{1}^{-}$. Thus as long as the gapping terms independently freeze both sectors, they remove all gapless degrees of freedom.

The backscattering interactions (4.37) couples the $s o(r)_{1}^{-}$sector on the $y^{\text {th }}$ wire with the $s o(r)_{1}^{+}$sector on the $(y+1)^{\text {th }}$ one. They can explicitly written as

$$
\mathcal{H}_{\text {int }}=u \sum_{y=-\infty}^{\infty} \sum_{1 \leq a<b \leq r} \psi_{y}^{r+a} \psi_{y}^{r+b} \psi_{y+1}^{a} \psi_{y+1}^{b}
$$

Firstly, the interactions are time reversal symmetric as (4.42) is unchanged by $\psi_{y}^{a} \rightarrow$ $(-1)^{y} \psi_{y+1}^{a}$. Secondly, it breaks the $O(2 r)$ symmetry to $O(r)^{+} \times O(r)^{-}$. The symmetry breaking can be facilitated by forward scattering within wires that renormalizes the velocities differently between the $s o(r)_{1}^{ \pm}$sectors. Eq.(4.42) is also a combination 
allowed by the chiral $O(r)$ symmetry

$$
\psi_{y}^{a} \rightarrow\left(\mathcal{O}^{(-1)^{y}}\right)_{b}^{a} \psi_{y}^{b}, \quad \psi_{y}^{r+a} \rightarrow\left(\mathcal{O}^{(-1)^{y+1}}\right)_{b}^{a} \psi_{y}^{r+b}
$$

where $\mathcal{O}$ is a $r \times r$ orthogonal transformation matrix. The chiral symmetry only allows cross couplings $\mathbf{J}_{s o(r)_{1}^{ \pm}}^{y} \cdot \mathbf{J}_{s o(r)_{1}^{\mp}}^{y+1}$ between adjacent wires. Instead of (4.42), another possibility would be its mirror image with summands $\psi_{y}^{a} \psi_{y}^{b} \psi_{y+1}^{r+a} \psi_{y+1}^{r+b}$. This competes with the original, but as long as mirror symmetry is broken and their strength is asymmetric, an energy gap will open. In the following we will ignore the mirror image by assuming it is weaker.

Next we notice that the four-fermion interaction (4.42) is marginally relevant when velocity $v_{\mathrm{x}}$ is uniform. The dimensionless coupling strength $u$ follows the renormalization group ( $\mathrm{RG})$ flow equation

$$
\frac{d u}{d \lambda}=+4 \pi(r-2) u^{2}
$$

when length scale renormalizes by $l \rightarrow e^{\lambda} l$. This can be verified by applying the RG formula among marginal operators[19]

$$
\frac{d g_{l}}{d \lambda}=-2 \pi \sum_{m n} C_{l}^{m n} g_{m} g_{n}
$$

where $C_{l}^{m n}$ is the fusion coefficient of the OPE $\mathcal{O}_{m} \mathcal{O}_{n}=C_{l}^{m n} \mathcal{O}_{l}+\ldots$ between operators in the perturbative action $\delta S=\int d \tau d x \sum_{m} g_{m} \mathcal{O}_{m}$. In the current case, the fusion coefficient $\mathcal{O O}=-2(r-2) \mathcal{O}+\ldots$ can be evaluated simply by applying the Wick's theorem of fermions, for $\mathcal{O}=-\sum_{y, a, b} \psi_{y}^{r+a} \psi_{y}^{r+b} \psi_{y+1}^{a} \psi_{y+1}^{b}$. The plus sign in (4.44) shows the interacting strength grows at weak coupling. To show that the backscattering (4.42) indeed opens up a gap, we first focus on a single coupled pair of counter-propagating $s o(r)_{1}$ channels (see figure 4.5). 


\subsubsection{The $O(r)$ Gross-Neveu model}

Here we concentrate on a particular set of backscattering terms in (4.42) at say an even $y$. We relabel $\psi_{y}^{r+a}=\psi_{R}^{a}$ and $\psi_{y+1}^{a}=\psi_{L}^{a}$, for $a=1, \ldots, r$. The interaction between the $y^{\text {th }}$ and $(y+1)^{\text {th }}$ wire is identical to that of the $O(r)$ Gross-Neveu (GN) $\operatorname{model}[43,145,138,113]$

$$
\mathcal{H}_{\mathrm{GN}}=-\frac{u}{2}\left(\boldsymbol{\psi}_{R} \cdot \boldsymbol{\psi}_{L}\right)^{2}
$$

where the minus sign is from the fermion exchange statistics $\psi_{R}^{a} \psi_{R}^{b} \psi_{L}^{a} \psi_{L}^{b}=$ $-\psi_{R}^{a} \psi_{L}^{a} \psi_{R}^{b} \psi_{L}^{b}$. This GN model is known to have an excitation energy gap for $r>2$.

For even $r=2 n>2$, the Majorana fermions can be paired into Dirac ones and subsequently bosonized (see section 4.1.2), $c_{R / L}^{j}=\left(\psi_{R / L}^{2 j-1}+i \psi_{R / L}^{2 j}\right) / \sqrt{2} \sim e^{i \widetilde{\phi}_{R / L}^{j}}$, for $j=1, \ldots, n$. Using

$$
\boldsymbol{\psi}_{R} \cdot \boldsymbol{\psi}_{L}=\sum_{j=1}^{n} c_{R}^{j}\left(c_{L}^{j}\right)^{\dagger}+\left(c_{R}^{j}\right)^{\dagger} c_{L}^{j} \sim \sum_{j=1}^{n} \cos \left(2 \Theta^{j}\right)
$$

for $2 \Theta^{j}=\widetilde{\phi}_{R}^{j}-\widetilde{\phi}_{L}^{j}$ (also see (4.26)) are mutually commuting variables, the GN interation (4.46) takes the bosonized form

$$
\begin{aligned}
\mathcal{H}_{G N} & \sim u \sum_{j=1}^{n} \partial_{x} \widetilde{\phi}_{R}^{j} \partial_{x} \widetilde{\phi}_{L}^{j}-u \sum_{j_{1} \neq j_{2}} \sum_{ \pm} \cos \left(2 \Theta^{j_{1}} \pm 2 \Theta^{j_{2}}\right) \\
& =u \sum_{j=1}^{n} \partial_{x} \widetilde{\phi}_{R}^{j} \partial_{x} \widetilde{\phi}_{L}^{j}-u \sum_{\boldsymbol{\alpha} \in \Delta} \cos (\boldsymbol{\alpha} \cdot 2 \boldsymbol{\Theta})
\end{aligned}
$$

where $2 \boldsymbol{\Theta}=\left(2 \Theta^{1}, \ldots, 2 \Theta^{n}\right)$ and $\boldsymbol{\alpha}$ are roots of $s o(2 n)$ (see (A.8)). The first term renormalizes the velocity $V_{x}$ in (4.24) as well as the Luttinger parameter. We assume $V_{\mathrm{x}}>>u$ so that the first term can be dropped. The remaining sine-Gordon terms are responsible for gapping out all low energy degrees of freedom. Firstly the angle 
parameters mutually commute and share simultaneous eigenvalues. The ground state minimizes the energy by uniformly pinning the ground state expectation value (GEV)

$$
\left\langle 2 \Theta^{j}(x)\right\rangle=\pi m_{\psi}^{j}, \quad m_{\psi}^{j} \in \mathbb{Z}
$$

We notice in passing that the following subset of sine-Gordon terms

$$
\begin{aligned}
-u \sum_{I=1}^{n} \cos \left(\boldsymbol{\alpha}_{I} \cdot 2 \boldsymbol{\Theta}\right) & =-u \sum_{I=1}^{n} \cos \left[\sum_{J=1}^{n} K_{I J}\left(\phi_{R}^{J}-\phi_{L}^{J}\right)\right] \\
& =-u \sum_{I=1}^{n} \cos \left(\mathbf{n}_{I}^{T} \mathbb{K} \boldsymbol{\Phi}\right)
\end{aligned}
$$

using the simple roots $\boldsymbol{\alpha}_{I}$ in (A.9), is already enough to remove all low energy degrees of freedom. Here $K_{I J}$ is the Cartan matrix (A.12) of $s o(2 n)$ that appears in the Lagrangian density

$$
\mathcal{L}_{0}=\frac{1}{2 \pi} \partial_{x} \boldsymbol{\Phi}^{T} \mathbb{K} \partial_{t} \boldsymbol{\Phi}
$$

for $\mathbb{K}=K \oplus(-K)$ and $\boldsymbol{\Phi}=\left(\boldsymbol{\phi}_{R}, \boldsymbol{\phi}_{L}\right)$, and $\phi$ is related to $\widetilde{\phi}$ by the basis transformation (B.13). For instance, the $n$ vector coefficients $\mathbf{n}_{J}=\left(\mathbf{e}_{J}, \mathbf{e}_{J}\right)$ in (4.50) form a null basis

$$
\mathbf{n}_{I}^{T} \mathbb{K} \mathbf{n}_{J}=0
$$

and guarantee an energy gap according to Ref.[46]. The remaining GN terms in (4.48) are compatible with (4.50) as they share the same minima.

There are constraints on the GEV $m_{\psi}^{j}$ in (4.49). In order to minimize $-\cos (\boldsymbol{\alpha} \cdot 2 \boldsymbol{\Theta})$ in (4.48), $\langle\boldsymbol{\alpha} \cdot 2 \boldsymbol{\Theta}\rangle$ must be an integer multiple of $2 \pi$. This restricts uniform parity 
among $m_{\psi}^{j}$ so that the sign in the fermion backscattering amplitude

$$
\begin{aligned}
\left\langle\psi_{R}^{a}(x) \psi_{L}^{a}(x)\right\rangle & =\left\langle c_{R}^{j}(x) c_{L}^{j}(x)^{\dagger}\right\rangle \\
& \sim\left\langle e^{i 2 \Theta^{j}(x)}\right\rangle=(-1)^{m_{\psi}} .
\end{aligned}
$$

does not depend on fermion flavor $j$. This is not the only non-zero GEV as $\psi$ is not the only primary field in $s o(2 n)_{1}$. The backscattering of spinor fields $V_{s_{ \pm}}=e^{i \varepsilon \cdot \widetilde{\phi} / 2}$ (B.24) corresponds to the two GEV's

$$
\left\langle V_{s_{ \pm}}^{R}(x) V_{s_{ \pm}}^{L}(x)^{\dagger}\right\rangle=\left\langle e^{i \varepsilon \cdot \Theta(x)}\right\rangle=e^{i \pi m_{s_{ \pm}} / 2}
$$

where $\varepsilon=\left(\varepsilon_{1}, \ldots, \varepsilon_{n}\right)$ for $\varepsilon_{j}= \pm 1$, and the overall sign $\prod_{j} \varepsilon_{j}$ is positive for the even spinor field $s_{+}$, or negative for $s_{-}$. Here the GEV (4.54) does not depend on the choice of $\varepsilon$. This is because given $\varepsilon$ and $\varepsilon^{\prime}$ with the same overall parity $\prod \varepsilon_{j}=\prod \varepsilon_{j}^{\prime}$, $\boldsymbol{\varepsilon} \cdot \boldsymbol{\Theta}$ and $\boldsymbol{\varepsilon}^{\prime} \cdot \boldsymbol{\Theta}$ differ by some combination of $\boldsymbol{\alpha} \cdot 2 \boldsymbol{\Theta}$, which takes expectation value in $2 \pi \mathbb{Z}$.

There are extra constraints between $m_{\psi}$ and $m_{s_{ \pm}}$from the fusion rules of the primary fields of $s o(2 n)_{1}\left(\right.$ see $(\mathrm{B} .25)$ and (B.26)). Firstly, $s_{ \pm} \times \psi=s_{\mp}$ requires

$$
m_{s_{+}} \equiv m_{s_{-}}+2 m_{\psi} \quad \bmod 4 \mathbb{Z}
$$

Take the highest weights $\varepsilon_{+}^{0}=(1, \ldots, 1)$ and $\varepsilon_{-}^{0}=(1, \ldots,-1)$ for instance. $\varepsilon_{+}^{0} \cdot \boldsymbol{\Theta}=$ $\varepsilon_{-}^{0} \cdot \Theta+2 \Theta^{n}$ imples $m_{s_{+}}\left(\varepsilon_{+}^{0}\right)=m_{s_{-}}\left(\varepsilon_{+}^{0}\right)+2 m_{\psi}^{n}$. Lastly the fusion rules

$$
s_{ \pm} \times s_{ \pm} \begin{cases}1, & \text { for } n \text { even } \\ \psi, & \text { for } n \text { odd }\end{cases}
$$


requires the GEV's to obey

$$
\begin{cases}(-1)^{m_{s_{ \pm}}}=1 & \text { for } n \text { even } \\ (-1)^{m_{s_{ \pm}}}=(-1)^{m_{\psi}} & \text { for } n \text { odd }\end{cases}
$$

for similar reasons.

The GN model therefore has four ground states when $r=2 n>2$. They are specified by the quantum numbers (i) $m_{s_{+}}=0,1,2,3$ modulo 4 when $n$ is odd, or (ii) $m_{s_{+}}=0,2$ and $m_{s_{-}}=0,2$ modulo 4 when $n$ is even. The rest are fixed by (4.55) and (4.57). Quasiparticle excitations are trapped between domain walls or kinks separating distinct ground states[138, 113, 31]. For example, the vertex operator $V_{s_{+}}^{R}\left(x_{0}\right)=e^{i \varepsilon_{+}^{0} \cdot \widetilde{\phi}_{R}\left(x_{0}\right) / 2}$ of an even spinor field creates a jump in the GEV (4.53)

$$
\left\langle V_{s_{+}}^{R}\left(x_{0}\right)^{\dagger} e^{i 2 \Theta^{j}(x)} V_{s_{+}}^{R}\left(x_{0}\right)\right\rangle=(-1)^{m_{\psi}^{\prime}+\theta\left(x_{0}-x\right)}
$$

because of the Baker-Hausdorff-Campbell formula and the commutation relation from (4.19)

$$
\left[2 \Theta^{j}(x), \boldsymbol{\varepsilon}_{+}^{0} \cdot \widetilde{\boldsymbol{\phi}}_{R}\left(x_{0}\right) / 2\right]=i \pi\left(\theta\left(x_{0}-x\right)-n+j-1\right)
$$

for $\theta$ the unit step function $\theta(s)=0$ when $s \leq 0$, or 1 when $s>0$, and $m_{\psi}^{\prime}=$ $m_{\psi}+n-j+1$. In general, the primary fields $V_{s_{ \pm}}^{R}=e^{i \varepsilon \cdot \widetilde{\boldsymbol{\phi}}_{R}}$ and $c_{R}^{j}=e^{i \widetilde{\phi}_{R}^{j}}$ corresponds to the domain walls of $m_{s_{ \pm}}$:

$$
\begin{aligned}
\left\langle V_{s_{ \pm}}^{R}\left(x_{0}\right)^{\dagger} e^{i \varepsilon_{ \pm}^{0} \cdot \Theta(x)} V_{s_{ \pm}}^{R}\left(x_{0}\right)\right\rangle & =e^{\frac{i \pi}{2}\left(m_{s_{ \pm}}^{\prime}+n \theta\left(x_{0}-x\right)\right)} \\
\left\langle V_{s_{\mp}}^{R}\left(x_{0}\right)^{\dagger} e^{i \varepsilon_{ \pm}^{0} \cdot \Theta(x)} V_{s_{\mp}}^{R}\left(x_{0}\right)\right\rangle & =e^{\frac{i \pi}{2}\left(m_{s_{ \pm}}^{\prime}+(n-2) \theta\left(x_{0}-x\right)\right)} \\
\left\langle c_{R}^{j}\left(x_{0}\right)^{\dagger} e^{i \varepsilon_{ \pm}^{0} \cdot \Theta(x)} c_{R}^{j}\left(x_{0}\right)\right\rangle & =e^{\frac{i \pi}{2}\left(m_{s_{ \pm}}^{\prime}+2 \theta\left(x_{0}-x\right)\right)} .
\end{aligned}
$$


Now we move on to the odd $r=2 n+1>1$ case. First we pair the first $2 n$ Majorana fermions into $n$ Dirac ones and bosonize them similar to the previous even $r$ case. This leaves a single unpaired Majorana fermion $\psi_{R / L}^{r}$. Dropping terms that only renormalizes velocities, the GN model (4.46) takes the partially bosonized form

$$
\begin{aligned}
& \mathcal{H}_{\mathrm{GN}} \sim-u \sum_{\boldsymbol{\alpha} \in \Delta_{s o(2 n)}} \cos (\boldsymbol{\alpha} \cdot 2 \boldsymbol{\Theta}) \\
&-u\left[\sum_{j=1}^{n} \cos \left(2 \Theta^{j}\right)\right] i \psi_{R}^{r} \psi_{L}^{r}
\end{aligned}
$$

where the first line is identical to the even $r$ case (4.61) and is responsible for gapping out first $2 n$ Majorana channels. Projecting onto the lowest energy states and taking the GEV $\left\langle\cos \left(2 \Theta^{j}\right)\right\rangle=(-1)^{m_{\psi}}$, the interacting Hamiltonian becomes

$$
\mathcal{H}_{\mathrm{GN}} \sim-2 n(n-1) u-n u(-1)^{m_{\psi}} i \psi_{R}^{r} \psi_{L}^{r}
$$

which is identical to the continuum limit of the quantum Ising model with transverse field after a Jordan-Wigner transformation. The remaining Majorana channel $\psi_{R / L}^{r}$ is gapped by the single-body backscattering term. The sign of the mass gap $n u(-1)^{m_{\psi}}$ determines the phase of the Ising model. We take the convention so that a negative (or positive) mass with $m_{\psi} \equiv 1$ (resp. $m_{\psi} \equiv 0$ ) corresponds to the order (resp. disorder) phase.

Like the previous case, the fermion backscattering amplitude (4.53) is not the only ground state expectation value. From (C.5) appendix C, the Ising twist field of $s o(2 n+1)_{1}$ can be written as the product $V_{\sigma}=e^{i \varepsilon \cdot \widetilde{\phi} / 2} \sigma^{r}$, where $\varepsilon=\left(\varepsilon_{1}, \ldots, \varepsilon_{n}\right)$ for $\varepsilon_{j}= \pm 1$, and $\sigma_{R / L}^{r}=\sigma_{R / L}^{2 n+1}$ is the twist field along the last Majorana channel. There 
are three possible GEV for the backscattering

$$
\begin{aligned}
\left\langle V_{\sigma}^{R}(x) V_{\sigma}^{L}(x)^{\dagger}\right\rangle & =\left\langle e^{i \varepsilon \cdot \Theta(x)} \sigma_{R}^{r}(x) \sigma_{L}^{r}(x)\right\rangle \\
& \sim\left\{\begin{array}{cl}
0 & \text { for the disorder phase } \\
\pm 1 & \text { for the order phase }
\end{array}\right.
\end{aligned}
$$

Here we choose the convention so that $\sigma_{R} \sigma_{L}$ takes the role of the spin operator $\boldsymbol{\sigma}$ in the Ising model and its non-trivial GEV's in the order phase specify two ground states $|\uparrow\rangle$ and $|\downarrow\rangle$.

Again, quasiparticle excitations are trapped between domain walls separating distinct ground states[138, 113, 31]. For example a twist field $V_{\sigma}^{R}\left(\right.$ or $\left.V_{\sigma}^{L}\right)$ sits between the order to disorder phase boundary where the quantum number $m_{\psi}$ flips from 1 to 0 , or equivalently the fermion mass gap in (4.62) changes sign. This is because the twist field $V_{\sigma}^{R}\left(x_{0}\right)$ introduces a flip in boundary condition $\psi_{R}\left(x_{0}+\right)=-\psi_{R}\left(x_{0}-\right)$ and corresponds to a change of sign in front of the fermion backscattering $i \psi_{R} \psi_{L}$. Alternatively, this can also be understood by identifying $V_{\sigma}$ as a Jackiw-Rebbi soliton[52] or a zero energy Majorana bound state between a trivial and topological superconductor[61] in $1 D$.

Next a $\uparrow-\downarrow$ domain wall of opposite signs of the GEV (4.63) in the order phase traps an excitation in the fermion sector $\psi$. This can be seen by equating the order Ising phase to a 1D topological superconductor[61], where the two Ising ground states corresponds to the even and odd fermion parity states among the pair of boundary Majorana zero modes. Adding (or subtracting) a fermion therefore flips the parity as well as the GEV in (4.63). We notice this domain wall interpretation of excitations is consistent with the non-Abelian fusion rule

$$
\sigma \times \sigma=1+\psi
$$


The trivial fusion channel corresponds to the annihilation of a domain wall pair such as

$$
|\underbrace{\ldots \uparrow \uparrow}_{\text {order }} \underbrace{\leftarrow \leftarrow}_{\text {disorder }} \underbrace{\uparrow \uparrow \ldots\rangle}_{\text {order }} \stackrel{\text { fusion }}{\longrightarrow}| \ldots \uparrow \uparrow \ldots\rangle
$$

while the fermion fusion channel corresponds to joining the pair of "order - disorder" domain walls into a kink

$$
|\underbrace{\ldots \uparrow \uparrow}_{\text {order }} \underbrace{\leftarrow \leftarrow}_{\text {disorder }} \underbrace{\downarrow \downarrow \ldots}_{\text {order }}\rangle \stackrel{\text { fusion }}{\longrightarrow}|\ldots \uparrow \uparrow \downarrow \downarrow . .\rangle .
$$

\subsubsection{The special case: $s o(4)_{1}=s u(2)_{1} \times s u(2)_{1}$}

The case when $r=2$ requires special attention. The $O(2)$ GN model (4.46) is a gapless Luttinger liquid because its bosonized form (4.48) contains no sine-Gordon terms and the rest only renormalizes velocities and the Luttinger parameter. As a result the fractionalization (or conformal embedding) $s o(4)_{1} \supseteq s o(2)_{1} \times s o(2)_{1}$ of wires with $N=4$ Majorana channels does not lead to a gapped theory. Instead we turn to an alternative fractionalization $s o(4)_{1}=s u(2)_{1}^{+} \times s u(2)_{1}^{-}$that only applies for $N=4$.

The four Majorana $\psi_{y}^{a}$ along each wire can be paired into Dirac channels $c_{y}^{1}=$ $\left(\psi_{y}^{1}+i \psi_{y}^{2}\right) / \sqrt{2}=e^{i \widetilde{\phi}_{y}^{1}}$ and $c_{y}^{2}=\left(\psi_{y}^{3}+i \psi_{y}^{4}\right) / \sqrt{2}=e^{i \widetilde{\phi}_{y}^{2}}$. It will be more convenient if we express the bosons in the new basis using the simple roots of $s o(4): \widetilde{\phi}^{1}=\phi^{1}-\phi^{2}$ and $\widetilde{\phi}^{2}=\phi^{1}+\phi^{2}$. Unlike when $r>2$, these bosons decouple in the Lagrangian density

$$
\mathcal{L}_{0}=\frac{1}{2 \pi} \sum_{y=-\infty}^{\infty}(-1)^{y} \sum_{J=1}^{2} 2 \partial_{x} \phi_{y}^{J} \partial_{t} \phi_{y}^{J} .
$$


This is equivalent to the fact that the Cartan matrix $K_{s o(4)}=\operatorname{diag}(2,2)$ is diagonal so that the Lie algebra splits into the product $s u(2)^{+} \times s u(2)^{-}$of isoclinic rotations, each with Cartan matrix $K_{s u(2)}=2$.

The $s u(2)_{1}$ current generators are given by $S_{\mathbf{z}}^{I}(z)=i \sqrt{2} \partial \phi^{I}(z)$ and $S_{ \pm}^{I}(z)=$ $\left(S_{\mathrm{x}}^{I} \pm i S_{\mathrm{y}}^{I}\right) / \sqrt{2}=e^{i 2 \phi^{I}(z)}$, and they satisfy the OPE

$$
S_{\mathrm{i}}^{I}(z) S_{\mathrm{j}}^{I}(w)=\frac{\delta_{\mathrm{ij}}}{(z-w)^{2}}+\frac{i \sqrt{2} \varepsilon_{\mathrm{ijk}}}{z-w} S_{\mathrm{k}}^{I}(w)+\ldots
$$

for $I=1,2=+,-$. The $s u(2)_{1}^{+}$sector is completely decoupled from the $s u(2)_{1}^{-}$one as the OPE $S_{\mathrm{i}}^{1}(z) S_{\mathrm{j}}^{2}(w)$ is non-singular. They completely decomposes all low energy degrees of freedom as the energy momentum tensor splits into

$$
\begin{aligned}
T_{s o(4)_{1}} & =-\frac{1}{2} \sum_{j=1}^{2} \partial \widetilde{\phi}^{j}(z) \partial \widetilde{\phi}^{j}(z) \\
& =-\sum_{J=1}^{2} \partial \phi^{J}(z) \partial \phi^{J}(z)=T_{s u(2)_{1}^{+}}+T_{s u(2)_{1}^{-}}
\end{aligned}
$$

The gapping Hamiltonian is

$$
\begin{aligned}
\mathcal{H}_{\text {int }} & =u \sum_{y=-\infty}^{\infty} \mathbf{S}_{y}^{2} \cdot \mathbf{S}_{y+1}^{1} \\
& =2 u \sum_{y=-\infty}^{\infty} \partial_{x} \phi_{y}^{2} \partial_{x} \phi_{y+1}^{1}-2 \cos \left(4 \Theta_{y+1 / 2}\right) \\
4 \Theta_{y+1 / 2} & =2 \phi_{y+1}^{1}-2 \phi_{y}^{2} \\
& =\widetilde{\phi}_{y+1}^{1}+\widetilde{\phi}_{y+1}^{2}+\widetilde{\phi}_{y}^{1}-\widetilde{\phi}_{y}^{2} .
\end{aligned}
$$

The first kinetic term of the interacting Hamiltonian only renormalizes velocities and the Luttinger parameter. The second sine-Gordon term involves four-fermion interactions and is responsible for the energy gap as it back-scatters the $s u(2)_{1}^{-}$sector on the $y^{\text {th }}$ wire to the $s u(2)_{1}^{+}$sector on the $(y+1)^{\text {th }}$ one. It pins the ground state 
expectation value (GEV)

$$
\left\langle e^{i 2 \Theta_{y+1 / 2}(x)}\right\rangle=(-1)^{m_{s}}
$$

which characterizes the two distinct ground states. Like the previous cases, quasiparticle excitations are kinks in the GEV. The fundamental excitation can be created by the vertex operator $V_{s}=e^{i \phi_{y+1}^{1}}$, which is the semionic primary field in the $s u(2)_{1}^{+}$ sector along the $(y+1)^{\text {th }}$ wire.

\subsubsection{Gapping odd Majorana cones}

We now move on to the case when there are $N=2 r+1 \geq 3$ chiral Majorana channels on each wire in the coupled Majorana wire model (4.3) (of figure 4.1). It corresponds to an odd $N$ number of Majorana cones on the surface of a 3D topological superconductor. The chiral degrees of freedom along each wire are described by a $s o(N)_{1}$ WZW theory, which is going to be fractionalized into the pair $\mathcal{G}_{N}^{+} \times \mathcal{G}_{N}^{-}$ according to $(4.34)$. The $\mathcal{G}_{N}^{-}$sector along the $y^{\text {th }}$ wire will then be back-scattered onto the $\mathcal{G}_{N}^{+}$sector along the $(y+1)^{\text {th }}$ one by the current-current interaction $(4.37)$, which will introduce an energy gap.

Unlike the even $N$ case where $s o(N)_{1}$ can simply be split into a pair of $s o(N / 2)_{1}$ 's, here the decomposition is less trivial but leads to more exotic surface topological order. We begin with the particular case where 9 Majorana channels can be fractionalized into

$$
s o(9)_{1} \supseteq s o(3)_{3} \times s o(3)_{3}
$$

essentially by noticing that the tensor product $S O(3) \otimes S O(3)$ sits inside $S O(9)$. The two $s o(3)_{3}$ WZW sectors carry decoupled current generators. They can then be back- 
scattered using the current-current interaction (4.37) onto adjacent wires in opposite directions (also see figure 4.1 and 4.5 ).

For a general odd $N \geq 9$, one can decompose the Majorana channels into $N=$ $9+(N-9)$. The first 9 channels can be fractionalized by $(4.73)$, which we will discuss in detail below, and the remaining even number of channels can be split using the previous method, namely $s o(N-9)_{1}=s o\left(\frac{N-9}{2}\right)_{1} \times s o\left(\frac{N-9}{2}\right)_{1}$. In the case when $N$ is smaller than 9 , one can add $9-N$ number of non-chiral Majorana channels to each wire. These additional degrees of freedom can be interpreted as surface reconstruction as they do not violate fermion doubling[87] and are not required to live on the boundary of a topological bulk. Now each wire consists of 9 right (or left) propagating Majorana channels and $9-N$ left (resp. right) propagating ones. We still refer the remaining even channels by $s o(N-9)_{1}$ except now the negative $N-9$ signals the reverse propagating direction of these Majorana's.

The $s o(9)_{1}$ and $s o(N-9)_{1}$ sectors can then be bipartitioned independently. The fractionalization of a general odd number of Majorana channels is summarized by the sequence

$$
s o(N)_{1} \supseteq \operatorname{so}(9)_{1} \times s o(N-9)_{1} \supseteq \mathcal{G}_{N}^{+} \times \mathcal{G}_{N}^{-}
$$

for $\mathcal{G}_{N}^{ \pm}=s o(3)_{3} \times s o\left(\frac{N-9}{2}\right)_{1}$. The "+" and "-" sectors can now be back-scattered independently using (4.37) onto adjacent wires in opposite directions. This removes all low energy degrees of freedom and opens up an energy gap. 
4.2.2.1 The conformal embedding $s o(9)_{1} \supseteq s o(3)_{3}^{+} \times s o(3)_{3}^{-}$

As a matrix Lie algebra, so(3) is generated by the three anti-symmetric matrices $\Sigma=\left(\Sigma_{\mathrm{x}}, \Sigma_{\mathrm{y}}, \Sigma_{\mathrm{z}}\right)$

$$
\Sigma_{\mathrm{x}}=\left(\begin{array}{ccc}
0 & 0 & 0 \\
0 & 0 & 1 \\
0 & -1 & 0
\end{array}\right), \quad \Sigma_{\mathrm{y}}=\left(\begin{array}{ccc}
0 & 0 & 1 \\
0 & 0 & 0 \\
-1 & 0 & 0
\end{array}\right), \quad \Sigma_{\mathrm{z}}=\left(\begin{array}{ccc}
0 & 1 & 0 \\
-1 & 0 & 0 \\
0 & 0 & 0
\end{array}\right)
$$

They can be embedded into so(9) by tensoring with $\mathbb{1}_{3}$, the $3 \times 3$ identity matrix, on the left or right

$$
\Sigma^{+}=\Sigma \otimes \mathbb{1}_{3}, \quad \boldsymbol{\Sigma}^{-}=\mathbb{1}_{3} \otimes \boldsymbol{\Sigma}
$$

We denote $s o(3)^{ \pm}=\operatorname{span}\left\{\Sigma_{\mathrm{x}}^{ \pm}, \Sigma_{\mathrm{y}}^{ \pm}, \Sigma_{\mathrm{z}}^{ \pm}\right\}$to be the two mutually commuting subalgebras in $s o(9)$.

Recall the free field representation (4.8) of the $s o(9)_{1}$ WZW current generators $J^{\beta}=i \psi^{a} t_{a b}^{\beta} \psi^{b} / 2$ for $t^{\beta}$ an antisymmetric $9 \times 9$ matrix, the $s o(3)_{3}^{ \pm}$current generators are given by the substitution of $t^{\beta}$ :

$$
\mathbf{J}_{s o(3)_{3}^{ \pm}}(z)=\frac{i}{2} \psi^{a}(z) \Sigma_{a b}^{ \pm} \psi^{b}(z)
$$

for $z=e^{\tau+i x}$ and $\mathbf{J}=\left(J_{\mathbf{x}}, J_{\mathbf{y}}, J_{\mathbf{z}}\right)$. Written explicitly,

$$
\begin{array}{ll}
J_{\mathrm{x}}^{+}=i\left(\psi^{23}+\psi^{56}+\psi^{89}\right), & J_{\mathrm{x}}^{-}=i\left(\psi^{47}+\psi^{58}+\psi^{69}\right) \\
J_{\mathrm{y}}^{+}=i\left(\psi^{13}+\psi^{46}+\psi^{79}\right), & J_{\mathrm{y}}^{-}=i\left(\psi^{17}+\psi^{28}+\psi^{39}\right) \\
J_{\mathrm{z}}^{+}=i\left(\psi^{12}+\psi^{45}+\psi^{78}\right), & J_{\mathrm{z}}^{-}=i\left(\psi^{14}+\psi^{25}+\psi^{36}\right)
\end{array}
$$


for $\psi^{a b}=\psi^{a} \psi^{b}$. Using Wick's theorem and the OPE $\psi^{a}(z) \psi^{b}(w)=\delta^{a b} /(z-w)+\ldots$, it is straightforward to deduce the $s o(3)_{3}$ WZW current relations

$$
J_{\mathrm{i}}^{ \pm}(z) J_{\mathrm{j}}^{ \pm}(w)=\frac{3 \delta_{\mathrm{ij}}}{(z-w)^{2}}+\frac{i \varepsilon_{\mathrm{ijk}}}{z-w} J_{\mathrm{k}}^{ \pm}(w)+\ldots
$$

and $J_{\mathrm{i}}^{ \pm}(z) J_{\mathrm{j}}^{\mp}(w)$ is non-singular, for $\mathrm{i}, \mathrm{j}=\mathrm{x}, \mathrm{y}, \mathrm{z}$ and $\varepsilon_{\mathrm{ijk}}$ the antisymmetric tensor.

The $s o(3)_{3}$ current relations (4.77) differs from the $s o(3)_{1}$ ones $(4.10)$ by the coefficient 3 of the most singular term. This sets the level of the affine Lie algebra. The $s o(3)_{3}$ WZW theory is identical to $s u(2)_{6}$ by noticing that the structure factor of $s u(2)$ is $f_{\mathrm{ijk}}=\sqrt{2} \varepsilon_{\mathrm{ijk}}$ (see (4.68) and Ref.[26]). The $s u(2)$ current generators thus need to be normalized by $\mathbf{S}_{s u(2)_{6}^{ \pm}}=\sqrt{2} \mathbf{J}_{s o(3)}^{ \pm}$so that

$$
S_{\mathrm{i}}^{ \pm}(z) S_{\mathrm{j}}^{ \pm}(w)=\frac{6 \delta_{\mathrm{ij}}}{(z-w)^{2}}+\frac{i \sqrt{2} \varepsilon_{\mathrm{ijk}}}{z-w} S_{\mathrm{k}}^{ \pm}(w)+\ldots
$$

where the coefficient 6 of the most singular term sets the level of the $s u(2)_{6}$ affine Lie algebra.

The Sugawara energy momentum tensors are the normal ordered product

$$
T_{s o(3)_{3}^{ \pm}}(z)=\frac{1}{8} \mathbf{J}_{s o(3)_{3}^{ \pm}}(z) \cdot \mathbf{J}_{s o(3)_{3}^{ \pm}}(z)
$$

Written explicitly in the fermion representation (4.76) and using the normal ordered product

$$
\psi^{a}(z) \psi^{b}(z) \psi^{a}(z) \psi^{b}(z)=\psi^{a}(z) \partial \psi^{a}(z)+\psi^{b}(z) \partial \psi^{b}(z)
$$


the energy momentum tensor takes the form

$$
\begin{aligned}
& T_{\text {so }(3)_{3}^{ \pm}}(z)=-\frac{1}{4} \sum_{a=1}^{9} \psi^{a}(z) \partial \psi^{a}(z) \mp \frac{1}{4} \mathcal{O}_{\psi}(z) \\
& \mathcal{O}_{\psi}(z)=\psi^{1245}+\psi^{1278}+\psi^{4578}+\psi^{1346}+\psi^{1379} \\
& \quad+\psi^{4679}+\psi^{2356}+\psi^{2389}+\psi^{5689}
\end{aligned}
$$

for $\psi^{a b c d}=\psi^{a}(z) \psi^{b}(z) \psi^{c}(z) \psi^{d}(z)$. The four-fermion terms in $\mathcal{O}_{\psi}$ cancel when combining the " $\pm "$ sectors, and therefore the energy momentum tensor (4.11) completely decomposes

$$
T_{s o(9)_{1}}=-\frac{1}{2} \sum_{a=1}^{9} \psi^{a} \partial \psi^{a}=T_{s o(3)_{3}^{+}}+T_{s o(3)_{3}^{-}} .
$$

Moreover, as the OPE between $\mathbf{J}_{s o(3)_{3}^{+}}$and $\mathbf{J}_{s o(3)_{3}^{-}}$is non-singular, so is the OPE between $T_{s o(3)_{3}^{+}}$and $T_{s o(3)_{3}^{-}}$. Each sector carries half the total central charge of 9 Majorana channels

$$
c_{s o(3)_{3}^{ \pm}}=9 / 4
$$

The primary fields of $s o(3)_{3}=s u(2)_{6}$ are characterized by half-integral "angular momenta" $s=0,1 / 2, \ldots, 3 .[26]$ Each primary field $\mathbf{V}_{s}=\left(V_{s}^{-s}, V_{s}^{-s+1}, \ldots, V_{s}^{s}\right)$ irreducibly represents the WZW algebra

$$
S_{\mathrm{i}}(z) V_{s}^{m}(w)=\frac{1}{z-w} \sum_{m^{\prime}=-s}^{s}\left(S_{\mathrm{i}}^{s}\right)_{m^{\prime}}^{m} V_{s}^{m^{\prime}}(w)+\ldots
$$

for $\mathrm{i}=\mathrm{x}, \mathrm{y}, \mathrm{z}$ and $S_{\mathrm{i}}^{s}$ the $s u(2)$ generators in the spin- $s$ matrix representation. We label the seven primary fields by greek letters $\mathbf{V}_{s}=1, \alpha_{ \pm}, \gamma_{ \pm}, \beta, f$, each has conformal dimension $h_{s}=s(s+1) / 8$ (see table 4.1 ). In particular $1=\mathbf{V}_{0}$ is the vacuum and $f=\mathbf{V}_{3}$ is Abelian and fermionic with spin $3 / 2$. 


\begin{tabular}{l|lllllll}
$\mathbf{V}_{s}$ & 1 & $\alpha_{+}$ & $\gamma_{+}$ & $\beta$ & $\gamma_{-}$ & $\alpha_{-}$ & $f$ \\
\hline$s$ & 0 & $1 / 2$ & 1 & $3 / 2$ & 2 & $5 / 2$ & 3 \\
$h_{s}$ & 0 & $3 / 32$ & $1 / 4$ & $15 / 32$ & $3 / 4$ & $35 / 32$ & $3 / 2$ \\
$d_{s}$ & 1 & $\sqrt{2+\sqrt{2}}$ & $1+\sqrt{2}$ & $\sqrt{4+2 \sqrt{2}}$ & $1+\sqrt{2}$ & $\sqrt{2+\sqrt{2}}$ & 1
\end{tabular}

Table 4.1: The "angular momenta" $s$, conformal dimensions $h_{s}$ and quantum dimensions $d_{s}$ of primary fields $\mathbf{V}_{s}$ of $s o(3)_{3}=s u(2)_{6}$.

The rest of the primary fields are non-Abelian. They obey multi-channel fusion rules

$$
\mathbf{V}_{s_{1}} \times \mathbf{V}_{s_{2}}=\sum_{s} N_{s_{1} s_{2}}^{s} \mathbf{V}_{s}
$$

where the fusion matrix element $N_{s_{1} s_{2}}^{s}=0,1$ is determined by the Verlinde formula[123]

$$
N_{s_{1} s_{2}}^{s}=\sum_{s^{\prime}} \frac{\mathcal{S}_{s_{1} s^{\prime}} \mathcal{S}_{s_{2} s^{\prime}} \mathcal{S}_{s s^{\prime}}}{\mathcal{S}_{0 s^{\prime}}}
$$

and the modular $S$-matrix[26]

$$
\mathcal{S}_{s_{1} s_{2}}=\frac{1}{2} \sin \left[\frac{\pi\left(2 s_{1}+1\right)\left(2 s_{2}+1\right)}{8}\right]
$$

which is symmetric and orthogonal. Explicitly, the fusion rules are given by

$$
\begin{gathered}
f \times f=1, \quad f \times \gamma_{ \pm}=\gamma_{\mp}, \quad f \times \alpha_{ \pm}=\alpha_{\mp}, \quad f \times \beta=\beta \\
\gamma_{ \pm} \times \gamma_{ \pm}=1+\gamma_{+}+\gamma_{-}, \quad \alpha_{ \pm} \times \alpha_{ \pm}=1+\gamma_{+} \\
\beta \times \beta=1+\gamma_{+}+\gamma_{-}+f \\
\alpha_{ \pm} \times \gamma_{ \pm}=\alpha_{+}+\beta, \quad \beta \times \gamma_{ \pm}=\alpha_{+}+\alpha_{-}+\beta \\
\alpha_{ \pm} \times \beta=\gamma_{+}+\gamma_{-}
\end{gathered}
$$


The quantum dimension $d_{s}$ of the primary field $\mathbf{V}_{s}$ is defined to be the largest eigenvalue of the fusion matrix $N_{s}=\left(N_{s s_{1}}^{s_{2}}\right)$. It coincides with the modular $S$ matrix element $\mathcal{S}_{0 s} / \mathcal{S}_{00}$ and respects fusion rules so that

$$
d_{s_{1}} d_{s_{2}}=\sum_{s} N_{s_{1} s_{2}}^{s} d_{s}
$$

They are listed in table 4.1 .

\subsubsection{2 $\mathbb{Z}_{6}$ parafermions}

We first study the simplest odd case when there are 9 Majorana cones mimicked by the coupled Majorana wire model (4.3) with 9 chiral Majorana channels per wire. Now that we have bipartite the degrees of freedom according to the two $s o(3)_{3}^{ \pm} \mathrm{WZW}$ current algebras in (4.76), they can be backscattered independently to adjacent wires in opposite directions (see eq.(4.37) and figure 4.1). As the so(3) ${ }_{3}^{+}$sector completely decomposes from the $s o(3)_{3}^{-}$one, the current backscattering $\mathbf{J}_{s o(3)_{3}^{-}}^{y-1} \cdot \mathbf{J}_{s o(3)_{3}^{+}}^{y}$ between the $(y-1)^{\text {th }}$ and $y^{\text {th }}$ wire does not compete with the next pair $\mathbf{J}_{s o(3)_{3}^{-}}^{y} \cdot \mathbf{J}_{s o(3)_{3}^{+}}^{y+1}$.

The current-current interaction consists of four-fermion terms and is marginally relevant. This can be seen from the RG equation (4.45) using the operator product expansion $\left(\mathbf{J}^{y} \cdot \mathbf{J}^{y+1}\right)^{2} \sim+\mathbf{J}^{y} \cdot \mathbf{J}^{y+1}$. (Recall the time reversal symmetric convention (4.40) and that $\mathbf{J}^{y} \mathbf{J}^{y} \sim i(-1)^{y} \mathbf{J}^{y}$.) To see that the interaction indeed opens up an excitation energy gap, it suffices to focus on a single pair of wires with the Hamiltonian

$$
\mathcal{H}_{\text {int }}=u \mathbf{J}_{s o(3)_{3}^{-}}^{R} \cdot \mathbf{J}_{s o(3)_{3}^{+}}^{L}
$$

where $R / L$ labels the counter-propagating directions along wire $y$ and $y+1$.

First we further decompose the $s o(3)_{3}$ WZW theory by the coset construction[26]

$$
s o(3)_{3}=u(1)_{6} \times “ \mathbb{Z}_{6} ", \quad " \mathbb{Z}_{6} "=\frac{s o(3)_{3}}{s o(2)_{3}}=\frac{s u(2)_{6}}{u(1)_{6}}
$$


where " $\mathbb{Z}_{6}$ " refers to the $\mathbb{Z}_{6}$ parafermion CFT model by Zamolodchikov and Fateev[29, 144]. This is done by noticing that $S O(3)$ (or equivalently $S U(2)$ ) contains the Abelian subgroup $S O(2)$ (resp. $U(1)$ ) of rotations about the z-axis, and on the CFT level, the $s o(2)_{3}$ WZW sub-theory of $s o(3)_{3}$ (resp. $\left.u(1)_{6} \subseteq s u(2)_{6}\right)$ can be bosonized and single-out. To do this we first group three pairs of Majorana fermions into three Dirac fermions on each chiral sector

$$
\begin{aligned}
& c_{R}^{1}=\frac{\psi_{R}^{1}+i \psi_{R}^{4}}{\sqrt{2}}, \quad c_{R}^{2}=\frac{\psi_{R}^{2}+i \psi_{R}^{5}}{\sqrt{2}}, \quad c_{R}^{3}=\frac{\psi_{R}^{3}+i \psi_{R}^{6}}{\sqrt{2}} \\
& c_{L}^{1}=\frac{\psi_{L}^{1}+i \psi_{L}^{2}}{\sqrt{2}}, \quad c_{L}^{2}=\frac{\psi_{R}^{4}+i \psi_{L}^{5}}{\sqrt{2}}, \quad c_{L}^{3}=\frac{\psi_{L}^{7}+i \psi_{L}^{8}}{\sqrt{2}}
\end{aligned}
$$

and bosonize

$$
c_{R / L}^{j} \sim \frac{1}{\sqrt{l}_{0}} \exp \left(i \widetilde{\phi}_{R / L}^{j}\right)
$$

for $j=1,2,3$. The $s o(2)_{3}$ subalgebra in the $R$ and $L$ sectors are generated by the $J_{z}^{-}$ and $J_{\mathbf{z}}^{+}$currents operators in $(4.76)$

$$
J_{\mathrm{z}}^{R}=-3 i \partial \phi_{R}^{\rho}, \quad J_{\mathrm{z}}^{L}=3 i \partial \phi_{L}^{\rho}
$$

where the boson field of the "charge" sector is the average

$$
\phi_{R / L}^{\rho}=\frac{\widetilde{\phi}_{R / L}^{1}+\widetilde{\phi}_{R / L}^{2}+\widetilde{\phi}_{R / L}^{3}}{3} .
$$

The "neutral" sector is carried by the three boson fields

$$
\phi_{R / L}^{\sigma, j}=\widetilde{\phi}_{R / L}^{j}-\phi_{R / L}^{\rho}
$$

which are not independent as $\phi^{\sigma, 1}+\phi^{\sigma, 2}+\phi^{\sigma, 3}=0$. 
It is straightforward to check that the "charge" and the "neutral" sectors completely decouple from each other. For instance, the Lagrangian density decomposes

$$
\begin{aligned}
\mathcal{L}_{R / L} & =\frac{(-1)^{R / L}}{2 \pi} \sum_{j=1}^{3} \partial_{x} \widetilde{\phi}_{R / L}^{j} \partial_{t} \widetilde{\phi}_{R / L}^{j} \\
& =\frac{(-1)^{R / L}}{2 \pi}\left[3 \partial_{x} \phi_{R / L}^{\rho} \partial_{t} \phi_{R / L}^{\rho}+\sum_{j=1}^{3} \partial_{x} \phi_{R / L}^{\sigma, j} \partial_{t} \phi_{R / L}^{\sigma, j}\right]
\end{aligned}
$$

where the remaining fermions $\psi_{R}^{7,8,9}, \psi_{L}^{3,6,9}$ are suppressed, and $(-1)^{R}=1,(-1)^{L}=$ $-1$

The Lagrangian density (4.97) involves more degrees of freedom in $s o(9)_{1}^{R / L}$ than just $s o(3)_{3}^{R,-}$ or $s o(3)_{3}^{L,+}$. Therefore, a priori, it is not obvious that this $\rho-\sigma$ decomposition is a splitting of $s o(3)_{3}$, and in fact it is not. Only the charge sector $\phi_{R / L}^{\rho}$ is entirely belonging to $s o(3)_{3}^{R,-}$ or $s o(3)_{3}^{L,+}$. To show this, we go back to the energy-momentum tensor $T_{s o(3)_{3}^{ \pm}}$in (4.81), say for $R$ movers.

$$
T_{s o(3)_{3}^{R, \pm}}(z)=\frac{1}{2} T_{s o(9)_{1}^{R}}(z) \mp \frac{1}{4} \mathcal{O}_{\psi}(z)
$$

where the total energy-momentum tensor in partially bosonized basis is

$$
\begin{aligned}
T_{s o(9)_{1}^{R}}=- & \frac{1}{2}\left[3 \partial \phi_{R}^{\rho} \partial \phi_{R}^{\rho}+\sum_{j=1}^{3} \partial \phi_{R}^{\sigma, j} \partial \phi_{R}^{\sigma, j}\right. \\
& \left.+\psi_{R}^{7} \partial \psi_{R}^{7}+\psi_{R}^{8} \partial \psi_{R}^{8}+\psi_{R}^{9} \partial \psi_{R}^{9}\right]
\end{aligned}
$$

and the operator $\mathcal{O}_{\psi}$ defined in (4.82) is now

$$
\begin{aligned}
& \mathcal{O}_{\psi}=-3 \partial \phi_{R}^{\rho} \partial \phi_{R}^{\rho}+\frac{1}{2} \sum_{j=1}^{3} \partial \phi_{R}^{\sigma, j} \partial \phi_{R}^{\sigma, j} \\
&-2 i\left[\cos \left(\phi_{R}^{\sigma, 1}-\phi_{R}^{\sigma, 2}\right) \psi_{R}^{78}+\cos \left(\phi_{R}^{\sigma, 1}-\phi_{R}^{\sigma, 3}\right) \psi_{R}^{97}\right. \\
&\left.\quad+\cos \left(\phi_{R}^{\sigma, 2}-\phi_{R}^{\sigma, 3}\right) \psi_{R}^{89}\right]
\end{aligned}
$$


Eq.(4.100) is deduced by substituting the fermions by the boson fields (4.93), whose OPE can be found in (D.1,D.2,D.3) in appendix D. For instance, the factor of $i$ in (4.100) is a result of mutually non-commuting $\phi^{\sigma, j}$. More importantly, $\phi^{\rho}, \phi^{\sigma}$ and $\psi^{7,8,9}$ are completely decoupled. As the "charge" sector $\phi_{R}^{\rho}$ only appears in $T_{s o(3)_{3}^{R,-}}$, it belongs entirely in $s o(3)_{3}^{R,-}$. Similarly $\phi_{L}^{\rho}$ belongs entirely in $s o(3)_{3}^{L,+}$. The " $\mathbb{Z}_{6}$ " energy-momentum is defined by subtracting the decoupled "charge" sector from $\mathrm{so}(3)_{3}$.

$$
\begin{aligned}
T_{s o(2)_{3}^{R}}= & \frac{1}{6} J_{\mathrm{z}} J_{\mathrm{z}}=-\frac{1}{2} 3 \partial \phi_{\rho} \partial \phi_{\rho} \\
T_{\mathbb{Z}_{6}}^{R}= & T_{s o(3)_{3}^{R,-}}-T_{s o(2)_{3}^{R}} \\
= & -\frac{1}{4} \sum_{a=7}^{9} \psi_{R}^{a} \partial \psi_{R}^{a}-\frac{1}{8} \sum_{j=1}^{3} \partial \phi_{R}^{\sigma, j} \partial \phi_{R}^{\sigma, j} \\
& -\frac{i}{2}\left[\cos \left(\phi_{R}^{\sigma, 1}-\phi_{R}^{\sigma, 2}\right) \psi_{R}^{78}+\cos \left(\phi_{R}^{\sigma, 1}-\phi_{R}^{\sigma, 3}\right) \psi_{R}^{97}\right. \\
& \left.+\cos \left(\phi_{R}^{\sigma, 2}-\phi_{R}^{\sigma, 3}\right) \psi_{R}^{89}\right]
\end{aligned}
$$

and similarly for the $L$ movers.

The remaining current operators $J_{ \pm}=\left(J_{\mathrm{x}} \pm i J_{\mathrm{y}}\right) / \sqrt{2}$ of $s o(3)_{3}^{-}$in the $R$ sector and $s o(3)_{3}^{+}$in the $L$ sector (see eq.(4.76)) now split into "charge" and "neutrual" parafermion components

$$
J_{ \pm}^{R / L}=\mp \sqrt{3} e^{\mp i \phi_{R / L}^{\rho}} \Psi_{R / L}^{\mp}
$$

where the $\mathbb{Z}_{6}$ parafermions are given by the combinations

$$
\begin{aligned}
& \Psi_{R}=\frac{1}{\sqrt{3}}\left(e^{i \phi_{R}^{\sigma, 1}} \psi_{R}^{7}+e^{i \phi_{R}^{\sigma, 2}} \psi_{R}^{8}+e^{i \phi_{R}^{\sigma, 3}} \psi_{R}^{9}\right) \\
& \Psi_{L}=\frac{1}{\sqrt{3}}\left(e^{i \phi_{L}^{\sigma, 1}} \psi_{L}^{3}+e^{i \phi_{L}^{\sigma, 2}} \psi_{L}^{6}+e^{i \phi_{L}^{\sigma, 3}} \psi_{L}^{9}\right)
\end{aligned}
$$


for $\Psi_{R / L}^{+}=\Psi_{R / L}$ and $\Psi_{R / L}^{-}=\Psi_{R / L}^{\dagger}$. Unlike the $\phi^{\sigma}$ 's, here the "neutral" $\mathbb{Z}_{6}$ parafermions $\Psi_{R / L}$ belongs entirely in $s o(3)_{3}^{R,-}$ or $s o(3)_{3}^{L,+}$. This is because $\mathbf{J}^{R / L}$ and $\phi_{R / L}^{\rho}$ both completely sit inside the $s o(3)_{3}$ 's as seen above. Otherwise one can verified this by computing the OPE with the energy-momentum tensor (4.99) explicitly

$$
\begin{aligned}
T_{s o(3)_{3}^{R,-}}(z) \Psi_{R}(w) & =\frac{5 / 6}{(z-w)^{2}} \Psi_{R}(w)+\frac{\partial \Psi_{R}(w)}{z-w}+\ldots \\
T_{s o(3)_{3}^{R,-}}(z) e^{ \pm i \phi_{R}^{\rho}(w)} & =\frac{1 / 6}{(z-w)^{2}} e^{ \pm i \phi_{R}^{\rho}(w)}+\frac{\partial e^{ \pm i \phi_{R}^{\rho}(w)}}{z-w}+\ldots
\end{aligned}
$$

and $T_{s o(3)_{3}^{R,+}}(z) \Psi_{R}(w)$ and $T_{s o(3)_{3}^{R,+}}(z) e^{ \pm i \phi_{R}^{\rho}(w)}$ are both non-singular. Similar OPE hold for the $L$ sector. The primary fields (4.104) generate the rest of the $\mathbb{Z}_{6}$ parafermions (see (D.5) in appendix D) and they obey the known $\mathbb{Z}_{6}$ structure by Zamolodchikov and Fateev[144].

\subsubsection{Gapping potential}

Now that we have further decomposed the $s o(3)_{3}^{ \pm}$currents in each wire into $s o(2)_{3}=U(1)_{6}$ and $\mathbb{Z}_{6}$ parafermion components (see eq.(4.103)), the current-current backscattering interaction (4.91) between a pair of wires takes the form of

$$
\mathcal{H}_{\text {int }}=9 u \partial_{x} \phi_{R}^{\rho} \partial_{x} \phi_{L}^{\rho}+3 u\left[e^{i\left(\phi_{L}^{\rho}-\phi_{R}^{\rho}\right)} \Psi_{R}^{\dagger} \Psi_{L}+\text { h.c. }\right]
$$

The first term only renormalizes the velocity of the boson in the $s o(2)_{3}$ sector. The second term is responsible for openning an excitation energy gap. It extracts a $\mathbb{Z}_{6}$ parafermion $\Psi$ and a quasiparticle $e^{i \phi^{\rho}}$ from the $s o(3)_{3}^{+}$sector on the $y^{\text {th }}$ wire and backscatter them onto the $s o(3)_{3}^{-}$sector along the $(y+1)^{t h}$ wire. This freezes all low energy degrees of freedom and the ground state is characterized by the $\mathbb{Z}_{6}$ expectation 
value $(\mathrm{GEV})$

$$
\left\langle\Psi_{R}^{\dagger}(x) \Psi_{L}(x)\right\rangle \sim-e^{i\left\langle\phi_{R}^{\rho}(x)-\phi_{L}^{\rho}(x)\right\rangle}=e^{2 \pi i m / 6}
$$

for $m$ an integer.

Like the $O(N)$ Gross-Neveu model we discussed in section 4.2.1.1, quasiparticle excitations here also manifest as kinks or domain walls between segments with different GEV's. The primary fields $\alpha_{ \pm}, \gamma_{ \pm}, \beta$ of the chiral so $(3)_{3}$ WZW theory in table 4.1 decompose into components in the " $\mathbb{Z}_{6}$ " and $s o(2)_{3}$ sectors.

$$
\begin{gathered}
\alpha_{+}=\left[\sigma_{1}\right] \times\left[e^{i \phi^{\rho} / 2}\right], \quad \alpha_{-}=\left[\sigma_{5}\right] \times\left[e^{-i \phi^{\rho} / 2}\right] \\
\gamma_{+}=\left[\sigma_{2}\right] \times\left[e^{i \phi^{\rho}}\right], \quad \gamma_{-}=\left[\sigma_{4}\right] \times\left[e^{-i \phi^{\rho}}\right] \\
\beta=\left[\sigma_{3}\right] \times\left[e^{i 3 \phi^{\rho} / 2}\right]
\end{gathered}
$$

where $\sigma_{l}$ are primary fields in the chiral $\mathbb{Z}_{6}$ parafermion theory so that $\sigma_{l}^{R} \sigma_{l}^{L}$ take the roles of the order parameters of the $\mathbb{Z}_{6} \operatorname{model}[29,144]$. They satisfy the exchange relations

$$
\Psi(x) \sigma_{l}\left(x^{\prime}\right)=\sigma_{l}\left(x^{\prime}\right) \Psi(x) e^{-2 \pi i \frac{l}{6} \theta\left(x-x^{\prime}\right)}
$$

for $R$ sector, and similar relations hold for the $L$ sector with the $\mathbb{Z}_{6}$ phases conjugated. Therefore adding the operators $\alpha_{ \pm}(x), \gamma_{ \pm}(x), \beta(x)$ to the ground state create kinks of different hights in the GEV (4.107)

$$
\begin{aligned}
& \left\langle\alpha_{ \pm}^{\dagger}\left(x_{0}\right) \Psi_{R}^{\dagger}(x) \Psi_{L}(x) \alpha_{ \pm}\left(x_{0}\right)\right\rangle \sim e^{\frac{\pi i}{3}\left(m \pm \theta\left(x-x_{0}\right)\right)} \\
& \left\langle\gamma_{ \pm}^{\dagger}\left(x_{0}\right) \Psi_{R}^{\dagger}(x) \Psi_{L}(x) \gamma_{ \pm}\left(x_{0}\right)\right\rangle \sim e^{\frac{\pi i}{3}\left(m \pm 2 \theta\left(x-x_{0}\right)\right)} \\
& \left\langle\beta^{\dagger}\left(x_{0}\right) \Psi_{R}^{\dagger}(x) \Psi_{L}(x) \beta\left(x_{0}\right)\right\rangle \sim e^{\frac{\pi i}{3}\left(m+3 \theta\left(x-x_{0}\right)\right)}
\end{aligned}
$$


where $\theta(s)=(s /|s|+1) / 2$ is the unit step function.

The fermionic supersector $f$ in $s o(3)_{3}$ (see table 4.1) consists of operators that admit free field representations. Again we focus on the $s o(3)_{3}^{R,-}$ sector. The operators

$$
\begin{gathered}
V_{f}^{0}=\Psi^{3}, \quad V_{f}^{ \pm 1}=e^{\mp i \phi^{\rho}} \Psi^{\mp 2} \\
V_{f}^{ \pm 2}=e^{\mp 2 i \phi^{\rho}} \Psi^{\mp}, \quad V_{f}^{ \pm 3}=e^{\mp 3 i \phi^{\rho}}
\end{gathered}
$$

span a $s=3$ representation of the affine $s o(3)_{3}$ Lie algebra, where $\Psi^{-m}=\Psi^{6-m}$ are the $\mathbb{Z}_{6}$ parafermions satisfying the $\mathrm{OPE} \Psi^{m}(z) \Psi^{m^{\prime}}(w) \sim(z-w)^{-m m^{\prime} / 3} \Psi^{m+m^{\prime}}$ (see appendix D for explicit definitions). From (4.109), they create a kink to the order parameter $\langle b\rangle=\left\langle\beta_{R}(x) \beta_{L}(x)\right\rangle$

$$
\left\langle\mathbf{V}_{f}^{R}\left(x_{0}\right)^{\dagger} \beta_{R}(x) \beta_{L}(x) \mathbf{V}_{f}^{R}\left(x_{0}\right)\right\rangle=\langle b\rangle(-1)^{\theta\left(x-x_{0}\right)}
$$

in the order phase.

The gapping potential can now be generalized to an arbitrary odd number of Majorana channels per wire. Using the decomposition (4.74), the $N$ Majorana channels are first split into $9+(N-9)$. The first 9 channels are fractionalized into $s o(3)_{3}^{+} \times s o(3)_{3}^{-}$ while the remaining $N-9$ can be split into $s o\left(\frac{N-9}{2}\right)_{1}^{+} \times s o\left(\frac{N-9}{2}\right)_{1}^{-}$because $N-9$ is even. The interwire current backscattering (4.37) takes the form

$$
\mathcal{H}_{\mathrm{int}}=u \sum_{y=-\infty}^{\infty} \mathbf{J}_{s o(3)_{3}^{-}}^{y} \cdot \mathbf{J}_{s o(3)_{3}^{+}}^{y+1}+\mathbf{J}_{s o\left(\frac{N-9}{2}\right)_{1}^{-}} \cdot \mathbf{J}_{s o\left(\frac{N-9}{2}\right)_{1}^{+}}^{y+1}
$$

where different terms act on completely decoupled degrees of freedom. They also gap out all low energy degrees freedom as the energy-momentum tensor of the CFT along 
each wire decomposes

$$
\begin{aligned}
T_{s o(N)_{1}} & =T_{s o(9)_{1}}+T_{s o(N-9)_{1}} \\
& =T_{s o(3)_{3}^{+}}+T_{s o(3)_{3}^{-}}+T_{s o\left(\frac{N-9}{2}\right)_{1}^{+}}+T_{s o\left(\frac{N-9}{2}\right)_{1}^{-}}
\end{aligned}
$$

using (4.83) and the fact that

$$
T_{s o(m+n)_{1}}=-\frac{1}{2} \sum_{a=1}^{m+n} \psi^{a} \partial \psi^{a}=T_{s o(m)_{1}}+T_{s o(n)_{1}}
$$

\subsubsection{Gapping by fractional quantum Hall stripes}
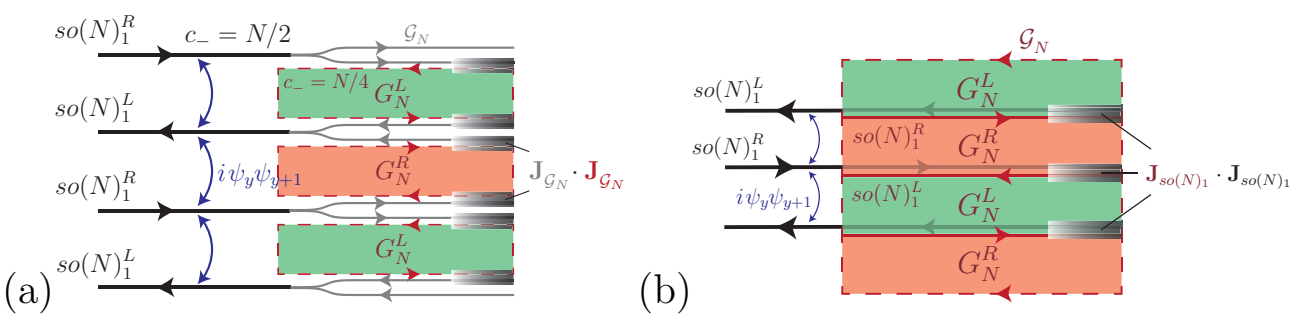

Figure 4.6: Gapping $N$ surface Majorana cones by inserting $(2+1) \mathrm{D} G_{N}$ stripe state and removing edge modes by current-current backscattering interaction.

Previously, we designed interwire interactions that gap all Majorana modes without breaking time reversal symmetry. Here we provide an alternative where each chiral Majorana wire is gapped by backscattering onto the edges of two topological stripes sandwiching the wire (see figure 4.6). The topological stripes could be fractional quantum Hall states for instance. Similar construction has been proposed to describe surface states of topological insulators[81].

First we consider inserting between each pairs of Majorana wire a $(2+1)$ D topological state. It supports chiral boundary modes which move in a reverse direction to its neighboring Majorana wire. As adjacent wires have opposite propagation directions, the chiralities of the topological states also alternates. This alternating topological stripe state can be regarded as a surface reconstruction of the 3D topological super- 
conductor. It preserves the antiferrormagnetic time reversal symmetry (4.1), which relates adjacent topological stripes by reversing their chirality. Unlike the coupled Majorana wire mode, the topological stripe state itself is a pure $(2+1) \mathrm{D}$ time reversal symmetric system and is not supported by a $(3+1) \mathrm{D}$ bulk. It has a gapless energy spectrum that is identical to $N$ surface Majorana cones and is carried by the interface modes between stripes (see figure 4.6(b)). However the topological stripe state also carry non-trivial anyonic excitations between wires. This distinguishes it from the coupled Majorana wire model and allows it to exist non-holographically in a pure $(2+1) \mathrm{D}$ setting.

The Majorana modes along the chiral wires then can be backscattered onto the boundaries or interfaces of the topological stripes by current-current couplings. In order for the boundary or interface modes to exactly cancel the Majorana modes along each wire, the topological stripes must carry specific topological orders. We take a $G_{N}$ topological state

$$
G_{N}= \begin{cases}S O(r)_{1}, & \text { for } N=2 r \\ S O(3)_{3} \otimes_{b} S O(r)_{1}, & \text { for } N=9+2 r\end{cases}
$$

so that its boundary carries a $\mathcal{G}_{N}$ Kac-Moody current, for $\mathcal{G}_{N}$ the affine Lie algebra of $G_{N}$ defined in $(4.34) . G_{N}^{R}$ and $G_{N}^{L}$ denote stripes with opposite chiralities. The $(2+$ 1)D $G_{N}$ topological state itself can be constructed using a coupled wire construction similar to that in Ref.[117, 58] and will not be discussed here.

There are two ways the Majorana modes can be backscattered onto the topological stripes. The first is shown in figure 4.6(a). The $N$ Majorana modes along each chiral wire is bipartite into a pair of WZW theories $\mathcal{G}_{N}^{+} \times \mathcal{G}_{N}^{-}$according to (4.33). Each WZW theory is identical to the CFT along the boundary of an neighboring topological stripe but propagates in an opposite direction. It can be then be gapped out by the current- 
current backscattering

$$
\mathcal{H}_{\text {int }}=u \mathbf{J}_{\mathcal{G}_{N}}^{\text {wire }} \cdot \mathbf{J}_{\mathcal{G}_{N}}^{\text {stripe }}
$$

Alternatively, one could first glue the topological stripes together (see figure 4.6(b)) so that the line interface sandwiched between adjacent $G_{N}^{R}$ and $G_{N}^{L}$ states hosts a chiral $s o(N)_{1}$ CFT. The stripes can then be put on top of the Majorana wire array so that each interface is sitting on top of a wire with opposite chirality. The current-current backscattering

$$
\mathcal{H}_{\text {int }}=u \mathbf{J}_{s o(N)_{1}}^{\text {wire }} \cdot \mathbf{J}_{s o(N)_{1}}^{\text {interface }}
$$

between each Majorana wire and stripe interface gaps out all low energy degrees of freedom.

\subsection{Surface topological order}

In the previous section, we described how a coupled Majorana wire model, which mimics the surface Majorana modes of a 3D bulk topological superconductor (TSC), can be gapped by interwire current-current backscattering interaction without breaking time reversal (TR) symmetry. In this section, we pay more attention to the topological order and the anyon types[137, 36, 134] of gapped excitations. The ground states are time reversal symmetric and there are no non-vanishing order parameters that breaks time reversal spontaneously. There is a finite ground state degeneracy that does not depend on system size. This signifies a non-trivial topological $\operatorname{order}[131,132,135]$.

The surface topological order can be inferred from bulk-boundary correspondence[133, 98, 99, 60]. There is a one-to-one correspondence between the primary fields of the 


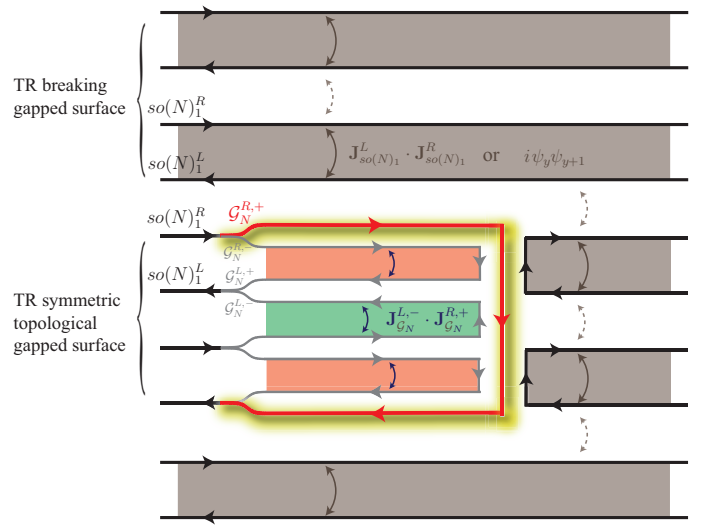

Figure 4.7: Chiral interface (highlighted line) between a time reversal breaking gapped region and a TR symmetric topologically ordered gapped region.

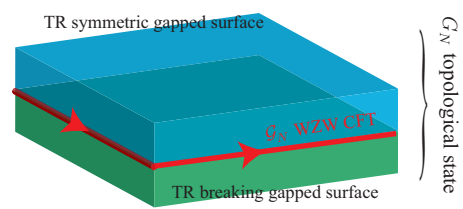

Figure 4.8: The $G_{N}$ topological order of a quasi-2D slab with time reversal symmetric gapped top surface and time reversal breaking gapped bottom surface

CFT along the $(1+1) \mathrm{D}$ gapless boundary and the anyon types in the $(2+1) \mathrm{D}$ gapped topological bulk. The conformal scaling dimension or spin $h=h_{R}-h_{L}$ of a primary field corresponds to the exchange statistical phase $\theta=e^{2 \pi i h}$ of the corresponding anyon. The fusion rules of primary fields are identical to that of the anyons. And the modular $S$-matrix of the CFT at the boundary equals the braiding $S$-matrix[60]

$$
\mathcal{S}_{\mathbf{a b}}=\frac{1}{\mathcal{D}} \sum_{\mathbf{c}} d_{\mathbf{c}} N_{\mathbf{a b}}^{\mathbf{c}} \frac{\theta_{\mathbf{c}}}{\theta_{\mathbf{a}} \theta_{\mathbf{b}}}
$$

in the bulk, where the non-negative integers $N_{\mathbf{a b}}^{\mathbf{c}}$ are the degeneracies of the fusion rules

$$
\mathbf{a} \times \mathbf{b}=\sum_{\mathbf{c}} N_{\mathbf{a b}}^{\mathbf{c}} \mathbf{c}
$$


between anyons, and the total quantum dimension $\mathcal{D}=\sqrt{\sum_{\mathbf{a}} d_{\mathbf{a}}^{2}}$ quantifies topological entanglement[63] and can be evaluated by knowing the quantum dimensions $d_{\mathbf{a}} \geq 1$ of each anyon a by solving the fusion identities

$$
d_{\mathbf{a}} d_{\mathbf{b}}=\sum_{\mathbf{c}} N_{\mathbf{a b}}^{\mathbf{c}} d_{\mathbf{c}}
$$

On the surface of a topological superconductor, where there are no boundaries, the $(2+1) \mathrm{D}$ topological order corresponds to a $(1+1) \mathrm{D}$ interface that separate the time reversal symmetric topologically ordered domain and a time reversal breaking domain. This interface hosts chiral gapless modes (see figure 4.7). This geometry can be wrapped onto the surface of a slab where the TR symmetric and breaking domains occupy the top and bottom surface of a 3D bulk (see figure 4.8). The quasi-2D system has an energy gap except along its boundary which is previously the interface that carries the $\mathcal{G}_{N}$ WZW CFT. The bulk-boundary correspondence then determines a bulk $G_{N}$ topological order on the quasi-2D slab.

Wires in the trivial TR-breaking domain are gapped by non-uniform current backscattering

$$
\begin{array}{r}
\mathcal{H}_{\mathrm{TR}-\text { breaking }}=\sum_{y} \Delta \mathbf{J}_{s o(N)_{1}}^{2 y-1} \cdot \mathbf{J}_{s o(N)_{1}}^{2 y} \\
+\delta \mathbf{J}_{s o(N)_{1}}^{2 y} \cdot \mathbf{J}_{s o(N)_{1}}^{2 y+1}
\end{array}
$$

or single-body fermion backscattering perturbation

$$
\mathcal{H}_{\mathrm{TR}-\text { breaking }}=\sum_{y} i \Delta \boldsymbol{\psi}_{2 y-1}^{T} \boldsymbol{\psi}_{2 y}+i \delta \boldsymbol{\psi}_{2 y}^{T} \boldsymbol{\psi}_{2 y+1}
$$

to the coupled Majorana wire model (4.3), for $\Delta>\delta$ and $\boldsymbol{\psi}_{y}=\left(\psi_{y}^{1}, \ldots, \psi_{y}^{N}\right)$. This violates the antiferrormagnetic time reversal symmetry (4.1) and leads to a gapped 
surface with trivial topological order. This TR breaking half-plane is put side by side against a TR symmetric gapped half-plane, where the $N$ Majorana channels per wire is fractionalized into $s o(N)_{1} \supseteq \mathcal{G}_{N}^{+} \times \mathcal{G}_{N}^{-}$, for $\mathcal{G}_{N}$ previously defined in (4.34). Each $\mathcal{G}_{N}$ sector is then paired with the adjacent one on the next wire and are gapped by current-current backscattering $\mathbf{J}_{\mathcal{G}_{N}^{-}} \cdot \mathbf{J}_{\mathcal{G}_{N}^{+}}$. The interface between the TR-symmetric and TR-breaking regions leaves behind one single unpaired fractional $\mathcal{G}_{N}$ channel. This can be regarded as a 2D analogue of the fractional boundary modes in the Haldane integral spin chain[44, 45] and the AKLT spin chain[1].

As eluded in the introduction, when the coupled wire model involves only currentcurrent backscattering interaction, it is a boson model where the bosonic current operators, rather than Majorana fermions, are treated as fundamental local objects. It is therefore more natural for us to use the current backscattering Hamiltonian (4.121) instead of the fermionic single-body one (4.122) to introduce a time reversal breaking gap. In this case, $\pi$-fluxes are deconfined anyonic excitations realized as $\pi$-kinks along a stripe where there is no energy cost in separating a flux-antiflux pair. If the fermionic TR-breaking Hamiltonian (4.122) were used instead, $\pi$-fluxes would be confined on the bottom layer and Majorana fermions would become local. We however will mostly be focusing on the former bosonic case, although the fermionic scenario may be more realistic in a superconducting medium.

The bulk-interface correspondence depends on the orientation of the time reversal breaking order. In eq.(4.121), if the backscattering tunneling strengths are reversed so that $\delta>\Delta$, figure 4.7 will need to be shifted by $y \rightarrow y+1$ and all propagating directions will need to be inverted. As a result, the interface CFT will also be reversed to its time reversal partner $\mathcal{G}_{N} \rightarrow \overline{\mathcal{G}_{N}}$. This will flip the spins of all primary fields $h_{\mathbf{a}} \rightarrow h_{\overline{\mathbf{a}}}=-h_{\mathbf{a}}$ and conjugates all exchange phases $\theta_{\mathbf{a}} \rightarrow \theta_{\overline{\mathbf{a}}}=\theta_{\mathbf{a}}^{*}$.

An interface with a particular orientation therefore corresponds to a time reversal breaking topological order. This is also apparent in the slab geometry in figure 4.8 
where the TR breaking order on the bottom surface can have opposite orientations. Unlike the conventional case on the surface of a topological superconductor where time reversal is local, here time reversal involves a half translation $y \rightarrow y+1$ and relates a stripe gapped by $\mathbf{J}_{y}^{-} \cdot \mathbf{J}_{y+1}^{+}$to its neighbor $\mathbf{J}_{y+1}^{-} \cdot \mathbf{J}_{y+2}^{+}$. As anyonic excitations are realized as kinks or domain walls that separate distinct ground states along a stripe, time reversal non-locally translates anyons on an even stripe (green) to an odd one (red) or vice versa (see figure 4.7). However an interface with a particular orientation can only correspond to anyons on stripes with a particular parity. For example the bulk-interface correspondence in figure 4.7 singles out anyons on even stripes gapped by $\mathbf{J}_{2 y}^{-} \cdot \mathbf{J}_{2 y+1}^{+}$. There is therefore no reason to expect the anyon theory would be closed under time reversal.

\subsubsection{Summary of anyon contents}

\begin{tabular}{l|llll|lll} 
& \multicolumn{5}{|c|}{$r$ even } & \multicolumn{3}{c}{$r$ odd } \\
$\mathbf{x}$ & 1 & $\psi$ & $s_{+}$ & $s_{-}$ & 1 & $\psi$ & $\sigma$ \\
\hline$d_{\mathbf{x}}$ & 1 & 1 & 1 & 1 & 1 & 1 & $\sqrt{2}$ \\
$\theta_{\mathbf{x}}$ & 1 & -1 & $e^{\pi i r / 8}$ & $e^{\pi i r / 8}$ & 1 & -1 & $e^{\pi i r / 8}$
\end{tabular}

Table 4.2: The exchange phase $\theta_{\mathbf{x}}=e^{2 \pi i h_{\mathbf{x}}}$ and quantum dimensions of anyons $\mathbf{x}$ in a $(2+1) \mathrm{D} S O(r)_{1}$ topological phase.

The interface carries chiral gapless degrees of freedom, which are captured by the $\mathcal{G}_{N}$ WZW theory whose primary fields corresponds to the anyon content of the TR symmetry gapped surface. For even $N=2 r$, the surface carries a

$$
G_{N}=S O(r)_{1}
$$


topological order summarized in table 4.2. Its anyonic excitations obey the abelian fusion rules

$$
\begin{gathered}
\psi \times \psi=1, \quad s_{ \pm} \times \psi=s_{\mp} \\
s_{ \pm} \times s_{ \pm}=\left\{\begin{array}{rr}
1, & \text { for } r \equiv 0 \bmod 4 \\
\psi, & \text { for } r \equiv 2 \bmod 4
\end{array}\right.
\end{gathered}
$$

for $r$ even, or the Ising fusion rules

$$
\psi \times \psi=1, \quad \psi \times \sigma=\sigma, \quad \sigma \times \sigma=1+\psi
$$

for $r$ odd. Eq.(4.124) and (4.125) follows directly from the fusion properties of the primary fields in the $s o(r)_{1}$ Kac-Moody algebra (see section 4.1 .1 and appendix B and $\mathrm{C}$ ). The exchange phase (also known as topological spin) $\theta_{\mathbf{x}}=e^{2 \pi i h_{\mathbf{x}}}$ can be read off from the conformal dimension $h_{\mathbf{x}}$ of the primary field $V_{\mathbf{x}}$ in $s o(r)_{1}$ that corresponds to the anyon type $\mathbf{x}$. Again we extend $r$ to negative integers by defining $S O(-r)_{1}=\overline{S O(r)_{1}}$ to be the time reversal conjugate of the $S O(r)_{1}$ topological state.

\begin{tabular}{l|lllllll}
$\mathbf{x}$ & 1 & $\alpha_{+}$ & $\gamma_{+}$ & $\beta$ & $\gamma_{-}$ & $\alpha_{-}$ & $f$ \\
\hline$d_{\mathbf{x}}$ & 1 & $\sqrt{2+\sqrt{2}}$ & $1+\sqrt{2}$ & $\sqrt{4+2 \sqrt{2}}$ & $1+\sqrt{2}$ & $\sqrt{2+\sqrt{2}}$ & 1 \\
$\theta_{\mathbf{x}}$ & 1 & $e^{\pi i \frac{3+2 r}{16}}$ & $e^{i \pi / 2}$ & $e^{\pi i \frac{15+2 r}{16}}$ & $e^{-i \pi / 2}$ & $e^{\pi i \frac{3+2 r}{16}}$ & -1 \\
$\mathbf{x}$ & 1 & $\alpha_{+}$ & $\gamma_{+}$ & $\beta$ & & & \\
\hline$d_{\mathbf{x}}$ & 1 & $\sqrt{2+\sqrt{2}}$ & $1+\sqrt{2}$ & $\sqrt{4+2 \sqrt{2}}$ & $1+\sqrt{2}$ & $\sqrt{2+\sqrt{2}}$ & 1 \\
$\theta_{\mathbf{x}}$ & 1 & $e^{\pi i \frac{15+2 r}{16}}$ & $e^{i \pi / 2}$ & $e^{\pi i \frac{3+2 r}{16}}$ & $e^{-i \pi / 2}$ & $e^{\pi i \frac{15+2 r}{16}}$ & -1 \\
& & & $r$ odd & & &
\end{tabular}

Table 4.3: The exchange phase $\theta_{\mathbf{x}}=e^{2 \pi i h_{\mathbf{x}}}$ and quantum dimensions of anyons $\mathbf{x}$ in a $(2+1) \mathrm{D} S O(3)_{3} \otimes_{b} S O(r)_{1}$ topological phase.

For odd $N=9+2 r$, the $\mathcal{G}_{N}$ WZW CFT at the interface corresponds the TR symmetric gapped surface that carries a topological order given by the relative tensor 
product

$$
G_{N}=S O(3)_{3} \otimes_{b} S O(r)_{1}
$$

where the fermion pair $b=\psi_{S O(3)_{3}} \times \psi_{S O(r)_{1}}$ is condensed. The concept of anyon condensation[9] will be demonstrated more explicitly later in section 4.3.2. The topological state carries seven types of anyons and are summarized in table 4.3. For instance, the anyon structure matches the primary field content of the $s o(3)_{3}$ WZW theory (see table 4.1) when $r=0$. The quasiparticle fusion rules of $G_{N}$ are similar to the $s o(3)_{3}$ ones in (4.89)

$$
\begin{gathered}
f \times f=1, \quad f \times \gamma_{ \pm}=\gamma_{\mp}, \quad f \times \alpha_{ \pm}=\alpha_{\mp}, \quad f \times \beta=\beta \\
\gamma_{ \pm} \times \gamma_{ \pm}=1+\gamma_{+}+\gamma_{-}, \quad \alpha_{ \pm} \times \beta=\gamma_{+}+\gamma_{-} \\
\beta \times \beta=1+\gamma_{+}+\gamma_{-}+f, \quad \beta \times \gamma_{ \pm}=\alpha_{+}+\alpha_{-}+\beta
\end{gathered}
$$

except the following modifications that dependent on $r=(N-9) / 2$.

$$
\begin{gathered}
\alpha_{ \pm} \times \alpha_{ \pm}= \begin{cases}1+\gamma_{+}, & \text {for } r \equiv 0 \bmod 4 \\
f+\gamma_{+}, & \text {for } r \equiv 1 \bmod 4 \\
f+\gamma_{-}, & \text {for } r \equiv 2 \bmod 4 \\
1+\gamma_{-}, & \text {for } r \equiv 3 \bmod 4\end{cases} \\
\alpha_{ \pm} \times \gamma_{ \pm}= \begin{cases}\alpha_{+}+\beta, & \text { for } r \text { even } \\
\alpha_{-}+\beta, & \text { for } r \text { odd }\end{cases}
\end{gathered}
$$

This quasiparticle spin and fusion structure will be shown later in section 4.3.2. The braiding $S$-matrices of the $G_{N}$ states are summarized in appendix E. 
The $G_{N}$ sequence extends the sixteenfold periodic anyon structure[60, 59, 118] $S O(r+16)_{1} \cong S O(r)_{1}$ to a periodic class of thirty two topological states

$$
G_{N+32} \cong G_{N}
$$

This seemingly contradicts the sixteenfold prediction of topologically ordered surface states from Ref.[32, 74, 130, 111, 56, 95, 141]. This is due to the non-local nature of the "antiferromagnetic" time reversal symmetry in the coupled Majorana wire model. On the other hand, in general there are multiple possible gapping potentials that leads to distinct topological order. For instance, we will show in a subsequent section that for $N=16$, there is an extended $E_{8}$ symmetry or an alternative conformal embedding that would allow a different set of gapping terms but would forbid all electronic quasiparticle excitations.

The thirty two topological states here follow a $\mathbb{Z}_{32}$ tensor product algebraic structure

$$
G_{N_{1}} \otimes_{b} G_{N_{2}} \cong G_{N_{1}+N_{2}}
$$

where certain maximal set of mutually local bosons from $G_{N_{1}}$ and $G_{N_{2}}$ are pair condensed in the relative tensor product. We will discuss this in more detail below.

\subsubsection{The 32-fold tensor product structure}

We first explain the relative tensor product that defines the $G_{N}$ topological state in eq.(4.126). We begin with the tensor product state $S O(3)_{3} \otimes S O(r)_{1}$ which consists of decoupled $S O(3)_{3}=S U(2)_{6}$ and $S O(r)_{1}$ topological states. The primary fields of the $s u(2)_{6}$ WZW CFT are labeled by seven half-integral "spins"

$s=\mathbf{0}, \mathbf{1} / \mathbf{2}, \mathbf{1}, \mathbf{3} / \mathbf{2}, \mathbf{2}, \mathbf{5} / \mathbf{2}, \mathbf{3}$ and are summarized in table 4.1 and eq.(4.89). These correspond to the anyon structure of the $(2+1) \mathrm{D} S O(3)_{3}$ topological state. The 
topological order of $S O(r)_{1}$ is well-known[60] and was summarized earlier in this section. For instance, "spin" 3 corresponds to the BdG fermion quasiparticle $f$, and the half-integral "spins" $\mathbf{1 / 2}, \mathbf{3} / \mathbf{2}$ and $\mathbf{5 / 2}$ are $\pi$-fluxes that give a -1 monodromy phase of an orbiting fermion.

In the coupled Majorana wire model where there are $N=9+2 r$ Majorana channels per wire, the gapping term explicitly seperates the first 9 and final $2 r$ channels and the current backscattering potential does not mix these two sectors. This model would therefore give a decouple $S O(3)_{3} \otimes S O(r)_{1}$ topological state. However, there could be additional local time reversal symmetric terms, such as intrawire forward scattering $i \psi_{a}^{R} \psi_{b}^{R}$ and $i \psi_{a}^{L} \psi_{b}^{L}$, that mixes the two sectors and condenses the fermion pair $b=$ $f_{S O(3)_{3}} \otimes \psi_{S O(r)_{1}}$. In fact, fermion pair condensation is natural in a superconducting medium where the ground state consists of Cooper pairs. The condensation of the bosonic anyon $b$ results in the confinement of certain quasiparticles that have nontrivially monodromy around it.[9] These includes all the $\pi$ fluxes $\mathbf{1 / 2}, \mathbf{3 / 2}$ and $\mathbf{5 / 2}$ in the $S O(3)_{3}$ sector, $s_{ \pm}$(or $\left.\sigma\right)$ in $S O(r)_{1}$ for $r$ even (resp. odd), as well as the tensor product $\mathbf{1} / \mathbf{2} \otimes \psi, \mathbf{3} / \mathbf{2} \otimes \psi, \mathbf{5} / \mathbf{2} \otimes \psi, \mathbf{1} \otimes s_{ \pm}, \mathbf{2} \otimes s_{ \pm}$and $\mathbf{3} \otimes s_{ \pm}($or $\mathbf{1} \otimes \sigma$, $\mathbf{2} \otimes \sigma$ and $\mathbf{3} \otimes \sigma)$. The remaining anyons are local with respect to the boson $b$ and survive the condensation, but certain pairs are identified if they differ only by the boson condensate, $\mathbf{a} \times b \equiv \mathbf{a}$. This includes $\mathbf{3} \equiv \psi, \mathbf{1} \otimes \psi \equiv \mathbf{2}, \mathbf{2} \otimes \psi \equiv \mathbf{1}$, $\mathbf{1} / \mathbf{2} \otimes s_{ \pm} \equiv \mathbf{5} / \mathbf{2} \otimes s_{\mp}$ and $\mathbf{3} / \mathbf{2} \otimes s_{+} \equiv \mathbf{3} / \mathbf{2} \otimes s_{-}$for even $r$, or $\mathbf{1} / \mathbf{2} \otimes \sigma \equiv \mathbf{5} / \mathbf{2} \otimes \sigma$ for $r$ odd. Special care has to be taken for the tensor product $\mathbf{3} / \mathbf{2} \otimes \sigma$ when $r$ is odd. After condensation, the fusion rule of a pair of $\mathbf{3} / \mathbf{2} \otimes \sigma$ becomes

$$
\begin{aligned}
(\mathbf{3} / \mathbf{2} \otimes \sigma) \times(\mathbf{3} / \mathbf{2} \otimes \sigma) & =(\mathbf{0}+\mathbf{1}+\mathbf{2}+\mathbf{3}) \otimes(1+\psi) \\
& \equiv \mathbf{0}+\mathbf{0}+\mathbf{1}+\mathbf{1}+\mathbf{2}+\mathbf{2}+\mathbf{3}+\mathbf{3}
\end{aligned}
$$


which has two vacuum fusion channels and indicates that $\mathbf{3} / \mathbf{2} \otimes \sigma$ cannot be a simple object. This leads to the decomposition

$$
\mathbf{3} / \mathbf{2} \otimes \sigma \equiv \alpha_{+}+\alpha_{-}
$$

where $\alpha_{ \pm}$are simple anyons with identical exchange statistics but opposite fermion parity $\alpha_{ \pm} \times f=\alpha_{\mp}$ and obey the fusion rules (4.128).

\begin{tabular}{l|lllllll} 
& 1 & $\alpha_{+}$ & $\gamma_{+}$ & $\beta$ & $\gamma_{-}$ & $\alpha_{-}$ & $f$ \\
\hline$r$ even & $\mathbf{0}$ & $\mathbf{1 / 2} \otimes s_{+}$ & $\mathbf{1}$ & $\mathbf{3} / \mathbf{2} \otimes s_{ \pm}$ & $\mathbf{2}$ & $\mathbf{5 / 2} \otimes s_{+}$ & $\mathbf{3}$ \\
$r$ odd & $\mathbf{0}$ & $(\mathbf{3} / \mathbf{2} \otimes \sigma)_{+}$ & $\mathbf{1}$ & $\mathbf{1} / \mathbf{2} \otimes \sigma$ & $\mathbf{2}$ & $(\mathbf{3} / \mathbf{2} \otimes \sigma)_{-}$ & $\mathbf{3}$
\end{tabular}

Table 4.4: Identification of the seven anyon types in table 4.3 as tensor products.

We summarize the identification of the seven anyon types in $G_{N}=S O(3)_{3} \nabla_{b}$ $S O(r)_{1}$ as tensor products in table 4.4. This explains the exchange statistics and quantum dimensions of the quasiparticles in table 4.3

$$
\theta_{\mathbf{a} \otimes \mathbf{b}}=\theta_{\mathbf{a}} \theta_{\mathbf{b}}, \quad d_{\mathbf{a} \otimes \mathbf{b}}=d_{\mathbf{a}} d_{\mathbf{b}}
$$

with the exception of the non-simple object $\mathbf{3} / \mathbf{2} \otimes \sigma$ in (4.132) where each component $\alpha_{ \pm}$carries half of its dimension. The fusion rules in (4.127) and (4.128) are explained by the tensor product

$$
\left(\mathbf{a}_{1} \otimes \mathbf{b}_{1}\right) \times\left(\mathbf{a}_{2} \otimes \mathbf{b}_{2}\right)=\left(\mathbf{a}_{1} \times \mathbf{a}_{2}\right) \otimes\left(\mathbf{b}_{1} \times \mathbf{b}_{2}\right)
$$

except in the odd $r$ cases where again the non-simple object $\mathbf{3} / \mathbf{2} \otimes \sigma=\alpha_{+}+\alpha_{-}$ requires special attention.

The fusion rules (4.128) of $\alpha_{ \pm}$in the odd $r$ cases are fixed by modular invariance. The braiding $S$-matrix (4.118) is determined by fusion rules and quasiparticle exchange statistics. On the other hand fusion rules can also be determined by the $S$ - 
matrix using the Verlinde formula (4.87).[123] Moreover one can define the $T$-matrix according to the quasiparticle exchange statistics

$$
T_{\mathrm{ab}}=\delta_{\mathrm{ab}} \theta_{\mathbf{a}}
$$

which corresponds to the modular $T$-transformation in the CFT along the boundary. As a consequence they satisfies the $S L(2 ; \mathbb{Z})$ algebraic relation[60]

$$
\left(\mathcal{S} T^{\dagger}\right)^{3}=e^{-2 \pi i c_{-} / 8} \mathcal{S}^{2}
$$

where $c_{-}=c_{R}-c_{L}$ is the chiral central charge of the corresponding CFT along the boundary

$$
c_{-}\left(G_{N}\right)=c_{-}\left(s o(3)_{3}\right)+c_{-}\left(s o(r)_{1}\right)=\frac{9}{4}+\frac{r}{2}=\frac{N}{4} .
$$

These put a very restrictive constraint on the allowed topological field theory and fix the fusion rules (4.128) for $\alpha_{ \pm}$when $r$ is odd. The braiding $S$ matrices can be found in appendix E.

The relative tensor product structure of the sixteenfold $S O(r)_{1}$ sequence itself can also be understood using anyon condensation

$$
S O\left(r_{1}\right)_{1} \otimes_{b} S O\left(r_{2}\right)_{1} \cong S O\left(r_{1}+r_{2}\right)_{1}
$$

where the fermion pair $\psi_{1} \otimes \psi_{2}$ is condensed. This can be verified by a similar condensation procedure as the one presented above. For instance, if $r_{1}$ and $r_{2}$ are both odd, the tensor product $\sigma_{1} \otimes \sigma_{2}$ will become non-simple after condensation and decompose into a pair of abelian $\pi$-fluxes, $s_{+}+s_{-}$, with identical exchange 
statistics but opposite fermion parities $s_{ \pm} \times \psi=s_{\mp}$ and are related by an anyonic symmetry[59, 118].

Next we move on to explaining the general relative tensor product structure (4.130) of the 32 -fold $G_{N}$ states. Eq.(4.138) describes the cases when both $N_{1}$ and $N_{2}$ are even, i.e. $G_{2 r_{1}} \bigotimes_{b} G_{2 r_{2}} \cong G_{2 r_{1}+2 r_{2}}$. A similar anyon condensation procedure that defined the relative tensor product $S O(3)_{3} \otimes_{b} S O(r)_{1}$ above would prove that

$$
G_{N} \bigotimes_{b} S O(r)_{1} \cong G_{N+2 r}
$$

for $N$ odd, where the fermion pair $b=f_{G_{N}} \otimes \psi_{S O(r)_{1}}$ is condensed.

When both $N_{1}=9+2 r_{1}$ and $N_{2}=9+2 r_{2}$ are odd, each of the two $G_{N_{i}}=$ $S O(3)_{3} \otimes_{b} S O\left(r_{i}\right)_{1}$ theories contains seven anyon types $1, \alpha_{ \pm}^{i}, \gamma_{ \pm}^{i}, \beta^{i}, f^{i}$. The tensor product state $G_{N_{1}} \otimes G_{N_{2}}$ contains three non-trivial bosons

$$
b=\left\{b_{0}, b_{+}, b_{-}\right\}=\left\{f^{1} \otimes f^{2}, \gamma_{+}^{1} \otimes \gamma_{-}^{2}, \gamma_{-}^{1} \otimes \gamma_{+}^{2}\right\}
$$

as $\gamma_{ \pm}$have conjugate exchange phases $\theta_{\gamma_{ \pm}}= \pm i$. Moreover, these bosons are mutually local. Firstly, $b_{0}$ have trivial monodromy around $b_{ \pm}$as $\gamma_{ \pm}$are local with respect to the fermion $f$. Secondly, as there are bosonic fusion channels $b_{ \pm} \times b_{ \pm}=1+b_{+}+b_{-}+\ldots$ and $b_{ \pm} \times b_{\mp}=b_{0}+b_{+}+b_{-}+\ldots, b_{ \pm}$are local among themselves because their mutual monodromy phases are trivial. We first condensed the Abelian fermion pair $b_{0}=f^{1} \otimes f^{2}$. The resulting theory contains the following set of (non-confined) anyon types

$$
G_{N_{1}} \otimes_{b_{0}} G_{N_{2}}=\left\langle\begin{array}{c}
1, f, \gamma_{ \pm}^{1}, \gamma_{ \pm}^{2}, \gamma_{+}^{1} \gamma_{+}^{2}, \gamma_{+}^{1} \gamma_{-}^{2}, \\
\alpha_{+}^{1} \alpha_{+}^{2}, \alpha_{+}^{1} \alpha_{-}^{2}, \alpha_{+}^{1} \beta^{2}, \beta^{1} \alpha_{+}^{2}, \beta^{1} \beta^{2}
\end{array}\right\rangle
$$

where some anyon types are identified by the $b_{0}$ condensate, such as $f \equiv f^{1} \equiv f^{2}$ and $\gamma_{-}^{1} \gamma_{-}^{2}=\gamma_{+}^{1} \gamma_{+}^{2} \times b_{0}$, and are therefore not listed. Next we condense the non- 
Abelian boson $b_{+}=\gamma_{+}^{1} \gamma_{-}^{2}$, which is already equated with $b_{-}=b_{+} \times b_{0}$. The general condensation procedure of a non-Abelian boson was proposed by Bais and Slingerland in Ref.[9]. In the present case, it begins with the fusion theory $\mathcal{F}$ of $G_{N_{1}} \otimes_{b_{0}} G_{N_{2}}$ that only encodes the associative fusion content but neglects the braiding structure of the anyons. As the boson $b_{+}$is condensed, it decomposes as $b_{+}=\gamma_{+}^{1} \gamma_{-}^{2}=1+\ldots$, which now contains the vacuum channel 1 . This reduces the fusion theory $\mathcal{F}$ into a new fusion theory $\mathcal{F}^{\prime}$, where the certain anyons in (4.141) become non-simple objects and decompose into simpler components while others are identified by the boson condensate. This new fusion category $\mathcal{F}^{\prime}$ contains the non-confined anyons in the resulting state as well as confined non-point-like objects.

We start with the first line of anyons in (4.141), which are all local with respect to the fermion $f$. The semion $\gamma_{+}^{1}$ is self-conjugate as $\gamma_{+}^{1} \times \gamma_{+}^{1}=1+\gamma_{+}^{1}+\gamma_{-}^{1}$. However $\gamma_{-}^{2}$ is now also an antiparticle of $\gamma_{+}^{1}$ since $\gamma_{+}^{1} \times \gamma_{-}^{2}=b_{+}=1+\ldots$ also contains the vacuum channel. The uniqueness of antipartner guarantees the identifications

$$
\gamma_{+} \equiv \gamma_{+}^{1} \equiv \gamma_{-}^{2}, \quad \gamma_{-} \equiv \gamma_{-}^{1} \equiv \gamma_{+}^{2}
$$

which obey the usual fusion rules $\gamma_{ \pm} \times \gamma_{ \pm}=1+\gamma_{+}+\gamma_{-}$and $f \times \gamma_{ \pm}=\gamma_{\mp}$. This in turn determines the decomposition of the non-Abelian boson

$$
b_{+}=\gamma_{+}^{1} \gamma_{-}^{2} \equiv \gamma_{+} \times \gamma_{+}=1+\gamma_{+}+\gamma_{-}
$$

which is consistent with the boson quantum dimension $d_{b_{+}}=d_{\gamma}^{2}=1+2 d_{\gamma}$. Moreover the non-Abelian fermion also decomposes

$$
\gamma_{+}^{1} \gamma_{+}^{2} \equiv \gamma_{+} \times \gamma_{-}=f+\gamma_{+}+\gamma_{-} .
$$


Next we move on to the second line of anyons in (4.141), which are $\pi$ fluxes with respect to the fermion $f$. From the original fusion rules (4.127), (4.128) and the identification (4.142), (4.143) and (4.144), the $\pi$ fluxes satisfy the fusion rules

$$
\begin{aligned}
& \left(\alpha_{+}^{1} \alpha_{+}^{2}\right) \times\left(\alpha_{+}^{1} \alpha_{+}^{2}\right) \\
& = \begin{cases}1+f+2 \gamma_{+}+2 \gamma_{-}, & \text {for } r_{1}+r_{2} \text { even } \\
1+1+\gamma_{+}+\gamma_{-}+2 \gamma_{ \pm}, & \text {for } r_{1}+r_{2} \equiv 3 \bmod 4 \\
f+f+\gamma_{+}+3 \gamma_{-}, & \text {for } r_{1}+r_{2} \equiv 1 \bmod 4\end{cases}
\end{aligned}
$$

$$
\begin{gathered}
\left(\alpha^{1} \beta^{2}\right) \times\left(\alpha^{1} \beta^{2}\right)=1+1+f+f+4 \gamma_{+}+4 \gamma_{-} \\
\left(\beta^{1} \beta^{2}\right) \times\left(\beta^{1} \beta^{2}\right)=4\left(1+f+2 \gamma_{+}+2 \gamma_{-}\right) \\
\left(\alpha_{+}^{1} \alpha_{+}^{2}\right) \times\left(\alpha_{+}^{1} \beta^{2}\right)=1+f+3 \gamma_{+}+3 \gamma_{-} \\
\left(\alpha_{+}^{1} \alpha_{+}^{2}\right) \times\left(\beta^{1} \beta^{2}\right)=1+1+f+f+4 \gamma_{+}+4 \gamma_{-}
\end{gathered}
$$

for $N_{1}=9+2 r_{1}$ and $N_{2}=9+2 r_{2}$.

These show $\alpha^{1} \beta^{2}$ and $\beta^{1} \beta^{2}$ must be non-simple because their corresponding fusion rules contain multiple vacuum channels. The decomposition of $\beta^{1} \beta^{2}$ is simplest and applies to all $r_{1}, r_{2}$

$$
\beta^{1} \beta^{2}=\alpha_{+}^{1} \alpha_{+}^{2}+\alpha_{+}^{1} \alpha_{-}^{2}
$$

where $\alpha_{+}^{1} \alpha_{-}^{2}=\alpha_{+}^{1} \alpha_{+}^{2} \times f$. For instance, it is straightforward to check that this decomposition is consistent with the fusion rules. $\alpha_{+}^{1} \beta^{2}$ and $\alpha_{-}^{1} \beta^{2}$ are clearly identified as they differ only by the Abelian boson $b_{0}=f^{1} f^{2}$. We therefore will simply denote them as $\alpha^{1} \beta^{2}$. Moreover, one can show that $\alpha^{1} \beta^{2}$ and $\beta^{1} \alpha^{2}$ are also identified after the condensation of the non-Abelian boson $\gamma_{+}^{1} \gamma_{-}^{2}=1+\gamma_{+}+\gamma_{-}$in (4.143). This can 
be verify by equating the fusion equations $\left(\alpha^{1} \beta^{2}\right) \times\left(\gamma_{+}^{1} \gamma_{-}^{2}\right)=\left(\alpha^{1} \beta^{2}\right) \times\left(1+\gamma_{+}+\gamma_{-}\right)$. The decomposition of $\alpha^{1} \beta^{2} \equiv \beta^{1} \alpha^{2}$ depends on the parity of $r_{1}+r_{2}$.

When $r_{1}+r_{2}$ is even, the pair fusion rule for $\alpha_{+}^{1} \alpha_{+}^{2}$ allows it to be simple since there is a unique vacuum channel. Moreover as the pair fusion rule is unaltered by the addition of a fermion $f$, it is identical to $\left(\alpha_{+}^{1} \alpha_{+}^{2}\right) \times\left(\alpha_{+}^{1} \alpha_{-}^{2}\right)$. This shows $\alpha_{ \pm}^{1} \alpha_{-}^{2}$ conjugates and therefore identifies with $\alpha_{ \pm}^{1} \alpha_{+}^{2}$, which is self-conjugate.

$$
\alpha^{1} \alpha^{2} \equiv \alpha_{ \pm}^{1} \alpha_{ \pm}^{2} \equiv \alpha_{ \pm}^{1} \alpha_{\mp}^{2} .
$$

In this case, $\alpha^{1} \beta^{2}$ is decomposed into

$$
\alpha^{1} \beta^{2}=\sigma+\alpha^{1} \alpha^{2}
$$

where we introduce the Ising anyon $\sigma$ that obey

$$
\begin{gathered}
\sigma \times \sigma=1+f, \quad \sigma \times f=\sigma \\
\sigma \times \alpha^{1} \alpha^{2}=\gamma_{+}+\gamma_{-}, \quad \sigma \times \gamma_{ \pm}=\alpha^{1} \alpha^{2} .
\end{gathered}
$$

The decomposition (4.152) is consistent with the fusion rules (4.148) and (4.146). The reduced fusion category after condensing the boson (4.143) is therefore generated by the following simple objects

$$
\mathcal{F}_{\text {even }}^{\prime}=\left\langle 1, f, \sigma, \gamma_{ \pm}, \alpha^{1} \alpha^{2}\right\rangle
$$

when $r_{1}+r_{2}$ is even. It has the fusion rules (4.153) together with $\gamma_{ \pm} \times \alpha^{1} \alpha^{2}=$ $\sigma+2 \alpha^{1} \alpha^{2}$.

When $r_{1}+r_{2}$ is odd, we need to further separate into two cases. When $r_{1}+r_{2} \equiv 3$ mod 4, the fusion rule of a pair of $\alpha_{+}^{1} \alpha_{+}^{2}$ in (4.145) forbids it to be simple. It 
decomposes into

$$
\alpha_{+}^{1} \alpha_{+}^{2}=s_{+}+\gamma_{+} \quad \text { or } \quad s_{+}+\gamma_{-}
$$

where $s_{ \pm}$are Abelian anyons that satisfy the fusion rules

$$
s_{ \pm} \times s_{ \pm}=1, \quad s_{ \pm} \times f=s_{\mp}, \quad s_{+} \times \gamma_{ \pm}=\gamma_{ \pm}
$$

and the fermion parity $\gamma_{ \pm}$in $(4.155)$ depends on $\left(r_{1}, r_{2}\right) \equiv(0,3)$ or $(1,2) \bmod 4$ but is unimportant for the current discussion. The decomposition (4.155) is consistent with the fusion rule (4.145). In this case, the fusion rules $\left(\alpha_{+}^{1} \alpha_{+}^{2}\right) \times\left(\alpha^{1} \beta^{2}\right)$ in (4.148) requires a different decomposition of $\alpha^{1} \beta^{2}$ than (4.152).

$$
\alpha^{1} \beta^{2}=\gamma_{+}+\gamma_{-} .
$$

The reduced fusion category after condensing the boson (4.143) is therefore generated by the following simple objects

$$
\mathcal{F}_{3}^{\prime}=\left\langle 1, f, s_{ \pm}, \gamma_{ \pm}\right\rangle
$$

when $r_{1}+r_{2} \equiv 3 \bmod 4$.

When $r_{1}+r_{2} \equiv 1 \bmod 4$, the fusion rule (4.145) again forbids $\alpha_{+}^{1} \alpha_{+}^{2}$ to be simple. Moreover as the vacuum channel is absent, it is no longer self-conjugate but instead is conjugate with $\alpha_{+}^{1} \alpha_{-}^{2}$ since it has opposite fermion parity and $\left(\alpha_{+}^{1} \alpha_{+}^{2}\right) \times\left(\alpha_{+}^{1} \alpha_{-}^{2}\right)=$ $1+1+3 \gamma_{+}+\gamma_{-}$. We decompose

$$
\alpha_{+}^{1} \alpha_{+}^{2}=s_{+}+g_{+}
$$


where $s_{ \pm}$are Abelian anyons and $g_{ \pm}$are non-Abelian objects that satisfy

$$
s_{ \pm} \times s_{ \pm}=f, \quad s_{ \pm} \times f=s_{\mp}, \quad g_{ \pm}=\gamma_{+} \times s_{ \pm} .
$$

The decomposition of $\alpha^{1} \beta^{2}$ also needs to be modified

$$
\alpha^{1} \beta^{2}=g_{+}+g_{-} .
$$

One can check that these decompositions are consistent with the original fusion rules. The reduced fusion category after condensing the boson (4.143) is therefore generated by the following simple objects

$$
\mathcal{F}_{1}^{\prime}=\left\langle 1, f, s_{ \pm}, \gamma_{ \pm}, g_{ \pm}\right\rangle
$$

when $r_{1}+r_{2} \equiv 1 \bmod 4$.

Not all objects in the reduced fusion theories $\mathcal{F}_{\text {even }}^{\prime}, \mathcal{F}_{1}^{\prime}$ and $\mathcal{F}_{3}^{\prime}$ in (4.154), (4.162) and (4.158) are non-confined anyons in the new topological states. Some may be non-local with respect to the boson $b_{+}(4.143)$ and are therefore not point-like objects when $b_{+}$is condensed. They are equipped with a physical string or branch cut that extends. The anyon theory, which encodes both fusion and braiding information, after condensation excludes these confined extended objects. To determine which objects in the reduced fusion categories $\mathcal{F}^{\prime}$ are non-confined anyons, we look at the possible monodromy around the condensed boson $b_{+}$. Suppose $\mathbf{a}_{1} \otimes \mathbf{a}_{2}$ and $\mathbf{b}_{1} \otimes \mathbf{b}_{2}$ are anyons in the tensor product state $G_{N_{1}} \bigotimes_{b_{0}} G_{N_{2}}$ (4.141) that are related by the fusion rule 
$b_{+} \times\left(\mathbf{a}_{1} \otimes \mathbf{a}_{2}\right)=\mathbf{b}_{1} \otimes \mathbf{b}_{2}+\ldots$, the monodromy under this fixed fusion channel is $[9]$

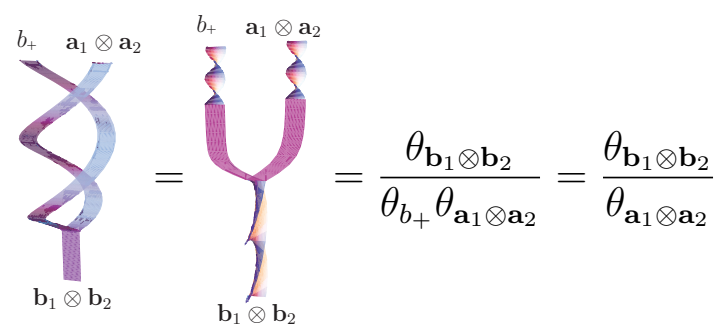

as $b_{+}$is a boson with $\theta_{b_{+}}=1$. In other words trivial monodromy simply reqires the invariance of exchange statistics upon an addition of the boson.

Given any simple object $\mathbf{x}$ in the reduced fusion category $\mathcal{F}^{\prime}$ in $(4.154)$, (4.162) or (4.158), it may be lifted to multiple anyons in the tensor product state $G_{N_{1}} \otimes_{b_{0}} G_{N_{2}}$ in (4.141) in the sense that it belongs in distinct decompositions $\mathbf{a}_{1} \otimes \mathbf{a}_{2}=\mathbf{x}+\ldots$ and $\mathbf{b}_{1} \otimes \mathbf{b}_{2}=\mathbf{x}+\ldots$ For instance, $\gamma_{ \pm}$are components of the boson $\gamma_{+}^{1} \gamma_{-}^{2}=1+\gamma_{+}+\gamma_{-}$ as well as the fermion $\gamma_{+}^{1} \gamma_{+}^{2}=f+\gamma_{+}+\gamma_{-}$(see (4.143) and (4.144)). If $\mathbf{x}$ is an object not confined by the boson condensation, then its exchange statistics should be independent from the choices of lift

$$
\theta_{\mathbf{x}}=\theta_{\mathbf{a}_{1} \otimes \mathbf{a}_{2}}=\theta_{\mathbf{b}_{1} \otimes \mathbf{b}_{2}}
$$

since the monodromy (4.163) should be trivial. Otherwise, the object $\mathbf{x}$ has to be non-point-like and extended as it does not have well defined statistics. For example since $\gamma_{ \pm}$belongs to the decomposition of a non-Abelian boson and fermion, they have to be confined objects after condensation.

The relative tensor product $G_{N_{1}} \bigotimes_{b} G_{N_{2}}$ with the condensation of the set of bosons $b(4.140)$ contains non-confined anyons in the reduced fusion categories $\mathcal{F}_{\text {even }}^{\prime}, \mathcal{F}_{1}^{\prime}$ and $\mathcal{F}_{3}^{\prime}$ in (4.154), (4.162) and (4.158). For example when $r_{1}+r_{2}$ is even, the simple object $\alpha^{1} \alpha^{2}$ in (4.154) is confined and is not an anyon because it can be lifted into $\alpha^{1} \beta^{2}$ and $\beta^{1} \beta^{2}$, which have distinct statistics, in (4.152) and (4.150). When $r_{1}+r_{2} \equiv 1 \bmod 4$, 
the simple objects $g_{ \pm}$are also confined because they belong in $\alpha^{1} \beta^{2}$ and $\alpha_{+}^{1} \alpha_{ \pm}^{2}$, which have different spins, in (4.161) and (4.159). This shows $G_{N_{1}} \otimes_{b} G_{N_{2}}$ is generated by the non-confined anyons

$$
G_{N_{1}} \otimes_{b} G_{N_{2}}=\left\{\begin{array}{cc}
\langle 1, f, \sigma\rangle, & \text { for } r_{1}+r_{2} \text { even } \\
\left\langle 1, f, s_{ \pm}\right\rangle, & \text {for } r_{1}+r_{2} \text { odd }
\end{array}\right.
$$

The exchange statistics of $\sigma$ and $s_{ \pm}$are determined by that of their lifts. For instance,

$$
\theta_{\sigma}=\theta_{\alpha^{1} \beta^{2}}=\theta_{\alpha} \theta_{\beta}=e^{\pi i \frac{9+r_{1}+r_{2}}{8}}=e^{\pi i\left(N_{1}+N_{2}\right) / 16}
$$

using table 4.3 when $r_{1}+r_{2}$ is even. This shows

$$
G_{N_{1}} \otimes_{b} G_{N_{2}}=S O\left(\frac{N_{1}+N_{2}}{2}\right)_{1}
$$

when both $N_{1}$ and $N_{2}$ are odd and concludes the 32 -fold tensor product algebraic structure of the $G_{N^{-}}$series.

\subsection{Other possibilities}

In the previous sections, we proposed time reversal symmetric interactions that gap the coupled Majorana wire model and lead to a $G_{N}$ topological order (see eq.(4.123) and (4.126)). The interwire current-current backscattering interactions depend on a particular fractionalization, so(N) $)_{1} \supseteq \mathcal{G}_{N} \times \mathcal{G}_{N}$, of the $N$ Majorana channels per wire. However, in special cases, we have already seen that alternative decompositions exist and correspond to different gapping interactions and topological orders. For example, at the beginning of section 4.2, we showed when there are even Majorana channels per wire, the model could simply be gapped by a singlebody backscattering potential (see (4.30)) and have trivial topological order. This 
is consistent with the $\mathbb{Z}_{2}$ classification of gapless Majorana modes protected by the "antiferromagnetic" time reversal symmetry (4.1). Another example was given in section 4.2.1.2 for the special case when there are $N=4$ Majorana channels per wire where the decomposition needs to be changed into $s o(4)_{1} \supseteq s u(2)_{1} \times s u(2)_{1}$. The resulting gapped state carries the $S U(2)_{1}$ semion topological order instead of $G_{4}=S O(2)_{1}$

Moreover the sixteenfold classification of topological superconductors (TSC) with the presence of interaction[32, 74, 130, 111, 56, 95, 141] suggests the 32 -fold $G_{N}$-series could have redundancies. On the other hand, the $\mathbb{Z}_{16}$ classification of TSC is based on the canonical local time reversal symmetry, which is fundamentally different from the non-local "antiferromagnetic" time reversal considered in this manuscript. The $\mathbb{Z}_{32}$ structure of surface topological order could be an artifact of such unconventional time reversal symmetry. Nonetheless, here in section 4.4.1 and 4.4.2, we discuss altenative gapping interactions when $N=16$ that removes all electronic quasiparticles.

\subsubsection{Consequence of the emergent $E_{8}$ when $N=16$}

We design alternative interwire backscattering terms in the coupled wire model (4.3) with $N=16$ Majorana channels per wire. They open a time reversal symmetric energy gap among 16 surface Majorana cones with the same chirality. In general, these terms can also apply when the number of chiral Majorana channel per wire is larger than 16 by acting on a subset of channels. We begin with the bosonized description presented previously in section 4.1.2, where each wire consists of an 8-component chiral $U(1)$ boson $\widetilde{\phi}=\left(\tilde{\phi}^{1}, \ldots, \tilde{\phi}^{8}\right)$ that bosonizes the complex fermions $c_{j}=\left(\psi_{2 j-1}+\right.$ $\left.i \psi_{2 j}\right) / \sqrt{2}=\exp \left(i \tilde{\phi}^{j}\right)$. This theory is special because it carries non-trivial bosonic primary fields, which can condense. For example the two spinor representations $s_{ \pm}$ correspond to bosonic primary fields of so(16) $)_{1}$ with conformal dimension $h_{s_{ \pm}}=1$ (see eq.(4.17)). In particular we will focus on the even sector $s_{+}$. It consists of vertex 
operators

$$
V_{s_{+}}^{\varepsilon}=e^{i \varepsilon \cdot \tilde{\phi} / 2}, \quad \varepsilon=\left(\varepsilon_{1}, \ldots, \varepsilon_{8}\right)
$$

(see eq.(B.24)) for $\varepsilon_{j}= \pm 1$ with $\varepsilon_{1} \ldots \varepsilon_{8}=+1$. The $128=2^{7}$ number of combinations naturally matches with the dimension of the even spinor representation of $s o(16)$ (see appendix A). These $V_{s_{+}}^{\varepsilon}$ are related to each other through the OPE with the raising and lowering operators $E^{\alpha}=e^{i \alpha \cdot \widetilde{\phi}}=e^{i\left( \pm \tilde{\phi}^{i} \pm \tilde{\phi}^{j}\right)}$ of $s o(16)_{1}$ (see (B.8) in appendix B). The 128 lattice vectors $\varepsilon / 2$ extend the 112 roots $\boldsymbol{\alpha}$ of $s o(16)$ to the root lattice of the exceptional simple Lie algebra $E_{8}$ with size 240.[26] The unit dimensional vertex operators $V_{s_{+}}^{\varepsilon}$ themselves can be regarded as raising and lowering operators that enlarge the $s o(16)_{1}$ current algebra to $E_{8}$ at level 1 . This extends the current algebra of each wire

$$
s o(16)_{1} \subseteq\left(E_{8}\right)_{1}
$$

and is intimately related to the fact that the surface state can be gapped out without leaving electronic quasiparticles which are non-local with respect to the boson $s_{+}$.

The gapping strategy is to condense primary fields in the bosonic sector $s_{+}$between adjacent wires. This is facilitated by interwire backscattering interactions that bipartite the emergent $E_{8}$ symmetry.

$$
E_{8} \supseteq \widetilde{s o(8)_{1}^{+}} \times \widetilde{s o(8)_{1}^{-}}
$$

However, these $\widetilde{s o(8)_{1}}$ subalgebras are distinct from the ones in the decomposition $s o(16)_{1} \supseteq s o(8)_{1} \times s o(8)_{1}$. In particular, we will see that they do not support electronic primary fields $c_{j}=e^{i \tilde{\phi}^{j}}$. Out of $128 \varepsilon$ lattice vectors in (4.168), there is a (non-unique) 
maximal set of 8 orthonormal vectors $\boldsymbol{\varepsilon}_{(1)}, \ldots, \boldsymbol{\varepsilon}_{(8)}$

$$
\frac{1}{2} \varepsilon_{(m)} \cdot \frac{1}{2} \varepsilon_{(n)}=2 \delta_{m n}
$$

We choose the set containing the highest weight vector $\varepsilon_{(1)}=(1,1,1,1,1,1,1,1)$ :

$$
\left(\begin{array}{ccc}
\mid & & \mid \\
& & \\
\boldsymbol{\varepsilon}_{(1)} & \ldots & \boldsymbol{\varepsilon}_{(8)} \\
\mid & & \mid
\end{array}\right)=\left(\begin{array}{cccccccc}
1 & 1 & 1 & 1 & 1 & 1 & 1 & 1 \\
1 & 1 & 1 & 1 & -1 & -1 & -1 & -1 \\
1 & 1 & -1 & -1 & 1 & 1 & -1 & -1 \\
1 & 1 & -1 & -1 & -1 & -1 & 1 & 1 \\
1 & -1 & 1 & -1 & 1 & -1 & 1 & -1 \\
1 & -1 & 1 & -1 & -1 & 1 & -1 & 1 \\
1 & -1 & -1 & 1 & 1 & -1 & -1 & 1 \\
1 & -1 & -1 & 1 & -1 & 1 & 1 & -1
\end{array}\right) .
$$

From (4.19), they give 8 mutually commuting bosons $\boldsymbol{\varepsilon}_{(n)} \cdot \boldsymbol{\phi}_{y} / 2$ per wire

$$
\begin{aligned}
& {\left[\frac{1}{2} \boldsymbol{\varepsilon}_{(m)} \cdot \boldsymbol{\phi}_{y}(x, t), \frac{1}{2} \boldsymbol{\varepsilon}_{(n)} \cdot \boldsymbol{\phi}_{y^{\prime}}\left(x^{\prime}, t\right)\right]} \\
& =2 \pi i \delta_{m n}(-1)^{y} \delta_{y y^{\prime}} \operatorname{sgn}\left(x^{\prime}-x\right)
\end{aligned}
$$

up to a constant integral multiple of $2 \pi i$.

We separate the 8 vectors into two groups $\mathcal{S}^{+}=\left\{\boldsymbol{\varepsilon}_{(1)}, \boldsymbol{\varepsilon}_{(2)}, \boldsymbol{\varepsilon}_{(3)}, \boldsymbol{\varepsilon}_{(4)}\right\}$ and $\mathcal{S}^{-}=$ $\left\{\boldsymbol{\varepsilon}_{(5)}, \boldsymbol{\varepsilon}_{(6)}, \boldsymbol{\varepsilon}_{(7)}, \boldsymbol{\varepsilon}_{(8)}\right\}$. They defines the two $\widetilde{s o(8)_{1}^{ \pm}}$subalgebras in $E_{8}$, whose roots lie in the root lattice of $E_{8}$ orthogonal to $\mathcal{S}^{\mp}$ respectively. One could pick the simple roots

$$
\begin{aligned}
& \widetilde{\boldsymbol{\alpha}}_{1}^{+}=\boldsymbol{\varepsilon}_{(1)} / 2, \widetilde{\boldsymbol{\alpha}}_{2}^{+}=\mathbf{e}_{1}+\mathbf{e}_{2}, \widetilde{\boldsymbol{\alpha}}_{2}^{+}=\mathbf{e}_{3}+\mathbf{e}_{4}, \widetilde{\boldsymbol{\alpha}}_{4}^{+}=\mathbf{e}_{5}+\mathbf{e}_{6} \\
& \widetilde{\boldsymbol{\alpha}}_{1}^{-}=\boldsymbol{\varepsilon}_{(5)} / 2, \widetilde{\boldsymbol{\alpha}}_{2}^{-}=\mathbf{e}_{2}-\mathbf{e}_{1}, \widetilde{\boldsymbol{\alpha}}_{2}^{-}=\mathbf{e}_{4}-\mathbf{e}_{3}, \widetilde{\boldsymbol{\alpha}}_{4}^{-}=\mathbf{e}_{6}-\mathbf{e}_{5}
\end{aligned}
$$

so that their inner product recover the Cartan matrix of so(8)

$$
\widetilde{\boldsymbol{\alpha}}_{I}^{ \pm} \cdot \widetilde{\boldsymbol{\alpha}}_{J}^{ \pm}=K_{I J}, \quad K=\left(\begin{array}{cccc}
2 & -1 & -1 & -1 \\
-1 & 2 & 0 & 0 \\
-1 & 0 & 2 & 0 \\
-1 & 0 & 0 & 2
\end{array}\right)
$$

while opposite sectors decouple $\widetilde{\boldsymbol{\alpha}}_{I}^{ \pm} \cdot \widetilde{\boldsymbol{\alpha}}_{J}^{\mp}=0$. 
The new gapping potential is constructed by backscattering the two decoupled $\widetilde{s o(8)_{1}^{ \pm}}$currents to adjacent wires in opposite directions.

$$
\mathcal{H}_{\mathrm{int}}=u \sum_{y=-\infty}^{\infty} \mathbf{J}_{s o(8)_{1}^{-}}^{y} \cdot \mathbf{J}_{s o(8)_{1}^{+}}^{y+1}
$$

However not every terms can be written down as 4-fermion interactions. In particular $\mathcal{H}_{\text {int }}$ contains interwire $s_{+}$quasiparticle backscattering

$$
V_{y}^{\varepsilon} V_{y+1}^{-\varepsilon^{\prime}}+h . c . \sim \cos \left(\sum_{j=1}^{8} \frac{\varepsilon_{j}}{2} \tilde{\phi}_{y}^{j}-\frac{\varepsilon_{j}^{\prime}}{2} \tilde{\phi}_{y+1}^{j}\right)
$$

for $\varepsilon_{j}, \varepsilon_{j}^{\prime}= \pm 1$, that condenses pairs of $s_{+}$'s along adjacent wires and confines all electronic excitations. The $\widetilde{s o(8)_{1}^{ \pm}}$WZW CFT carries three emergent fermionic primary fields

$$
\widetilde{c}_{p}^{ \pm}=\exp \left[\frac{i}{2}\left(\tilde{\phi}^{2 p-1} \pm \tilde{\phi}^{2 p}-\tilde{\phi}^{7} \mp \tilde{\phi}^{8}\right)\right]
$$

for $p=1,2,3$. All of which have neutral electric charge and even fermion parity with respect to the original electronic operators $c_{j}=e^{i \tilde{\phi}^{j}}$. This is because the $\widetilde{c}_{p}^{ \pm}$'s are invariant under the $U(1)$ gauge transformation $\tilde{\phi}^{j} \rightarrow \tilde{\phi}^{j}+\varphi$. As a result, the interaction $(4.175)$ corresponds to a gapped $\widetilde{S O(8)_{1}}$ topological order but contains no electron-like anyon excitations. Lastly we notice that this matches with the surface topological order of a type-II topological paramagnet.[129, 111]

\subsubsection{Alternative conformal embeddings}

The fractionalization $s o(9)_{1} \supseteq s o(3)_{3} \otimes s o(3)_{3}$ in 4.2 .2 .1 is the corner stone for the construction of symmetric gapping interactions when there is an odd number of Majorana species. However, this is not the unique decomposition. In general when 
the number of Majorana channels is a whole square, the wire can be bipartitioned into $s o\left(n^{2}\right)_{1} \supseteq s o(n)_{n} \otimes s o(n)_{n} \cdot[26,11,108,10]$

For instance, this provides yet another alternative when $N=16$ where each wire is fractionalized into a pair of $s o(4)_{4}=s u(2)_{4} \times s u(2)_{4}$. The $s o(4)_{4}^{ \pm}$current operators can be constructed in a similar fashion as those in the $s o(3)_{3}^{ \pm}$case, $\mathbf{J}=\frac{i}{2} \boldsymbol{\Sigma}_{a b}^{ \pm} \psi^{a} \psi^{b}$ for $\boldsymbol{\Sigma}^{+}=\boldsymbol{\Sigma} \otimes \mathbb{1}_{4}$ and $\boldsymbol{\Sigma}^{-}=\mathbb{1}_{4} \otimes \boldsymbol{\Sigma}$ where $\boldsymbol{\Sigma}$ are antisymmetric $4 \times 4$ matrices generating so(4). After introducing the current-current backscattering interactions $\mathbf{J}_{s o(4)_{4}^{-}}^{y} \cdot \mathbf{J}_{s o(4)_{4}^{+}}^{y+1}$, the surface would carry a $S O(4)_{4}=S U(2)_{4} \times S U(2)_{4}$ topological order. Each $S U(2)_{4}$ theory contains five anyon types $j=\mathbf{0}, \mathbf{1} / \mathbf{2}, \mathbf{1}, \mathbf{3} / \mathbf{2}, \mathbf{2}$ with spins $h_{j}=j(j+1) / 6$. The $S O(4)_{4}$ topological state does not carry fermionic excitations, and therefore, like the previous example in 4.4.1, this gapping potential also removes all electronic quasiparticle excitations.

The gapped symmetric states for $N$ odd are not unique either. For example, the decomposition $s o(25)_{1} \supseteq s o(5)_{5} \otimes s o(5)_{5}$ leads to a surface $S O(5)_{5}$ topological order which is inequivalent to $G_{25}=S O(3)_{3} \otimes_{b} S O(8)_{1}$. 


\section{Chapter V}

\section{Conclusions and Outlooks}

In chapter I, we reviewed two important features of quantum many-body physics, entanglement and topology. We explained the motivation for studying the properties of scaling and classification respectively for these two concepts, both for theoretical consideration and potential applications. We then give an overview of the latest developments in related research directions and showed how our ideas to further improve those results are natural next steps.

In chapter II and III, we introduce a simple one dimensional spin model that features a state-of-the-art violation of the area law of entanglement entropy, resulting in a novel quantum phase transition in the meantime. Although we have acquired analytical results on both entanglement entropy and spectral gap, we are still far from understanding the order in the ground state or having a description for the low energy excitations. In addition to these obvious future directions, we mention a few others.

First, the commutation restriction observed in the proof of frustration freeness is interesting in itself, and one can explore whether similar extensions/deformations would work for other Hamiltonians written as sum of projectors, and if so, whether the same type of phase transition would occur. Second, while we have shown how to construct a multi-parameter deformation, we have only studied entropy and gap 
for a uniform parameter $t$. This choice keeps the chain translationally invariant, however, no momentum space arguments were involved in the analysis. A more general treatment will have to contend with the distribution of the $t_{A}$ parameters. Third, the nature of the quantum phase transition is unclear. To study the transition, as well as thermal effects, more detailed information about the density of states near the ground state is crucial. In particular, it would be very interesting to explore a possible field theoretic description in the continuous limit. Finally, it would be worthwhile to establish a proof on the gap in $0<t<1$ (for any $s$ ) phase observed in the numerical results given in ref. [12].

In chapter IV, we constructed a coupled Majorana wire model in $(2+1) \mathrm{D}$ that imitates the massless Majorana modes on the surface of a topological superconductor. This model had a non-local "antiferromagnetic" time reversal symmetry and consequently was $\mathbb{Z}_{2}$ classified - rather than $\mathbb{Z}$ in the class DIII TSC case - under the single-body framework. Despite the difference, this model adequately described the surface behavior of a TSC when the number $N$ of Majorana species was odd, and it was worth studying and interesting in and of itself.

We introduced the 4-fermion gapping potentials in section 4.2. They relied on the fractionalization or bipartition of the $s o(N)_{1}$ current along each wire into a pair of $\mathcal{G}_{N}$ channels (see eq.(4.33) and (4.34)). The two fractional channels then were backscattered onto adjacent wires in opposite directions. This froze all low energy degrees of freedom and opened an excitations energy gap without breaking time reversal symmetry. When $N=2 r$ was even, each wire could simply be split into a pair of $\mathcal{G}_{N}=s o(r)_{1}$ channels. The fractionalization was not as obvious when $N$ was odd. We first made use of the conformal embedding that decomposed nine Majorana's into two subsectors, $s o(9)_{1} \supseteq s o(3)_{3} \otimes s o(3)_{3}$ (see section 4.2.2.1). This division could be generalized by all odd cases by splitting a subset of 9 Majorana's into a pair of $s o(3)_{3}$ and the remaining even number of Majorana's into a pair of $s o(r)_{1}$. This could 
even be applied when $N$ is less then 9 because each wire could be reconstructed by adding an arbitrary number of helical Majorana modes with the same number of right and left movers.

The surface $G_{N}$ topological ordered was inferred from the bulk-boundary correspondence (see eq.(4.115)). These topological states followed a 32-fold periodicity $G_{N} \cong G_{N+32}$ and a relative tensor product structure $G_{N_{1}} \otimes_{b} G_{N_{2}} \cong G_{N_{1}+N_{2}}$. We presented the quasiparticle types as well as their fusion and braiding statistics properties. We explained the relative tensor product structure using the notion of anyon condensation[9]. On a more fundamental level, one should be able to deduce the topological order without the knowledge of the boundary by studying the modular properties of the degenerate bulk ground states under a compact torus geometry[134], or by directly looking at exchange and braiding behaviors of bulk excitations. In fact the coupled wire construction provided a fitting model for this purpose. Being an exactly solvable model, a ground state could be explicitly expressed as entangled superposition of tensor product ground states between each pair of wires. In the simplest case when the model is bosonizable, a ground state could be specified by the pinned angle variables of a collection of sine-Gordon potentials. The bulk excitations could be realized as kinks between a pair of wires and could be created by vertex operators. The virtue of a bulk description is that the action of time reversal on quasiparticle excitations could be examined explicitly, which we have not performed or addressed here. These issues are beyond the scope of this article and we refer a more detail discussion to subsequent works.

We noticed that there were alternative ways of fractionalization that led to different gapping interactions and consequently different topological orders. We saw in section 4.2.1.2 that $N=4$ was an exceptional case that requires the special bipartition $s o(4)_{1} \supseteq s u(2)_{1} \times s u(2)_{1}$ instead of two copies of $s o(2)_{1}$. We also saw in section 4.4 that when $N=16$, the surface could be gapped by alternative interactions that corre- 
sponded to a $\widetilde{S O(8)_{1}}$ or $S O(4)_{4}$ topological order, none of which contained electronic quasiparticle excitations. Other conformal embeddings $s o\left(n^{2}\right)_{1} \supseteq s o(n)_{n} \otimes s o(n)_{n}$ could give rise to multiple possibilities. Our 32-fold topological states, which only utilized $s o(9)_{1} \supseteq s o(3)_{3} \otimes s o(3)_{3}$, therefore should belong into a wider universal framework. These should be addressed in future works.

We conclude by pointing out that although not explored here, it will be interesting to study the topological aspects of the phases in the (deformed) Motzkin and Fredkin spin chains, an the entanglement properties in the topological order of TR symmetric gapped Majorana surface. 


\section{Appendix A}

\section{The $s o(N)$ Lie algebra and its representations}

The $s o(N)$ Lie algebra are generated by real antisymmetric matrices $t^{(r s)}=$ $\left(t_{a b}^{(r s)}\right)_{N \times N}$ with entries

$$
t_{a b}^{(r s)}=\delta_{a}^{r} \delta_{b}^{s}-\delta_{b}^{r} \delta_{a}^{s}
$$

for $r, s=1, \ldots, N$. There are $N(N-1) / 2$ linearly independent generators since $t^{(r s)}=-t^{(s r)}$ and $t^{(r r)}=0$. In the main text, we write the basis labels as $\beta=(r s)$, for $r<s$, for conciseness. The generators obey the commutator relation

$$
\left[t^{(r s)}, t^{(p q)}\right]=\sum_{m<n} f_{(r s)(p q)(m n)} t^{(m n)}
$$

where the structure constant is

$$
\begin{aligned}
f_{(r s)(p q)(m n)}= & \delta_{m r} \delta_{n q} \delta_{s p}-\delta_{m r} \delta_{n p} \delta_{s q} \\
& +\delta_{m s} \delta_{r q} \delta_{n p}-\delta_{m s} \delta_{n q} \delta_{r p}
\end{aligned}
$$

The matrix representation (A.1) is referred as the fundamental representation of $s o(N)$ and is labeled by $\psi$. In general the generators of $s o(N)$ can have different 
irreducible matrix representations $t_{\lambda}^{(r s)}=t_{\lambda}^{\beta}$ labeled by $\lambda$. Since the quadratic Casmir operator

$$
\hat{\mathcal{Q}}_{\lambda}=-\sum_{\beta} t_{\lambda}^{\beta} t_{\lambda}^{\beta}
$$

commutes with all the generators, it must have a fixed eigenvalue $\mathcal{Q}_{\lambda}$ that (incompletely) characterizes the irreducible representation $\lambda$. For instance, the fundamental representation in (A.1), denoted by $\psi$, has quadratic Casmir value $\mathcal{Q}_{\psi}=N-1$.

The spinor representation $\sigma$ of $s o(N)$ makes use of the Clifford algebra[67] $\left\{\gamma_{a}, \gamma_{b}\right\}=\gamma_{a} \gamma_{b}+\gamma_{b} \gamma_{a}=2 \delta_{a b}$ where $\gamma_{1}, \ldots, \gamma_{N}$ are hermitian matrices of dimension $d=2^{N / 2}$ for $N$ even or $d=2^{(N-1) / 2}$ for $N$ odd. The $s o(N)$ generators are represented as the quadratic combination

$$
t_{\sigma}^{(r s)}=\frac{1}{4} \sum_{a b} \gamma_{a} t_{a b}^{(r s)} \gamma_{b}=\frac{1}{2} \gamma_{r} \gamma_{s}
$$

and satisfy (A.2). When $N$ is even, the parity operator $(-1)^{F}=i^{N / 2} \gamma_{1} \ldots \gamma_{N}$ commutes with all $t_{\sigma}^{(r s)}$ and the representation is decomposable into $\sigma=s_{+} \oplus s_{-}$, where $s_{ \pm}$are $2^{N / 2-1}$-dimensional sectors with $(-1)^{F}= \pm 1$. The $s o(N)$ generators are then irreducibly represented by

$$
t_{s_{ \pm}}^{(r s)}=P_{ \pm} t_{\sigma}^{(r s)} P_{ \pm}^{\dagger}
$$

where $P_{ \pm}$are the projection operators onto the fixed parity subspaces. As $t_{\sigma}^{(r s)} t_{\sigma}^{(r s)}=$ $-(1 / 4) \mathbb{1}$, the quadratic Casmir values (A.4) of spinor representations are

$$
\mathcal{Q}_{\sigma}=\frac{N(N-1)}{8}, \quad \mathcal{Q}_{s_{ \pm}}=\frac{N(N-1)}{8}
$$


The complexified $s o(N)$ Lie algebra has an alternative set of Cartan-Weyl generators. It consists of a maximal set of commuting hermitian generators $H^{1}, \ldots, H^{r}$, and a finite set of raising of lowering operators $E^{\boldsymbol{\alpha}}=\left(E^{-\boldsymbol{\alpha}}\right)^{\dagger}$, labeled by integral vectors $\boldsymbol{\alpha}=\left(\alpha^{1}, \ldots, \alpha^{r}\right) \in \Delta$ called roots. The root lattice is given by the set

$$
\begin{aligned}
\Delta_{s o(2 r)} & =\left\{ \pm \mathbf{e}_{I} \pm \mathbf{e}_{J}: 1 \leq I<J \leq r\right\} \\
\Delta_{s o(2 r+1)} & =\Delta_{s o(2 r)} \cup\left\{ \pm \mathbf{e}_{I}: 1 \leq I \leq r\right\}
\end{aligned}
$$

where $\mathbf{e}_{I}$ are unit basis vectors of $\mathbb{R}^{r}$. In particular, there are $r$ simple roots $\boldsymbol{\alpha}_{1}, \ldots, \boldsymbol{\alpha}_{r}$ that forms a basis for the root lattice. For $s o(N)$ they can be chosen to be

$$
\boldsymbol{\alpha}_{I}= \begin{cases}\mathbf{e}_{I}-\mathbf{e}_{I+1}, & \text { for } I=1, \ldots, r-1 \\ \mathbf{e}_{r}, & \text { for } I=r \text { and } N \text { odd } \\ \mathbf{e}_{r-1}+\mathbf{e}_{r}, & \text { for } I=r \text { and } N \text { even }\end{cases}
$$

The set of roots $\Delta$ consists of integral combinations of the simple roots $\boldsymbol{\alpha}=$ $\sum_{J=1}^{r} b^{J} \boldsymbol{\alpha}_{J}$ so that its length is $|\boldsymbol{\alpha}|=\sqrt{2}$, for even $N$, or $|\boldsymbol{\alpha}|=1$ or $\sqrt{2}$, for odd $N$.

The integer $r$ is the rank of the $s o(N)$ Lie algebra and is determined by $N=2 r$ for $N$ even or $N=2 r+1$ for $N$ odd. These generators satisfy

$$
\begin{gathered}
{\left[H^{i}, E^{\boldsymbol{\alpha}}\right]=\alpha^{i} E^{\boldsymbol{\alpha}}, \quad\left[E^{\boldsymbol{\alpha}}, E^{-\boldsymbol{\alpha}}\right]=\frac{2}{|\boldsymbol{\alpha}|^{2}} \sum_{i=1}^{r} \alpha^{i} H^{i}} \\
{\left[E^{\boldsymbol{\alpha}}, E^{\boldsymbol{\beta}}\right] \propto\left\{\begin{array}{cc}
E^{\boldsymbol{\alpha}+\boldsymbol{\beta}}, & \text { if } \boldsymbol{\alpha}+\boldsymbol{\beta} \in \Delta \\
0, & \text { if otherwise }
\end{array}, \text { for } \boldsymbol{\alpha} \neq \boldsymbol{\beta} .\right.}
\end{gathered}
$$

The Cartan matrix $K=\left(K_{I J}\right)_{r \times r}$ of the algebra is defined by the scalar product

$$
K_{I J}=\frac{2 \boldsymbol{\alpha}_{I}^{T} \boldsymbol{\alpha}_{J}}{\left|\boldsymbol{\alpha}_{J}\right|^{2}}=\sum_{i=1}^{r} \frac{2 \alpha_{I}^{i} \alpha_{J}^{i}}{\left|\boldsymbol{\alpha}_{J}\right|^{2}}
$$


so $(2 r)$ is simply-laced in the sense that all roots have identical length and the Cartan matrix is therefore symmetric

$$
K_{s o(2 r)}=\left(\begin{array}{ccccc}
2 & -1 & 0 & \ldots & 0 \\
-1 & 2 & \ddots & & \vdots \\
0 & \ddots & 2 & -1 & -1 \\
\vdots & & -1 & 2 & 0 \\
0 & \ldots & -1 & 0 & 2
\end{array}\right)
$$

Sometimes it would be convenient to use the Chevalley basis so that the commuting generators are redefined

$$
h^{I}=\frac{2}{\left|\boldsymbol{\alpha}_{I}\right|^{2}} \sum_{i=1}^{r} \alpha_{I}^{i} H^{i}
$$

so that the commutator relations (A.10) becomes

$$
\left[h^{I}, E^{ \pm \boldsymbol{\alpha}_{J}}\right]= \pm K_{I J} E^{ \pm \boldsymbol{\alpha}_{J}}, \quad\left[E^{\boldsymbol{\alpha}_{J}}, E^{-\boldsymbol{\alpha}_{J}}\right]=\delta^{I J} h^{J}
$$




\section{Appendix B}

\section{Bosonizing the $s o(2 r)_{1}$ current algebra}

Here we review the bosonization[26, 140, 36] of a chiral wire with $N=2 r$ Majorana fermions, and express the $s o(2 r)_{1}$ current operators in bosonized form. The $2 r$ Majorana (real) fermions can be paired into $r$ Dirac (complex) fermions and bosonized into the normal ordered vertex operators

$$
c^{j}(z)=\frac{\psi^{2 j-1}(z)+i \psi^{2 j}(z)}{\sqrt{2}} \sim \exp \left(i \widetilde{\phi}^{j}(z)\right) .
$$

Here we focus on a single wire, say at an even $y$, so that all fields depend on the holo-

morphic parameter $z=e^{\tau+i x}$. The $r$-component boson $\widetilde{\phi}=\left(\widetilde{\phi}^{1}, \ldots, \widetilde{\phi}^{r}\right)$ is governed by the Lagrangian density

$$
\mathcal{L}_{0}=\frac{1}{2 \pi} \sum_{j=1}^{r} \partial_{x} \widetilde{\phi}^{j} \partial_{t} \widetilde{\phi}^{j}=\frac{1}{2 \pi} \partial_{x} \widetilde{\phi} \partial_{t} \widetilde{\boldsymbol{\phi}}
$$

and follows the algebraic relations

$$
\left[\widetilde{\phi}^{j}(x, t), \widetilde{\phi}^{j^{\prime}}\left(x^{\prime}, t\right)\right]=i \pi\left[\delta^{j j^{\prime}} \operatorname{sgn}\left(x^{\prime}-x\right)+\operatorname{sgn}\left(j-j^{\prime}\right)\right]
$$


or equivalently the time-ordered correlation function

$$
\left\langle\widetilde{\phi}^{j}(z) \widetilde{\phi}^{j^{\prime}}(w)\right\rangle=-\delta^{j j^{\prime}} \log (z-w)+\frac{i \pi}{2} \operatorname{sgn}\left(j-j^{\prime}\right)
$$

for $\operatorname{sgn}(s)=s /|s|$ when $s \neq 0$ and $\operatorname{sgn}(0)=0$. Operator product expansions between unordered vertex operators can be evaluated by $e^{A(z)} e^{B(w)}=e^{A(z)+B(w)+\langle A(z) B(w)\rangle}$, for $A, B$ linear combination of the bosons $\widetilde{\phi}^{j}$. For instance, the vertex operators in (B.1) reproduce the product expansion of a pair of identical Dirac fermions

$$
c^{j}(z)\left(c^{j}(w)\right)^{\dagger}=\frac{1}{z-w}+i \partial \widetilde{\phi}^{j}(w)+\ldots
$$

and the singular piece is dropped when the product is normal ordered in the limit $z \rightarrow w$. The non-singular sign factor $i \pi \operatorname{sgn}\left(j-j^{\prime}\right)$ ensures fermions with distinct flavors anticommutes

$$
c^{j}(z) c^{j^{\prime}}(w)=-c^{j^{\prime}}(w) c^{j}(z)
$$

The $s o(2 r)_{1}$ currents in the Cartan-Weyl basis can now be bosonized

$$
\begin{aligned}
& H^{j}(z)=c^{j}(z) c^{j}(z)^{\dagger}=i \partial_{z} \widetilde{\phi}^{j}(z) \\
& E^{\boldsymbol{\alpha}}(z)=\prod_{j=1}^{r} c^{j}(z)^{\alpha^{j}}=\exp (i \boldsymbol{\alpha} \cdot \widetilde{\boldsymbol{\phi}}(z))
\end{aligned}
$$

where $\boldsymbol{\alpha}=\left(\alpha^{1}, \ldots, \alpha^{r}\right) \in \Delta$ are roots of $s o(2 r)$ (see (A.8)) and the fermion products are normal ordered. For instance, $\boldsymbol{\alpha}$ has two and only two non-zero entries and $E^{\boldsymbol{\alpha}}$ must be of the form

$$
E^{\alpha}(z)=c^{i}(z)^{ \pm} c^{j}(z)^{ \pm}=e^{i\left( \pm \widetilde{\phi}^{i}(z) \pm \widetilde{\phi}^{j}(z)\right)}
$$


Combining raising or lowering operators give

$$
E^{\boldsymbol{\alpha}}(z) E^{\boldsymbol{\beta}}(w)=i^{-\boldsymbol{\alpha} \cdot \boldsymbol{\beta}} \epsilon(\boldsymbol{\alpha}, \boldsymbol{\beta}) \frac{e^{i(\boldsymbol{\alpha} \cdot \tilde{\boldsymbol{\phi}}(z)+\boldsymbol{\beta} \cdot \tilde{\boldsymbol{\phi}}(w))}}{(z-w)^{-\boldsymbol{\alpha} \cdot \boldsymbol{\beta}}}
$$

where the vertex operator here is again normal ordered and the 2-cocyle is given by the star product

$$
\epsilon(\boldsymbol{\alpha}, \boldsymbol{\beta})=(-1)^{\boldsymbol{\alpha} * \boldsymbol{\beta}}=(-1)^{\sum_{i>j} \alpha^{i} \beta^{j}}
$$

As $\sum_{i=1}^{r} \alpha^{i}$ is even for all roots, we have the following simplification when interchang$\operatorname{ing} \boldsymbol{\alpha} \leftrightarrow \boldsymbol{\beta}$

$$
\epsilon(\boldsymbol{\alpha}, \boldsymbol{\beta}) \epsilon(\boldsymbol{\beta}, \boldsymbol{\alpha})=(-1)^{\boldsymbol{\alpha} \cdot \boldsymbol{\beta}}
$$

Using the boson OPE (B.4), the product of the two vertex operators above is singular only when (i) $\boldsymbol{\alpha}=-\boldsymbol{\beta}$, or (ii) $\boldsymbol{\alpha} \cdot \boldsymbol{\beta}=-1$ in other words $\boldsymbol{\alpha}+\boldsymbol{\beta} \in \Delta$. To summarize, the Cartan-Weyl generators satisfy the product expansion

$$
\begin{aligned}
H^{i}(z) H^{j}(w) & =\frac{\delta^{i j}}{(z-w)^{2}}-\partial \widetilde{\phi}^{i}(w) \partial \widetilde{\phi}^{j}(w)+\ldots \\
H^{i}(z) E^{\boldsymbol{\alpha}}(w) & =\frac{\alpha^{i}}{z-w} E^{\boldsymbol{\alpha}}(w)+\ldots \\
E^{\boldsymbol{\alpha}}(z) E^{-\boldsymbol{\alpha}}(w) & =\frac{1}{(z-w)^{2}}+\sum_{i=1}^{r} \frac{\alpha^{i}}{z-w} H^{i}(w) \\
& -\frac{1}{2}(\boldsymbol{\alpha} \cdot \partial \widetilde{\boldsymbol{\phi}}(w))^{2}+\ldots \\
E^{\boldsymbol{\alpha}}(z) E^{\boldsymbol{\beta}}(w) & =\frac{i \epsilon(\boldsymbol{\alpha}, \boldsymbol{\beta})}{z-w} E^{\boldsymbol{\alpha}+\boldsymbol{\beta}}(w)+\ldots, \quad \text { if } \boldsymbol{\alpha} \cdot \boldsymbol{\beta}=-1 .
\end{aligned}
$$

For instance, the 2-cocyle coefficient $\epsilon(\boldsymbol{\alpha}, \boldsymbol{\beta})$ ensures the OPE between $E^{\boldsymbol{\alpha}}(z)$ and $E^{\boldsymbol{\beta}}(w)$ commute as the sign in (B.11) when exchanging $\boldsymbol{\alpha} \leftrightarrow \boldsymbol{\beta}$ cancels that in $1 /(z-w)$ when switching $z \leftrightarrow w$. 
In certain derivations, especially when involving quasiparticle excitations, it may be more convenient to use the Chevalley basis. Here fields are expressed in terms of non-local bosons $\phi=\left(\phi^{1}, \ldots, \phi^{r}\right)$, which are related to the original ones by the (non-unimodular) basis transformation

$$
\widetilde{\phi}^{i}=\sum_{I=1}^{r} \alpha_{I}^{i} \phi^{I}
$$

using the simple roots $\boldsymbol{\alpha}_{I}=\left(\alpha_{I}^{1}, \ldots, \alpha_{I}^{r}\right) \in \mathbb{Z}^{r}$ (see (A.9) in appendix A). The Lagrangian density (B.2) now becomes

$$
\mathcal{L}_{0}=\frac{1}{2 \pi} \sum_{I, J=1}^{r} K_{I J} \partial_{x} \phi^{I} \partial_{t} \phi^{J}
$$

where $K=\left(K_{I J}\right)_{r \times r}=\boldsymbol{\alpha}_{I} \cdot \boldsymbol{\alpha}_{J}$ is the Cartan matrix of $s o(2 r)_{1}$ (see eq.(A.12)).

The current generators are rewritten in the Chevalley basis by

$$
\begin{aligned}
h_{I}(z) & =\sum_{i=1}^{r} \alpha_{I}^{i} H^{i}(z)=i \sum_{J=1}^{r} K_{I J} \partial_{z} \phi^{J}(z) \\
E^{\mathbf{b}}(z) & =E^{\boldsymbol{\beta}}(z)=\exp \left(i \mathbf{b}^{T} K \boldsymbol{\phi}^{J}(z)\right)
\end{aligned}
$$

where $\boldsymbol{\beta}=\sum_{J} b^{J} \boldsymbol{\alpha}_{J}$ are roots expressed in integral combinations of the simple ones, for $\mathbf{b}=\left(b^{1}, \ldots, b^{r}\right) \in \mathbb{Z}^{r}$. The Chevalley generators satisfy the modified current relations from (B.12)

$$
\begin{aligned}
h_{I}(z) h_{J}(w) & =\frac{K_{I J}}{(z-w)^{2}}+\ldots \\
h_{I}(z) E^{\mathbf{b}}(w) & =\frac{K_{I J} b^{J}}{z-w} E^{\mathbf{b}}(w)+\ldots \\
E^{\mathbf{b}}(z) E^{-\mathbf{b}}(w) & =\frac{1}{(z-w)^{2}}+\sum_{I=1}^{r} \frac{b^{I}}{z-w} h_{I}(w)+\ldots \\
E^{\mathbf{b}_{1}}(z) E^{\mathbf{b}_{2}}(w) & =\frac{i \epsilon\left(\boldsymbol{\beta}_{1}, \boldsymbol{\beta}_{2}\right)}{z-w} E^{\mathbf{b}_{1}+\mathbf{b}_{2}}(w)+\ldots
\end{aligned}
$$


if $\mathbf{b}_{1}^{T} K \mathbf{b}_{2}=-1$.

The (normal ordered) energy-momentum tensor can be turned from the Sugawara form (4.11) to the usual bosonic one

$$
\begin{aligned}
T(z) & =\frac{1}{2(N-1)}\left[\sum_{i=1}^{r} H^{i}(z) H^{i}(z)+\sum_{\boldsymbol{\alpha} \in \Delta} E^{\alpha}(z) E^{-\alpha}(z)\right] \\
& =-\frac{1}{2} \partial \widetilde{\phi}(z) \cdot \partial \widetilde{\phi}(z)=-\frac{1}{2} \partial \phi(z) \cdot K \partial \phi(z)
\end{aligned}
$$

Excitations in the CFT can be easily represented by vertex operators

$$
V^{\mathbf{a}}(z)=\exp (i \mathbf{a} \cdot \boldsymbol{\phi}(z))=\exp \left(i \mathbf{a}_{\vee} \cdot \widetilde{\phi}(z)\right)
$$

labeled by integral lattice vectors $\mathbf{a}=\left(a_{1}, \ldots, a_{r}\right)$, or equivalently dual root lattice vectors $\mathbf{a}_{\vee}=\left(a_{\vee}^{1}, \ldots, a_{\vee}^{r}\right)$ with rational entries

$$
a_{\vee}^{j}=\sum_{I J} a_{I}\left(K^{-1}\right)^{I J} \alpha_{J}^{j}
$$

The conformal dimension of $V^{\mathbf{a}}$ can be read off by the inner product

$$
\begin{aligned}
h_{\mathbf{a}} & =\frac{1}{2} \mathbf{a}^{T} K^{-1} \mathbf{a}=\frac{1}{2}\left(K^{-1}\right)^{I J} a_{I} a_{J} \\
& =\frac{1}{2} \mathbf{a}_{\vee}^{T} \mathbf{a}_{\vee}=\frac{1}{2} \delta_{i j} a_{\vee}^{i} a_{\vee}^{j} .
\end{aligned}
$$

This can be evaluated from definition (4.15) using the energy-momentum tensor (B.17) and the OPE

$$
\partial_{z} \phi_{I}(z) \phi_{J}(w)=-\left(K^{-1}\right)^{I J} \log (z-w)+\ldots
$$

which is equivalent to (B.4). 
Most vertex operators (B.18) however are not WZW primary and do not represent the $s o(2 r)_{1}$ Kac-Moody algebra. The OPE with the current generators

$$
\begin{aligned}
h_{I}(z) V^{\mathbf{a}}(w) & =\frac{a_{I}}{z-w} V^{\mathbf{a}}(w)+\ldots \\
E^{\mathbf{b}}(z) V^{\mathbf{a}}(w) & =c_{\mathbf{a}}^{\mathbf{b}}(z-w)^{\mathbf{a} \cdot \mathbf{b}} V^{\mathbf{a}+K \mathbf{b}}(w)+\ldots
\end{aligned}
$$

would match the requirement (4.13) for a primary field only when the exponent of the singular term is bounded below, i.e. $\mathbf{a} \cdot \mathbf{b} \geq-1$ for all roots $\boldsymbol{\beta}=\sum_{I} b^{I} \boldsymbol{\alpha}_{I}$. Such lattice vectors a are called weights or Dynkin labels of $s o(2 r)$ at level 1 . When the exponenet $\mathbf{a} \cdot \mathbf{b}$ in (B.22) is -1 , the vertex operators $V^{\mathbf{a}}$ and $V^{\mathbf{a}+K \mathbf{b}}$ are related by the $S O(2 r)_{1}$ symmetry and belong to the same primary field sector. For example the unit vector $\mathbf{a}=\mathbf{e}_{1}$ is the highest weight that generates the fermion sector $\psi$. Applying lowering operators $E^{-\mathbf{b}}$ to $V^{\mathbf{e}_{1}}=c^{1}$ gives all $2 r$ Dirac fermions

$$
\mathbf{V}_{\psi}=\operatorname{span}\left\{\left(c^{j}\right)^{ \pm}=e^{ \pm i \widetilde{\phi}^{j}}: j=1, \ldots, r\right\}
$$

which in turn irreducibly represent the $s o(2 r)_{1}$ algebra (see (4.13)) according to the fundamental vector representation.

The unit vectors $\mathbf{a}=\mathbf{e}_{r-1}$ and $\mathbf{e}_{r}$ generate the two spinor sectors $s_{-}$and $s_{+}$ respectively. Each of them consists of $2^{r-1}$ twist fields

$$
\begin{aligned}
\mathbf{V}_{s_{ \pm}} & =\sigma^{1} \ldots \sigma^{2 r} \\
& =\operatorname{span}\left\{\exp \left(i \sum_{j=1}^{r} \frac{(-1)^{s_{j}}}{2} \widetilde{\phi}^{j}\right): \prod_{j=1}^{r}(-1)^{s_{j}}= \pm 1\right\} .
\end{aligned}
$$

They irreducibly represent the $s o(2 r)_{1}$ algebra according to the even and odd spinor representations. These are the only primary fields of $s o(2 r)_{1}$ and their conformal dimensions are given by $h_{\psi}=1 / 2$ and $h_{s_{ \pm}}=r / 8$. 
The four primary fields $1, \psi, s_{ \pm}$obey a set of fusion rules, which are OPE keeping only primary fields.

$$
\begin{gathered}
s_{ \pm} \times \psi=s_{\mp} \\
s_{ \pm} \times s_{ \pm}\left\{\begin{array}{ll}
1, & \text { for } r \text { even } \\
\psi, & \text { for } r \text { odd }
\end{array} \quad s_{ \pm} \times s_{\mp}\left\{\begin{array}{ll}
\psi, & \text { for } r \text { even } \\
1, & \text { for } r \text { odd }
\end{array} .\right.\right.
\end{gathered}
$$

For instance, the OPE

$$
\begin{aligned}
V_{s_{+}}(z) c^{r}(w)^{\dagger} & =e^{i \frac{\widetilde{\phi}^{1}(z)+\ldots+\tilde{\phi}^{r}(z)}{2}} e^{-i \widetilde{\phi}^{r}(w)} \\
& \propto(z-w)^{-\frac{1}{2}} e^{i \frac{\widetilde{\phi}^{1}(w)+\ldots+\tilde{\phi}^{r}(w)-\widetilde{\phi}^{r}(w)}{2}}+\ldots \\
& =(z-w)^{-\frac{1}{2}} V_{s_{-}}(w)+\ldots
\end{aligned}
$$

shows $s_{+} \times \psi=s_{-}$, and

$$
e^{i \sum_{j} \widetilde{\phi}^{j}(z) / 2} e^{-i \sum_{j} \widetilde{\phi}^{j}(w) / 2} \propto(z-w)^{-\frac{r}{4}}+\ldots
$$

shows $s_{+} \times s_{+}=1$ for $r$ even, or $s_{+} \times s_{-}=1$ for $r$ odd. 


\section{Appendix C}

\section{Bosonizing the $s o(2 r+1)_{1}$ current algebra}

A chiral wire with $N=2 r+1$ Majorana fermions can be partially bosonized by grouping $\psi^{1}, \ldots, \psi^{2 r}$ in pairs to form $r$ Dirac fermions (see (B.1)). This leaves a single Majorana $\psi^{2 r+1}$ behind. In order for the fermions to obey the correct anticommutation relations, the bosonized complex fermions (B.1) have to be modified by a Klein factor

$$
c^{j}(z)=(-1)^{\Pi} e^{i \widetilde{\phi}^{j}(z)}=e^{i \widetilde{\phi}^{j}(z)+i \pi \Pi}
$$

where $(-1)^{\Pi}$ is the fermion parity operator that anticommutes with $\psi^{2 r+1}$, and both $\Pi$ and $\psi_{2 r+1}$ commute with the rest of the bosons $\widetilde{\phi}^{j}$. In a non-chiral system, $(-1)^{\Pi}$ can be chosen to be the combination $i \gamma_{L} \gamma_{R}$, for $\gamma_{L / R}$ the zero mode of $\psi_{L / R}^{2 r+1}$. In the chiral case, it can be defined by $i \gamma \gamma_{\infty}$ using an additional Majorana zero mode $\gamma_{\infty}$ that completes the Clifford algebra $\left\{\gamma, \gamma_{\infty}\right\}=0$.

The $s o(2 r+1)_{1}$ current algebra extends the $s o(2 r)_{1}$ algebra by the short roots with length 1 (see (A.8)). It contains the $s o(2 r)_{1}$ generators $H^{j}=i \partial \widetilde{\phi}^{j}$ and $E^{\boldsymbol{\alpha}}=e^{i \boldsymbol{\alpha} \cdot \widetilde{\boldsymbol{\phi}}}$ (see (B.7) in appendix B), for $\boldsymbol{\alpha} \in \Delta_{s o(2 r)}$ the long roots with length $|\boldsymbol{\alpha}|=\sqrt{2}$. The remaining raising and lowering operators with the short roots are represented by the 
normal ordered products

$$
E^{ \pm \mathbf{e}_{j}}(z)=e^{ \pm i \widetilde{\phi}^{j}(z)} \psi^{2 r+1}(z)
$$

In addition to (B.12), the Cartan-Weyl generators satisfy the current relations

$$
\begin{aligned}
& H^{i}(z) E^{ \pm \mathbf{e}_{j}}(w)=\frac{ \pm \delta^{i j}}{z-w} E^{ \pm \mathbf{e}_{j}}(w)+\ldots \\
& E^{\mathbf{e}_{j}}(z) E^{-\mathbf{e}_{j}}(w)=\frac{1}{(z-w)^{2}}+\frac{1}{z-w} H^{j}(w) \\
& -\frac{1}{2} \partial \widetilde{\phi}^{j}(w) \partial \widetilde{\phi}^{j}(w) \\
& -\psi^{2 r+1}(w) \partial \psi^{2 r+1}(w)+\ldots \\
& E^{s_{1} \mathbf{e}_{j_{1}}}(z) E^{s_{2} \mathbf{e}_{j_{2}}}(w)=\frac{i^{-s_{1} s_{2}} \epsilon\left(\mathbf{e}_{j_{1}}, \mathbf{e}_{j_{2}}\right)}{z-w} E^{s_{1} \mathbf{e}_{j_{1}}+s_{2} \mathbf{e}_{j_{2}}}(w) \\
& +\ldots
\end{aligned}
$$

for $j_{1} \neq j_{2}$ and $s_{1}, s_{2}= \pm 1$. Moreover, when $\boldsymbol{\alpha} \cdot\left( \pm \mathbf{e}_{j}\right)=-1$, i.e. $\boldsymbol{\alpha} \pm \mathbf{e}_{j} \in \Delta_{s o(2 r+1)}$,

$$
E^{\boldsymbol{\alpha}}(z) E^{ \pm \mathbf{e}_{j}}(w)=\frac{i \epsilon\left(\boldsymbol{\alpha}, \mathbf{e}_{j}\right)(-1)^{\sum_{j} \alpha^{j} / 2}}{z-w} E^{\boldsymbol{\alpha} \pm \mathbf{e}_{j}}(w)+\ldots
$$

where $\epsilon(\mathbf{m}, \mathbf{n})=(-1)^{\mathbf{m} * \mathbf{n}}$ is defined in $($ B.10).

The (normal ordered) energy-momentum tensor can be turned from the Sugawara form (4.11) to the usual bosonic and fermionic one

$$
\begin{aligned}
T(z)= & \frac{1}{2(N-1)}\left[\sum_{i=1}^{r} H^{i}(z) H^{i}(z)+\sum_{\boldsymbol{\alpha} \in \Delta} E^{\boldsymbol{\alpha}}(z) E^{-\boldsymbol{\alpha}}(z)\right. \\
& \left.+\sum_{j=1}^{r} E^{\mathbf{e}_{j}}(z) E^{-\mathbf{e}_{j}}(z)+E^{-\mathbf{e}_{j}}(z) E^{\mathbf{e}_{j}}(z)\right] \\
=- & \frac{1}{2} \partial \widetilde{\boldsymbol{\phi}}(z) \cdot \partial \widetilde{\boldsymbol{\phi}}(z)-\frac{1}{2} \psi^{2 r+1}(z) \partial \psi^{2 r+1}(z) .
\end{aligned}
$$


There are only two non-trivial primary fields $\psi$ and $\sigma$. The fermion sector $\psi$ consists of the $2 r$ Dirac fermions $c^{j},\left(c^{j}\right)^{\dagger}$ in (B.23) as well as the remaining Majorana fermion $\psi^{2 r+1}$. The $\sigma$ sector consists of $2^{r}$ twist fields

$$
\begin{aligned}
\mathbf{V}_{\sigma} & =\sigma^{1} \ldots \sigma^{2 r+1} \\
& =\operatorname{span}\left\{\exp \left(i \sum_{j=1}^{r} \frac{(-1)^{s_{j}}}{2} \widetilde{\phi}^{j}\right) \sigma^{2 r+1}: s_{j}=0,1\right\}
\end{aligned}
$$

which represents $s o(2 r+1)_{1}$ according to the spinor representation. Their conformal dimensions are given by $h_{\psi}=1 / 2$ and $h_{\sigma}=(2 r+1) / 16$. 


\section{Appendix D}

\section{$\mathbb{Z}_{6}$ parafermion model}

Here we represent the $\mathbb{Z}_{6}$ parafermions using bosonized fields and Majorana fermions in the $s o(9)_{1} \mathrm{CFT}$. We focus on a single Majorana wire containing 9 right moving real fermions. The CFT is fractionalized using the conformal embedding into $s o(9)_{1} \supseteq s o(3)_{3}^{+} \times s o(3)_{3}^{-}$(see section 4.2.2.1). Each $s o(3)_{3}$ sector is then further decomposed into so $(2)_{3} \times$ " $\mathbb{Z}_{6}$ " using the coset construction " $\mathbb{Z}_{6}$ " $=s o(3)_{3} /$ so $(2)_{3}$ (see section 4.2.2.2). We now provide a more detail description of the $\mathbb{Z}_{6}$ parafermion sector. We will focus on the one in $s o(3)_{3}^{-}$.

First we pair six Majorana channels into three Dirac fermions and bosonize $c^{1}=$ $\left(\psi^{1}+i \psi^{4}\right) / \sqrt{2}=e^{i \widetilde{\phi}^{1}}, c^{2}=\left(\psi^{2}+i \psi^{5}\right) / \sqrt{2}=e^{i \widetilde{\phi}^{2}}$ and $c^{3}=\left(\psi^{3}+i \psi^{6}\right) / \sqrt{2}=e^{i \widetilde{\phi}^{3}}$. The Lagrangian density of the boson fields are given in (4.97). Like the $s o(N)_{1}$ case, extra care is required so that the Dirac fermions $c^{j}$ satisfies the appropriate mutual anticommutation relations. Here we use a slightly different but more convenient 
convention

$$
\begin{aligned}
\left\langle\widetilde{\phi}^{i}(z) \widetilde{\phi}^{j}(w)\right\rangle= & -\delta^{i j} \log (z-w)+\frac{i \pi}{2} S^{i j} \\
S^{i j} & =\left\{\begin{array}{cc}
0 & \text { if } i=j \\
1 & \text { if } i-j \equiv 1 \bmod 3 \\
-1 & \text { if } i-j \equiv-1 \bmod 3
\end{array}\right.
\end{aligned}
$$

so that the constant phases $S^{i j}$ have a threefold cyclic symmetry. The $s o(2)_{3}$ subtheory is generated by the "charged" boson $\phi_{\rho}=\left(\widetilde{\phi}^{1}+\widetilde{\phi}^{2}+\widetilde{\phi}^{3}\right) / 3$. It satisfies

$$
\left\langle\phi_{\rho}(z) \phi_{\rho}(w)\right\rangle=-\frac{1}{3} \log (z-w)
$$

The remaining "neutral" bosons $\phi_{\sigma}^{j}=\widetilde{\phi}^{j}-\phi_{\rho}$ are linearly dependent $\phi_{\sigma}^{1}+\phi_{\sigma}^{2}+\phi_{\sigma}^{3}=0$ and obey the OPE

$$
\left\langle\phi_{\sigma}^{i}(z) \phi_{\sigma}^{j}(w)\right\rangle=-\left(\delta^{i j}-\frac{1}{3}\right) \log (z-w)+\frac{i \pi}{2} S^{i j}
$$

The "charge" and "neutral" sector completely decoupled so that $\left\langle\phi_{\rho}(z) \phi_{\sigma}^{j}(w)\right\rangle=0$. Lastly, there are three remaining Majoranan fermions $\psi^{7,8,9}$ in the $s o(9)_{1}$ theory. They completely decouple with $\phi_{\sigma}$ and $\phi_{\rho}$. Although the vertex $e^{i \phi_{\rho}}$ anticommutes with $\psi^{7,8,9}$, this has no effect on any of our derivations. More importantly the "neutral" vertices $e^{i \phi_{\sigma}^{j}}$ commute with the remaining fermions.

In section 4.2.2.2, we defined the $\mathbb{Z}_{6}$ parafermion (4.104)

$$
\Psi=\frac{1}{\sqrt{3}}\left(e^{i \phi_{\sigma}^{1}} \psi^{7}+e^{i \phi_{\sigma}^{2}} \psi^{8}+e^{i \phi_{\sigma}^{3}} \psi^{9}\right)
$$


which is part of the $s o(3)_{3}^{-}$current (see (4.103)). It generates the rest of the $\mathbb{Z}_{6}$ parafermions

$$
\begin{aligned}
& \Psi^{2}= \frac{1}{\sqrt{15}}\left[\sum_{j=1}^{3} e^{i 2 \phi_{\sigma}^{j}}\right. \\
&\left.+2 i\left(e^{-i \phi_{\sigma}^{1}} \psi^{89}+e^{-i \phi_{\sigma}^{2}} \psi^{97}+e^{-i \phi_{\sigma}^{3}} \psi^{78}\right)\right] \\
& \Psi^{3}= \sqrt{\frac{2}{5}}\left[i \psi^{789}-\cos \left(\phi_{\sigma}^{1}-\phi_{\sigma}^{2}\right) \psi^{9}\right. \\
&\left.\quad-\cos \left(\phi_{\sigma}^{2}-\phi_{\sigma}^{3}\right) \psi^{7}-\cos \left(\phi_{\sigma}^{3}-\phi_{\sigma}^{1}\right) \psi^{8}\right] \\
& \Psi^{4}=\left(\Psi^{2}\right)^{\dagger}, \quad \Psi^{5}=\left(\Psi_{1}\right)^{\dagger}, \quad \Psi^{0}=\Psi^{6}=1
\end{aligned}
$$

where $\psi^{a b}=\psi^{a} \psi^{b}$ and $\psi^{a b c}=\psi^{a} \psi^{b} \psi^{c}$. Their conformal dimensions

$$
h_{\Psi^{m}}=\frac{m(6-m)}{6}
$$

as well as the fusion rules

$$
\begin{aligned}
\Psi^{m}(z) \Psi^{m^{\prime}}(w)= & \frac{c^{m m^{\prime}}}{(z-w)^{m m^{\prime} / 3}} \Psi^{m+m^{\prime}}(w)+\ldots \\
\Psi^{m}(z) \Psi^{6-m}(w)= & \frac{1}{(z-w)^{2 h_{\Psi^{m}}}} \\
& \times\left[1+\frac{2 h_{\Psi^{m}}}{c_{\mathbb{Z}_{6}}}(z-w)^{2} T_{\mathbb{Z}_{6}}+\ldots\right]
\end{aligned}
$$

match with the known result by Zamolodchikov and Fateev[144], for $T_{\mathbb{Z}_{6}}$ the energymomentum tensor (4.102) with central charge $c_{\mathbb{Z}_{6}}=5 / 4$ and

$$
c^{m m^{\prime}}=\sqrt{\frac{\left(m+m^{\prime}\right) !(6-m) !\left(6-m^{\prime}\right) !}{m ! m^{\prime} !\left(6-m-m^{\prime}\right) ! 6 !}} .
$$




\section{Appendix E}

\section{The $S$-matrices of the $G_{N}$ state}

The surface topological orders of the time reversal symmetric gapped coupled wire model are described in section 4.3. There are thirty two distinct topological states defined in eq.(4.123) and (4.126), which we repeat here.

$$
G_{N}= \begin{cases}S O(r)_{1}, & \text { for } N=2 r \\ S O(3)_{3} \bigotimes_{b} S O(r)_{1}, & \text { for } N=9+2 r\end{cases}
$$

In this appendix we summarize the modular properties of these states. In particular we present there braiding $S$-matrices (4.118)

$$
\mathcal{S}_{\mathrm{ab}}=\frac{1}{\mathcal{D}} \sum_{\mathbf{c}} d_{\mathbf{c}} N_{\mathrm{ab}}^{\mathbf{c}} \frac{\theta_{\mathbf{c}}}{\theta_{\mathbf{a}} \theta_{\mathbf{b}}}
$$

which are identical to the modular $S$-matrix[26] of the $\mathcal{G}_{N}$ WZW CFT. The fusion matrices $N_{\mathbf{a b}}^{\mathbf{c}}$ that characterize fusion rules $\mathbf{a} \times \mathbf{b}=\sum_{\mathbf{c}} N_{\mathbf{a b}}^{\mathbf{c}} \mathbf{c}$ can in turned be determined by $S$-matrix through the Verlinde formula[123] (4.87)

$$
N_{s_{1} s_{2}}^{s}=\sum_{s^{\prime}} \frac{\mathcal{S}_{s_{1} s^{\prime}} \mathcal{S}_{s_{2} s^{\prime}} \mathcal{S}_{s s^{\prime}}}{\mathcal{S}_{0 s^{\prime}}}
$$


The $G_{N}$ state is Abelian and carries four anyon types $1, \psi, s_{+}, s_{-}$when $N$ is a multiple of four. It is non-Abelian otherwise and carries three anyon types $1, \psi, \sigma$ when $N$ is $2 \bmod 4$, or seven anyon types $1, \alpha_{+} \gamma_{+}, \beta, \gamma_{-}, \alpha_{-}, f$ when $N$ is odd. The quasiparticle exchange statistics $\theta_{\mathbf{x}}$ and quantum dimensions $d_{\mathbf{x}}$ are summarized in table 4.2 and 4.3. The total quantum dimensions $\mathcal{D}=\sqrt{\sum_{\mathbf{x}} d_{\mathbf{x}}^{2}}$ are given by

$$
\mathcal{D}_{G_{N}}= \begin{cases}2 & \text { for } N \text { even } \\ 2 \csc (\pi / 8) & \text { for } N \text { odd }\end{cases}
$$

where $\csc (\pi / 8)=\sqrt{4+2 \sqrt{2}}$.

The $S$-matrices of $G_{N}$ for $N=2 r$ even are well-known and are given by those of the $S O(r)_{1}$ states. $[60,59]$

$$
\begin{aligned}
\mathcal{S}_{G_{N}}=\frac{1}{\mathcal{D}_{G_{N}}}\left(\begin{array}{cccc}
1 & 1 & 1 & 1 \\
1 & 1 & -1 & -1 \\
1 & -1 & i^{n} & -i^{n} \\
1 & -1 & -i^{n} & i^{n}
\end{array}\right), & \text { for } N=4 n, \\
\mathcal{S}_{G_{N}}=\frac{1}{\mathcal{D}_{G_{N}}}\left(\begin{array}{ccc}
1 & 1 & \sqrt{2} \\
1 & 1 & -\sqrt{2} \\
\sqrt{2} & -\sqrt{2} & 0
\end{array}\right), & \text { for } N=4 n+2 .
\end{aligned}
$$

The $S$-matrices for the odd $N$ cases are modification of the $G_{9}=S O(3)_{3}$ prototype $(4.88)$

$$
\mathcal{S}_{s_{1} s_{2}}^{S O(3)_{3}}=\frac{1}{2} \sin \left[\frac{\pi\left(2 s_{1}+1\right)\left(2 s_{2}+1\right)}{8}\right]
$$

where $s_{j}=0,1 / 2,1,3 / 2,2,5 / 2,3$ label the seven anyon types $1, \alpha_{+}, \gamma_{+}, \beta, \gamma_{-}, \alpha_{-}, f$ (see table 4.1). For $N=9+2 r \bmod 32$, the $S$-matrix of $G_{N}$ is given by

$$
\mathcal{S}_{G_{N}}=\mathcal{F}^{r} S^{e}(\lceil r / 2\rceil) \mathcal{F}^{-r}
$$


where $\lceil r / 2\rceil \geq r / 2$ is the smallest integral ceiling of $r / 2, \mathcal{S}^{e}(n)$ is the $S$-matrix when $r=2 n$ is even

$$
\mathcal{S}^{e}(n)_{s_{1} s_{2}}=i^{n\left(4 s_{1} s_{2}\right)^{2}} \mathcal{S}_{s_{1} s_{2}}^{S O(3)_{3}}
$$

and $\mathcal{F}$ is the operator that flips the fermion parity of $\alpha_{+} \leftrightarrow \alpha_{-}$and $\gamma_{+} \leftrightarrow \gamma_{-}$

$$
\mathcal{F}=\left(\begin{array}{lllll}
1 & & & & \\
& & & 1 & \\
& & 1 & 1 & \\
& & 1 & & \\
& & & & 1
\end{array}\right)
$$




\section{Bibliography}

[1] Ian Affleck, Tom Kennedy, Elliott H. Lieb, and Hal Tasaki. Rigorous results on valence-bond ground states in antiferromagnets. Phys. Rev. Lett., 59:799-802, Aug 1987.

[2] Ian Affleck, Tom Kennedy, Elliott H. Lieb, and Hal Tasaki. Valence bond ground states in isotropic quantum antiferromagnets. Comm. Math. Phys., 115(3):477-528, 1988.

[3] Ian Affleck and Andreas W. W. Ludwig. Universal noninteger "ground-state degeneracy" in critical quantum systems. Phys. Rev. Lett., 67:161-164, Jul 1991.

[4] F. Alet, S. Capponi, H. Nonne, P. Lecheminant, and I. P. McCulloch. Quantum criticality in the so(5) bilinear-biquadratic heisenberg chain. Phys. Rev. B, 83:060407, Feb 2011.

[5] Alexander Altland and Martin R. Zirnbauer. Nonstandard symmetry classes in mesoscopic normal-superconducting hybrid structures. Phys. Rev. B, 55:11421161, Jan 1997.

[6] Luigi Amico, Rosario Fazio, Andreas Osterloh, and Vlatko Vedral. Entanglement in many-body systems. Rev. Mod. Phys., 80:517-576, May 2008.

[7] Y. Aoki, Y. Wada, M. Saitoh, R. Nomura, Y. Okuda, Y. Nagato, M. Yamamoto, S. Higashitani, and K. Nagai. Observation of surface andreev bound states of superfluid ${ }^{3} \mathrm{He}$ by transverse acoustic impedance measurements. Phys. Rev. Lett., 95:075301, Aug 2005.

[8] Itai Arad, Alexei Kitaev, Zeph Landau, and Umesh Vazirani. An area law and sub-exponential algorithm for 1d systems. arXiv preprint arXiv:1301.1162, 2013.

[9] F. A. Bais and J. K. Slingerland. Condensate-induced transitions between topologically ordered phases. Phys. Rev. B, 79:045316, Jan 2009.

[10] F. Alexander Bais and Peter G. Bouwknegt. A classification of subgroup truncations of the bosonic string. Nuclear Physics B, 279:561 - 570, 1987. 
[11] F.A. Bais, F. Englert, A. Taormina, and P. Zizzi. Torus compactification for non-simply laced groups. Nuclear Physics B, 279:529 - 547, 1987.

[12] L Barbiero, L Dell'Anna, A Trombettoni, and VE Korepin. Haldane topological orders in motzkin spin chains. arXiv preprint arXiv:1701.05878, 2017.

[13] Luca Bombelli, Rabinder K. Koul, Joohan Lee, and Rafael D. Sorkin. Quantum source of entropy for black holes. Phys. Rev. D, 34:373-383, Jul 1986.

[14] P. Bonderson, C. Nayak, and X.-L. Qi. A time-reversal invariant topological phase at the surface of a 3D topological insulator. J. Stat. Mech., 9:16, September 2013 .

[15] Sergey Bravyi, Libor Caha, Ramis Movassagh, Daniel Nagaj, and Peter W Shor. Criticality without frustration for quantum spin-1 chains. Physical review letters, 109(20):207202, 2012.

[16] Pasquale Calabrese and John Cardy. Entanglement entropy and quantum field theory. Journal of Statistical Mechanics: Theory and Experiment, 2004(06):P06002, 2004.

[17] Pasquale Calabrese and John Cardy. Entanglement entropy and conformal field theory. Journal of Physics A: Mathematical and Theoretical, 42(50):504005, 2009 .

[18] Andrea Cappelli, Marina Huerta, and Guillermo R. Zemba. Thermal transport in chiral conformal theories and hierarchical quantum hall states. Nuclear Physics B, 636(3):568 - 582, 2002.

[19] John Cardy. Scaling and Renormalization in Statistical Physics. Cambridge University Press, 1996.

[20] X. Chen, L. Fidkowski, and A. Vishwanath. Symmetry enforced non-Abelian topological order at the surface of a topological insulator. Phys. Rev. B, 89:165132, April 2014.

[21] Xie Chen, Zheng-Cheng Gu, and Xiao-Gang Wen. Classification of gapped symmetric phases in one-dimensional spin systems. Phys. Rev. B, 83:035107, Jan 2011.

[22] Ching-Kai Chiu, Jeffrey C. Y. Teo, Andreas P. Schnyder, and Shinsei Ryu. Classification of topological quantum matter with symmetries. Rev. Mod. Phys., 88:035005, Aug 2016.

[23] H. Choi, J. P. Davis, J. Pollanen, and W. P. Halperin. Surface specific heat of ${ }^{3}$ He and andreev bound states. Phys. Rev. Lett., 96:125301, Mar 2006.

[24] L Dell'Anna, O Salberger, L Barbiero, A Trombettoni, and VE Korepin. Violation of cluster decomposition and absence of light cones in local integer and half-integer spin chains. Physical Review B, 94(15):155140, 2016. 
[25] Eric Dennis, Alexei Kitaev, Andrew Landahl, and John Preskill. Topological quantum memory. Journal of Mathematical Physics, 43(9):4452-4505, 2002.

[26] P. Di Francesco, P. Mathieu, and D. Senechal. Conformal Field Theory. Springer, 1999.

[27] Jens Eisert, Marcus Cramer, and Martin B Plenio. Colloquium: Area laws for the entanglement entropy. Reviews of Modern Physics, 82(1):277, 2010.

[28] V. J. Emery, E. Fradkin, S. A. Kivelson, and T. C. Lubensky. Quantum theory of the smectic metal state in stripe phases. Phys. Rev. Lett., 85:2160-2163, Sep 2000 .

[29] V.A. Fateev and A.B. Zamolodchikov. Self-dual solutions of the star-triangle relations in zn-models. Physics Letters A, 92(1):37 - 39, 1982.

[30] Paul Fendley, Matthew P. A. Fisher, and Chetan Nayak. Topological entanglement entropy from the holographic partition function. Journal of Statistical Physics, 126(6):1111-1144, 2007.

[31] Paul Fendley and Hubert Saleur. Bps kinks in the gross-neveu model. Phys. Rev. D, 65:025001, Nov 2001.

[32] Lukasz Fidkowski, Xie Chen, and Ashvin Vishwanath. Non-abelian topological order on the surface of a $3 \mathrm{~d}$ topological superconductor from an exactly solved model. Phys. Rev. X, 3:041016, Nov 2013.

[33] Lukasz Fidkowski and Alexei Kitaev. Effects of interactions on the topological classification of free fermion systems. Phys. Rev. B, 81:134509, Apr 2010.

[34] Lukasz Fidkowski and Alexei Kitaev. Topological phases of fermions in one dimension. Phys. Rev. B, 83:075103, Feb 2011.

[35] SK Foong and S Kanno. Proof of page's conjecture on the average entropy of a subsystem. Physical review letters, 72(8):1148, 1994.

[36] Eduardo Fradkin. Field Theories of Condensed Matter Physics. Cambridge University Press, second edition, 2013.

[37] Liang Fu and Erez Berg. Odd-parity topological superconductors: Theory and application to cuxbi2se3. Phys. Rev. Lett., 105:097001, Aug 2010.

[38] Liang Fu, C. L. Kane, and E. J. Mele. Topological insulators in three dimensions. Phys. Rev. Lett., 98:106803, 2007.

[39] D. Gioev and I. Klich. Entanglement entropy of fermions in any dimension and the Widom conjecture. Phys. Rev. Lett., 96(10):100503, 2006. 
[40] P. Goddard, W. Nahm, and D. Olive. Symmetric spaces, sugawara's energy momentum tensor in two dimensions and free fermions. Phys. Lett. B, 160(13):111 - 116, 1985.

[41] Gregory Gorohovsky, Rodrigo G. Pereira, and Eran Sela. Chiral spin liquids in arrays of spin chains. Phys. Rev. B, 91:245139, Jun 2015.

[42] Daniel Gottesman and MB Hastings. Entanglement versus gap for onedimensional spin systems. New journal of physics, 12(2):025002, 2010.

[43] David J. Gross and André Neveu. Dynamical symmetry breaking in asymptotically free field theories. Phys. Rev. D, 10:3235-3253, Nov 1974.

[44] F. D. M. Haldane. Continuum dynamics of the 1-d heisenberg antiferromagnet: Identification with the o(3) nonlinear sigma model. Physics Letters A, 93(9):464 - 468, 1983.

[45] F. D. M. Haldane. Nonlinear field theory of large-spin heisenberg antiferromagnets: Semiclassically quantized solitons of the one-dimensional easy-axis néel state. Phys. Rev. Lett., 50:1153-1156, Apr 1983.

[46] F. D. M. Haldane. Stability of chiral luttinger liquids and abelian quantum hall states. Phys. Rev. Lett., 74:2090-2093, Mar 1995.

[47] M. Z. Hasan and C. L. Kane. Topological insulators. Rev. Mod. Phys., 82:3045, 2010 .

[48] Matthew B Hastings. An area law for one-dimensional quantum systems. Journal of Statistical Mechanics: Theory and Experiment, 2007(08):P08024, 2007.

[49] C. Holzhey, F. Larsen, and F. Wilczek. High Energy Physics-Theory Title: Geometric and Renormalized Entropy in Conformal Field Theory. Journal reference: Nucl. Phys. B424, 443:467, 1994.

[50] Christoph Holzhey, Finn Larsen, and Frank Wilczek. Geometric and renormalized entropy in conformal field theory. Nuclear Physics B, 424(3):443 - 467, 1994.

[51] Sandy Irani. Ground state entanglement in one-dimensional translationally invariant quantum systems. Journal of Mathematical Physics, 51(2):022101, 2010 .

[52] R. Jackiw and C. Rebbi. Solitons with fermion number 1/2. Phys. Rev. D, 13:3398-3409, Jun 1976.

[53] B.-Q. Jin and V. E. Korepin. Quantum spin chain, toeplitz determinants and the fisher-hartwig conjecture. Journal of Statistical Physics, 116(1):79-95, 2004 . 
[54] C. L. Kane and Matthew P. A. Fisher. Quantized thermal transport in the fractional quantum hall effect. Phys. Rev. B, 55:15832-15837, Jun 1997.

[55] C. L. Kane, Ranjan Mukhopadhyay, and T. C. Lubensky. Fractional quantum hall effect in an array of quantum wires. Phys. Rev. Lett., 88:036401, Jan 2002.

[56] A. Kapustin, R. Thorngren, A. Turzillo, and Z. Wang. Fermionic Symmetry Protected Topological Phases and Cobordisms. ArXiv e-prints, June 2014.

[57] Fujikawa Kazuo and Hiroshi Suzuki. Path integrals and quantum anomalies. Oxford University Press, 2004.

[58] Mayukh Nilay Khan, Jeffrey C. Y. Teo, and Taylor L. Hughes. to appear soon.

[59] Mayukh Nilay Khan, Jeffrey C. Y. Teo, and Taylor L. Hughes. Anyonic symmetries and topological defects in abelian topological phases: An application to the ade classification. Phys. Rev. B, 90:235149, Dec 2014.

[60] A. Kitaev. Anyons in an exactly solved model and beyond. Ann. Phys., 321:2, 2006.

[61] A. Yu Kitaev. Unpaired majorana fermions in quantum wires. Phys.-Usp., 44:131, 2001.

[62] Alexei Kitaev. Periodic table for topological insulators and superconductors. AIP Conf. Proc., 1134:22, 2008.

[63] Alexei Kitaev and John Preskill. Topological entanglement entropy. Phys. Rev. Lett., 96:110404, 2006.

[64] Jelena Klinovaja and Daniel Loss. Integer and fractional quantum hall effect in a strip of stripes. The European Physical Journal B, 87:171, 2014.

[65] Jelena Klinovaja and Yaroslav Tserkovnyak. Quantum spin hall effect in strip of stripes model. Phys. Rev. B, 90:115426, Sep 2014.

[66] Nicolas Laflorencie. Quantum entanglement in condensed matter systems. Physics Reports, 646:1 - 59, 2016. Quantum entanglement in condensed matter systems.

[67] H. Blaine Lawson and Marie-Louise Michelsohn. Spin Geometry. Princeton University Press, 1990.

[68] Lionel Levine and Ramis Movassagh. The gap of the area-weighted motzkin spin chain is exponentially small. arXiv preprint: 1611.03147, 2016.

[69] J. M. Luttinger. Theory of thermal transport coefficients. Phys. Rev., 135:A1505-A1514, Sep 1964. 
[70] Tobias Meng. Fractional topological phases in three-dimensional coupled-wire systems. Phys. Rev. B, 92:115152, Sep 2015.

[71] Tobias Meng, Titus Neupert, Martin Greiter, and Ronny Thomale. Coupledwire construction of chiral spin liquids. Phys. Rev. B, 91:241106, Jun 2015.

[72] Tobias Meng and Eran Sela. Time reversal symmetry broken fractional topological phases at zero magnetic field. Phys. Rev. B, 90:235425, Dec 2014.

[73] Tobias Meng, Peter Stano, Jelena Klinovaja, and Daniel Loss. Helical nuclear spin order in a strip of stripes in the quantum hall regime. The European Physical Journal B, 87:203, 2014.

[74] M. A. Metlitski, L. Fidkowski, X. Chen, and A. Vishwanath. Interaction effects on 3D topological superconductors: surface topological order from vortex condensation, the 16 fold way and fermionic Kramers doublets. ArXiv e-prints, June 2014.

[75] Max A. Metlitski, C. L. Kane, and Matthew P. A. Fisher. Symmetry-respecting topologically ordered surface phase of three-dimensional electron topological insulators. Phys. Rev. B, 92:125111, Sep 2015.

[76] Eli J. Mlawer, Stephen G. Naculich, Harold A. Riggs, and Howard J. Schnitzer. Group-level duality of $\{W Z W\}$ fusion coefficients and chern-simons link observables. Nuclear Physics B, 352(3):863 - 896, 1991.

[77] Roger S.K. Mong, David J. Clarke, Jason Alicea, Netanel H. Lindner, Paul Fendley, Chetan Nayak, Yuval Oreg, Ady Stern, Erez Berg, Kirill Shtengel, and Matthew P.A. Fisher. Universal topological quantum computation from a superconductor-abelian quantum hall heterostructure. Phys. Rev. X, 4:011036, Mar 2014.

[78] J. E. Moore and L. Balents. Topological invariants of time-reversal-invariant band structures. Phys. Rev. B, 75:121306(R), 2007.

[79] Ramis Movassagh, Edward Farhi, Jeffrey Goldstone, Daniel Nagaj, Tobias J Osborne, and Peter W Shor. Unfrustrated qudit chains and their ground states. Physical Review A, 82(1):012318, 2010.

[80] Ramis Movassagh and Peter W. Shor. Supercritical entanglement in local systems: Counterexample to the area law for quantum matter. Proceedings of the National Academy of Sciences, 113(47):13278-13282, 2016.

[81] David F. Mross, Andrew Essin, and Jason Alicea. Composite dirac liquids: Parent states for symmetric surface topological order. Phys. Rev. X, 5:011011, Feb 2015.

[82] Ranjan Mukhopadhyay, C. L. Kane, and T. C. Lubensky. Crossed sliding luttinger liquid phase. Phys. Rev. B, 63:081103, Feb 2001. 
[83] S. Murakawa, Y. Tamura, Y. Wada, M. Wasai, M. Saitoh, Y. Aoki, R. Nomura, Y. Okuda, Y. Nagato, M. Yamamoto, S. Higashitani, and K. Nagai. New anomaly in the transverse acoustic impedance of superfluid ${ }^{3} \mathrm{He}-\mathrm{B}$ with a wall coated by several layers of ${ }^{4}$ He. Phys. Rev. Lett., 103:155301, Oct 2009.

[84] S.G. Naculich, H.A. Riggs, and H.J. Schnitzer. Group-level duality in $\{W Z W\}$ models and chern-simons theory. Physics Letters B, 246:417 - 422, 1990.

[85] Chetan Nayak, Steven H. Simon, Ady Stern, Michael Freedman, and Sankar Das Sarma. Non-abelian anyons and topological quantum computation. Rev. Mod. Phys., 80:1083, 2008.

[86] Titus Neupert, Claudio Chamon, Christopher Mudry, and Ronny Thomale. Wire deconstructionism of two-dimensional topological phases. Phys. Rev. B, 90:205101, Nov 2014.

[87] H.B. Nielsen and Masao Ninomiya. The adler-bell-jackiw anomaly and weyl fermions in a crystal. Phys. Lett. B, 130(6):389 - 396, 1983.

[88] C. S. O'Hern, T. C. Lubensky, and J. Toner. Sliding phases in $X Y$ models, crystals, and cationic lipid-dna complexes. Phys. Rev. Lett., 83:2745-2748, Oct 1999.

[89] Yuval Oreg, Eran Sela, and Ady Stern. Fractional helical liquids in quantum wires. Phys. Rev. B, 89:115402, Mar 2014.

[90] Román Orús. A practical introduction to tensor networks: Matrix product states and projected entangled pair states. Annals of Physics, 349:117 - 158, October 2014.

[91] Don N Page. Average entropy of a subsystem. Physical review letters, 71(9):1291, 1993.

[92] David Poulin, Angie Qarry, Rolando Somma, and Frank Verstraete. Quantum simulation of time-dependent hamiltonians and the convenient illusion of hilbert space. Phys. Rev. Lett., 106:170501, Apr 2011.

[93] Xiao-Liang Qi, Taylor L. Hughes, S. Raghu, and Shou-Cheng Zhang. Timereversal-invariant topological superconductors and superfluids in two and three dimensions. Phys. Rev. Lett., 102:187001, May 2009.

[94] Xiao-Liang Qi, Taylor L. Hughes, and Shou-Cheng Zhang. Topological field theory of time-reversal invariant insulators. Phys. Rev. B, 78:195424, 2008.

[95] Xiao-Liang Qi, Edward Witten, and Shou-Cheng Zhang. Axion topological field theory of topological superconductors. Phys. Rev. B, 87:134519, Apr 2013. 
[96] Giovanni Ramírez, Javier Rodríguez-Laguna, and Germán Sierra. From conformal to volume law for the entanglement entropy in exponentially deformed critical spin 1/2 chains. Journal of Statistical Mechanics: Theory and Experiment, 2014(10):P10004, 2014.

[97] N. Read and Dmitry Green. Paired states of fermions in two dimensions with breaking of parity and time-reversal symmetries and the fractional quantum hall effect. Phys. Rev. B, 61:10267, 2000.

[98] N. Read and G. Moore. Nonabelions in the fractional quantum hall effect. Nucl. Phys. B, 360:362, 1991.

[99] N. Read and G. Moore. Fractional quantum hall effect and nonabelian statistics. Progress of Theoretical Physics Supplement, 107:157-166, 1992.

[100] Rahul Roy. Topological phases and the quantum spin hall effect in three dimensions. Phys. Rev. B, 79:195322, 2009.

[101] Eran Sagi and Yuval Oreg. Non-abelian topological insulators from an array of quantum wires. Phys. Rev. B, 90:201102, Nov 2014.

[102] Eran Sagi and Yuval Oreg. From an array of quantum wires to three-dimensional fractional topological insulators. Phys. Rev. B, 92:195137, Nov 2015.

[103] Eran Sagi, Yuval Oreg, Ady Stern, and Bertrand I. Halperin. Imprint of topological degeneracy in quasi-one-dimensional fractional quantum hall states. Phys. Rev. B, 91:245144, Jun 2015.

[104] Olof Salberger and Vladimir Korepin. Fredkin spin chain. arXiv preprint arXiv:1605.03842, 2016.

[105] Olof Salberger, Takuma Udagawa, Zhao Zhang, Hosho Katsura, Israel Klich, and Vladimir Korepin. Deformed fredkin spin chain with extensive entanglement. arXiv preprint arXiv:1611.04983, 2016.

[106] Raul A. Santos, Chia-Wei Huang, Yuval Gefen, and D. B. Gutman. Fractional topological insulators: From sliding luttinger liquids to chern-simons theory. Phys. Rev. B, 91:205141, May 2015.

[107] Satoshi Sasaki, M. Kriener, Kouji Segawa, Keiji Yada, Yukio Tanaka, Masatoshi Sato, and Yoichi Ando. Topological superconductivity in $\mathrm{cu}_{x} \mathrm{bi}_{2} \mathrm{se}_{3}$. Phys. Rev. Lett., 107:217001, Nov 2011.

[108] A. N. Schellekens and N. P. Warner. Conformal subalgebras of kac-moody algebras. Phys. Rev. D, 34:3092-3096, Nov 1986.

[109] Andreas P. Schnyder, Shinsei Ryu, Akira Furusaki, and Andreas W. W. Ludwig. Classification of topological insulators and superconductors in three spatial dimensions. Phys. Rev. B, 78:195125, 2008. 
[110] Siddhartha Sen. Average entropy of a quantum subsystem. Physical review letters, 77(1):1, 1996.

[111] T. Senthil. Symmetry Protected Topological phases of Quantum Matter. ArXiv e-prints, May 2014.

[112] Inbar Seroussi, Erez Berg, and Yuval Oreg. Topological superconducting phases of weakly coupled quantum wires. Phys. Rev. B, 89:104523, Mar 2014.

[113] R. Shankar and E. Witten. The s-matrix of the kinks of the $(\bar{g} \bar{\gamma} \psi)^{2}$ model. Nuclear Physics B, 141(4):349 - 363, 1978.

[114] S. L. Sondhi and Kun Yang. Sliding phases via magnetic fields. Phys. Rev. B, 63:054430, Jan 2001.

[115] E. M. Stoudenmire, David J. Clarke, Roger S. K. Mong, and Jason Alicea. Assembling fibonacci anyons from a $\digamma_{3}$ parafermion lattice model. Phys. Rev. B, 91:235112, Jun 2015 .

[116] Jeffrey C. Y. Teo and C. L. Kane. Topological defects and gapless modes in insulators and superconductors. Phys. Rev. B, 82:115120, Sep 2010.

[117] Jeffrey C. Y. Teo and C. L. Kane. From luttinger liquid to non-abelian quantum hall states. Phys. Rev. B, 89:085101, Feb 2014.

[118] Jeffrey C.Y. Teo, Taylor L. Hughes, and Eduardo Fradkin. Theory of twist liquids: Gauging an anyonic symmetry. Annals of Physics, 360(0):349 - 445, 2015.

[119] Hong-Hao Tu and Román Orús. Effective field theory for the $\mathrm{SO}(n)$ bilinearbiquadratic spin chain. Phys. Rev. Lett., 107:077204, Aug 2011.

[120] Hong-Hao Tu, Guang-Ming Zhang, and Tao Xiang. Class of exactly solvable so(n) symmetric spin chains with matrix product ground states. Phys. Rev. B, 78:094404, Sep 2008.

[121] Ari M. Turner, Frank Pollmann, and Erez Berg. Topological phases of onedimensional fermions: An entanglement point of view. Phys. Rev. B, 83:075102, Feb 2011.

[122] Takuma Udagawa and Hosho Katsura. Finite-size gap, magnetization, and entanglement of deformed fredkin spin chain. arXiv preprint arXiv:1701.00346, 2017.

[123] Erik Verlinde. Fusion rules and modular transformations in 2d conformal field theory. Nucl. Phys. B, 300:360, 1988.

[124] Ashvin Vishwanath and David Carpentier. Two-dimensional anisotropic nonfermi-liquid phase of coupled luttinger liquids. Phys. Rev. Lett., 86:676-679, Jan 2001. 
[125] G Vitagliano, A Riera, and JI Latorre. Volume-law scaling for the entanglement entropy in spin-1/2 chains. New Journal of Physics, 12(11):113049, 2010.

[126] G. E. Volovik. Quantum hall and chiral edge states in thin 3he-a film. JETP Letters, 55:368, 1992.

[127] G. E. Volovik. Universe in a helium droplet. Oxford University Press, Oxford UK, 052003.

[128] Chong Wang, Andrew C. Potter, and T. Senthil. Gapped symmetry preserving surface state for the electron topological insulator. Phys. Rev. B, 88:115137, Sep 2013.

[129] Chong Wang, Andrew C. Potter, and T. Senthil. Classification of interacting electronic topological insulators in three dimensions. Science, 343(6171):629$631,2014$.

[130] Chong Wang and T. Senthil. Interacting fermionic topological insulators/superconductors in three dimensions. Phys. Rev. B, 89:195124, May 2014.

[131] X. G. Wen. Vacuum degeneracy of chiral spin states in compactified space. Phys. Rev. B, 40:7387-7390, Oct 1989.

[132] Xiao-Gang Wen. Topological order in rigid states. Int. J. Mod. Phys. B, 04(02):239-271, 1990.

[133] Xiao-Gang Wen. Topological orders and edge excitations in fractional quantum hall states. Advances in Physics, 44(5):405, 1995.

[134] Xiao-Gang Wen. Quantum Field Theory of Many Body Systems. Oxford Univ. Press, Oxford, 2004.

[135] Xiao-Gang Wen and Qian Niu. Ground-state degeneracy of the fractional quantum hall states in the presence of a random potential and on high-genus riemann surfaces. Phys. Rev. B, 41:9377, 1990.

[136] J. Wess and B. Zumino. Consequences of anomalous ward identities. Physics Letters B, 37(1):95 - 97, 1971.

[137] Frank Wilczek. Fractional Statistics and Anyon Superconductivity. World Scientific, 1990.

[138] Edward Witten. Some properties of the $(\bar{\psi} \psi)^{2}$ model in two dimensions. Nuclear Physics B, 142(3):285 - 300, 1978.

[139] Edward Witten. Global aspects of current algebra. Nuclear Physics B, $223(2): 422-432,1983$.

[140] Edward Witten. Nonabelian bosonization in two dimensions. Comm. Math. Phys., 92(4):455-472, 1984. 
[141] Edward Witten. Fermion path integrals and topological phases. Rev. Mod. Phys., 88:035001, Jul 2016.

[142] M.M. Wolf. Violation of the entropic area law for fermions. Physical Review Letters, 96(1):10404, 2006.

[143] Di Xiao, Ming-Che Chang, and Qian Niu. Berry phase effects on electronic properties. Rev. Mod. Phys., 82:1959-2007, Jul 2010.

[144] A. B. Zamolodchikov and V. A. Fateev. Nonlocal (parafermion) currents in two-dimensional conformal quantum field theory and self-dual critical points in zn-symmetric statistical systems. Sov. Phys. JETP, 62:215, 1985.

[145] Alexandre B. Zamolodchikov and Alexey B. Zamolodchikov. Exact s matrix of gross-neveu âelementaryâ fermions. Physics Letters B, 72(4):481 - 483, 1978.

[146] Zhao Zhang, Amr Ahmadain, and Israel Klich. Novel quantum phase transition from bounded to extensive entanglement. Proceedings of the National Academy of Sciences, 114(19), 2017.

[147] Israel Klich Zhao Zhang. Entropy, gap and a multi-parameter deformation of the fredkin spin chain. ArXiv e-prints 1702.03581, 2017. 